\title{
AUTOMATED TUNING OF A PIEZOELECTRIC POWER HARVESTING CANTILEVER BEAM VIA THE APPLICATION OF AN AXIAL LOAD
}

\author{
by \\ Kenneth See Jin Tam \\ Bachelor of Engineering, Ryerson University, 2011
}

\author{
A thesis \\ presented to Ryerson University \\ in partial fulfillment of the \\ requirements for the degree of \\ Master of Applied Science \\ in the Program of
}

Mechanical and Industrial Engineering

Toronto, Ontario, Canada, 2013

CKenneth See Jin Tam 2013 


\section{AUTHOR'S DECLARATION FOR ELECTRONIC SUBMISSION OF A THESIS}

I hereby declare that I am the sole author of this thesis. This is a true copy of the thesis, including any required final revisions, as accepted by my examiners.

I authorize Ryerson University to lend this thesis to other institutions or individuals for the purpose of scholarly research.

I further authorize Ryerson University to reproduce this thesis by photocopying or by other means, in total or in part, at the request of other institutions or individuals for the purpose of scholarly research.

I understand that my thesis may be made electronically available to the public. 


\begin{abstract}
$\underline{\text { Abstract }}$
This thesis deals with automatic tuning of piezoelectric power harvesters. A prototype was constructed to verify the effect of the application of an axial load on a cantilever beam and the effectiveness of increasing the power harvested from a piezoelectric beam via axial loading. It was shown, experimentally, that the natural frequency of a piezoelectric beam harvester can be changed over a range of $25 \mathrm{~Hz}$. From the experimental results it was shown that the power can increase up to seven times when tuned in comparison to unturned. Computer simulations were used to demonstrate a closed loop tuning system's ability to apply an axial load effectively onto a piezoelectric cantilever beam in response to ambient vibrations. The closed loop tuning system is a viable option for tuning and can lead to increased power when applying the axial load suggested by the tuning system.
\end{abstract}




\section{$\underline{\text { Acknowledgements }}$}

"The LORD is my strength and my shield; my heart trusted in him, and I am helped: therefore my heart greatly rejoiceth; and with my song will I praise him.” Psalm 28:7

I would also like to thank the following people for their contribution to the success of the project:

Dr. Ahmad Ghasempoor and Dr. Siyuan He, my supervisors, for their advice and guidance throughout the course of the project.

Sheng Wei, for teaching me how to use the finite element program ANSYS.

Lester Tam, my brother, for his emotional support and his hard work in proofreading.

Fan Chao, Xue Yuan, Farzana Husain and Zewdu Hailu, my labmates, for their camaraderie during the course of my studies.

Alfred Tam and Katherine Tam, my parents, for their unwavering moral support. 


\section{Table of Contents}

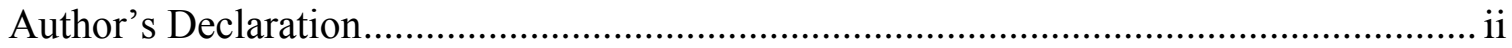

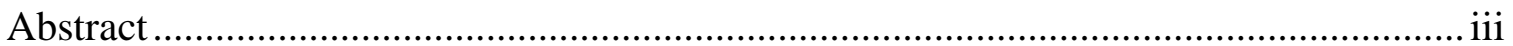

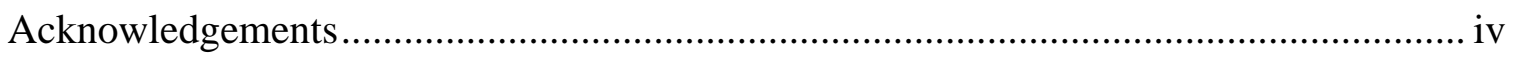

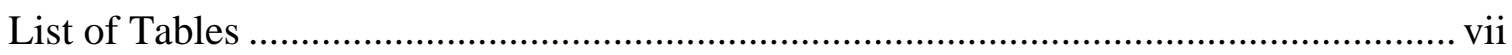

List of Figures ................................................................................................ vii

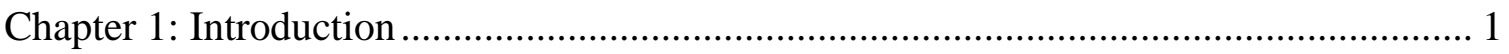

Chapter 2: Literature Review .............................................................................. 3

2.1 Principle of Operations of Power Harvesters ……....................................................... 3

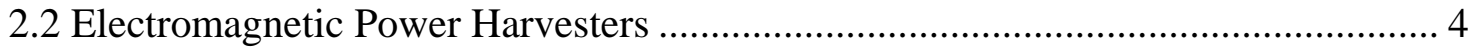

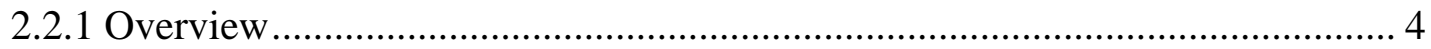

2.2.2 Existing Technologies/ Prototypes ............................................................... 4

2.3 Electrostatic Power Harvesters.......................................................................... 8

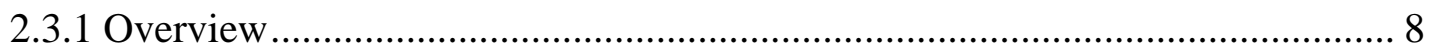

2.3.2 Existing Technologies/ Prototypes ................................................................. 13

2.4 Piezoelectric Power Harvesters ............................................................................... 18

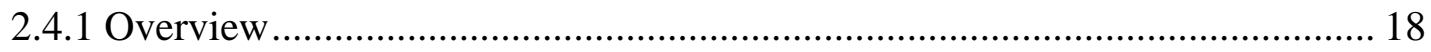

2.4.2 Existing Technologies/ Prototypes ................................................................. 20

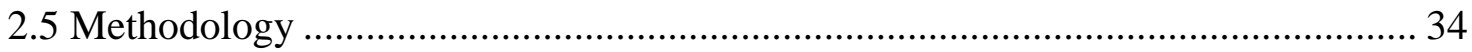

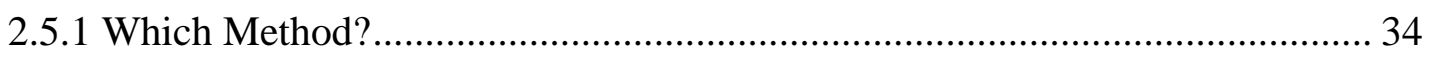

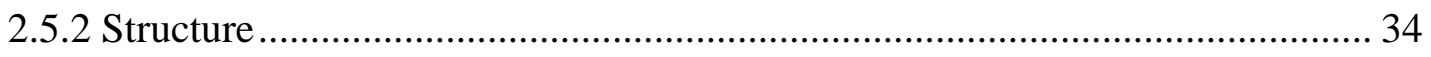

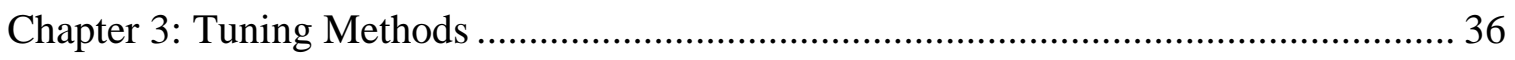

3.1 Tuning Approaches for Vibration-Powered Power Harvesters................................ 36

3.2 Effect of Axial Load on Natural Frequency of a Cantilever Beam.......................... 37

3.2.1 Results: Theoretical versus Simulation.......................................................... 37 


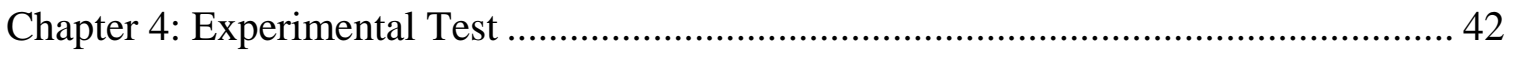

4.1 Power Harvesting Beam and Axial Load Adjustment Mechanism........................ 42

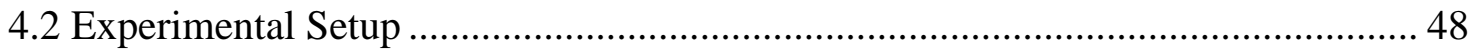

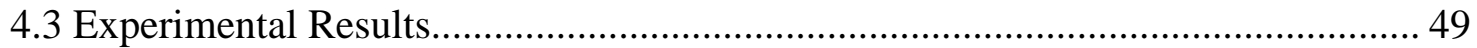

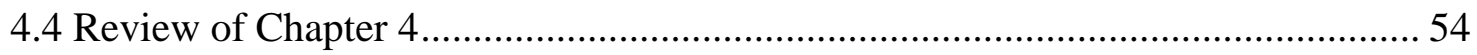

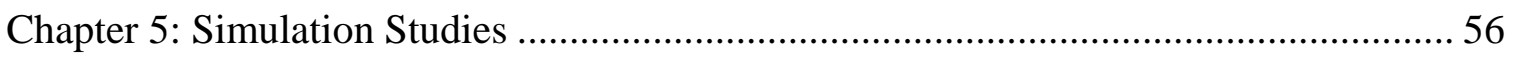

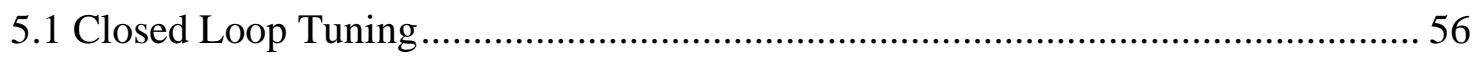

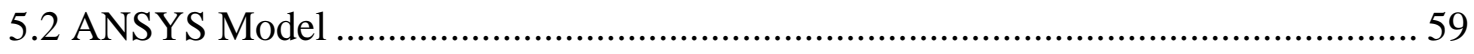

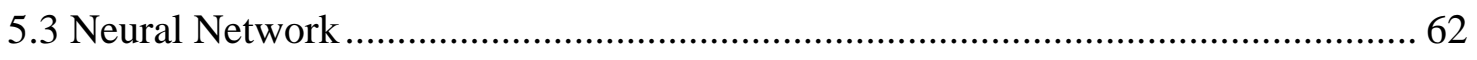

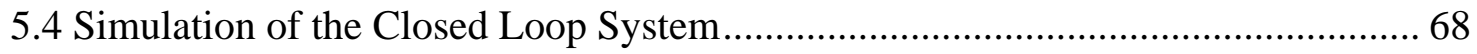

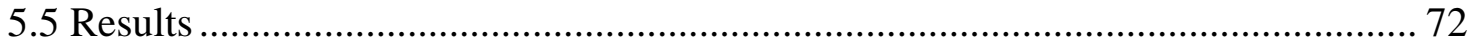

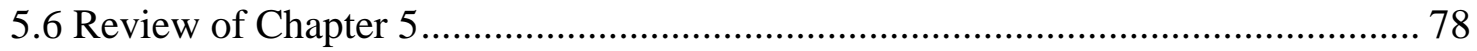

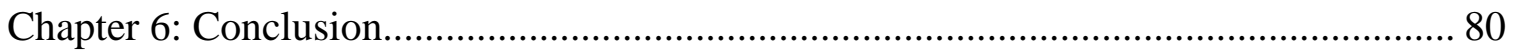

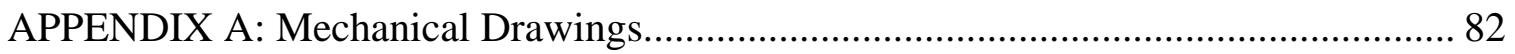

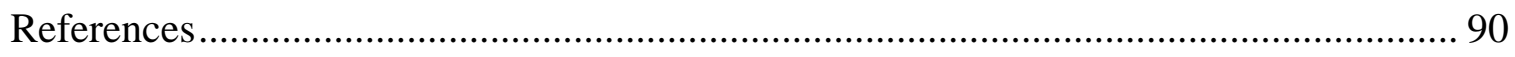




\section{List of Tables}

Table 1 - Results of Depesse's simulation [22] .......................................................... 15

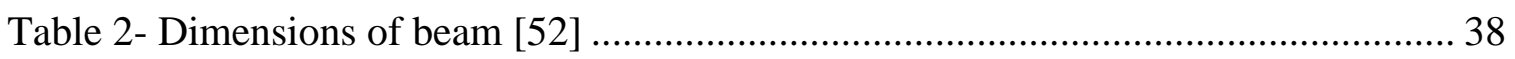

Table 3 - Power output of tensile mode .......................................................................... 52

Table 4- Power output of compression mode ……………………………………….... 52

Table 5- Table showing effectiveness of tuning ............................................................. 53

Table 6 - Acceleration and frequency of common vibration sources [2] ......................... 63

Table 7 - Power values of the untuned and tuned scenarios ............................................. 77

\section{List of Figures}

Figure 1 - Model of MEMS power harvester [4] ...................................................... 3

Figure 2-(a) rotational generator (b) oscillatory generator (c) hybrid generator [6]........... 4

Figure 3 - Diaphragm electromagnetic generator [7] ................................................. 5

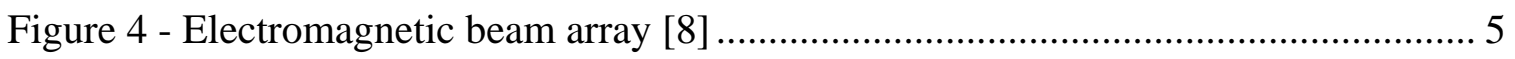

Figure 5 - Low frequency electromagnetic generator [10] ........................................... 6

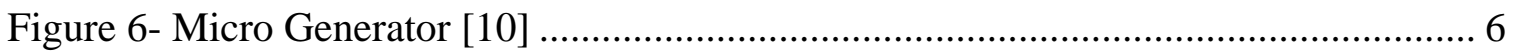

Figure 7- Wide bandwidth device design [12]......................................................... 7

Figure 8-Fabricated wide bandwidth device [12] ........................................................... 7

Figure 9- Electromagnetic harvester with stopper [13] ................................................. 8

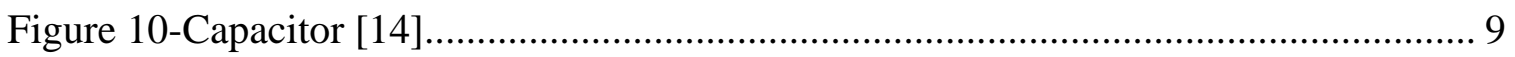

Figure 11 - Charge voltage plot with both charge and voltage constrained charging

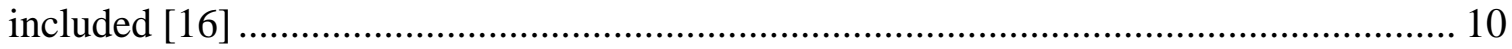

Figure 12- Circuit for a voltage constrained harvesters [18] ........................................ 11

Figure 13 - Logic circuit to activate the switches [18] ................................................... 11

Figure 14 - Charge constrained circuit [17] ............................................................. 12

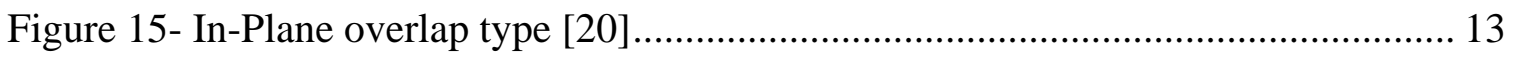

Figure 16- In-plane gap closing type [20]................................................................ 13

Figure 17- Out of plane gap closing type [20] ............................................................ 13

Figure 18 - First electrostatic harvester [17] ............................................................... 14

Figure 19 - Macro scale electrostatic harvester [22] .................................................. 15

Figure 20 - Innovative electrostatic harvester [23] ..................................................... 16 
Figure 21 -Fabricated electrostatic harvester [24] ........................................................ 16

Figure 22 - Electrostatic harvester [24] ................................................................ 17

Figure 23 - Novel electrostatic harvester [15] ........................................................... 17

Figure 24 - Rotary electrostatic harvester [25] ......................................................... 18

Figure 25 - Two modes of piezoelectric materials [2] ................................................... 19

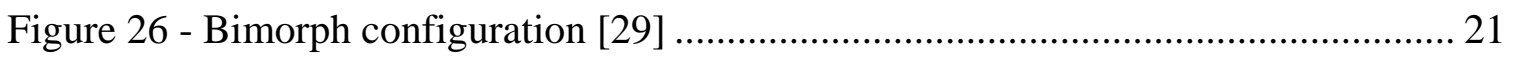

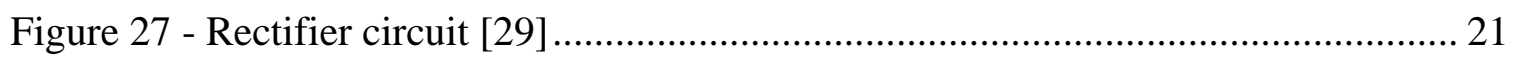

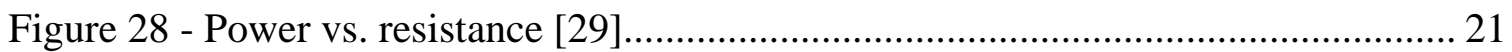

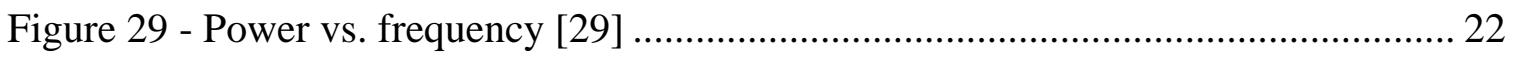

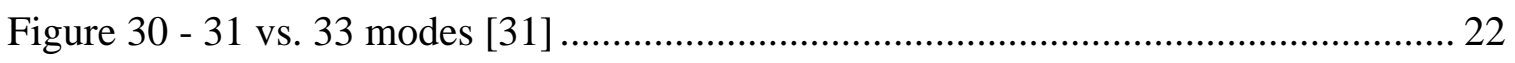

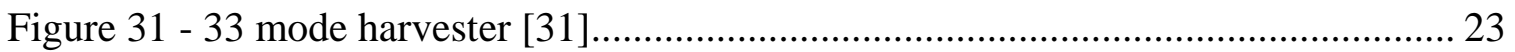

Figure 32 - SEM photo of interdigitated electrodes [31] ................................................ 24

Figure 33 - Piezoelectric harvester [32] .................................................................. 24

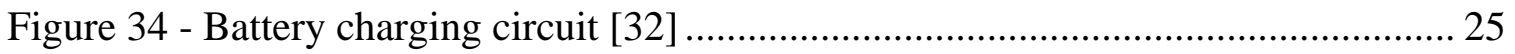

Figure 35 - Active tuning device [33] ..................................................................... 25

Figure 36 - Nickel proof mass [34] ......................................................................... 26

Figure 37 - SEM photo of device and nickel mass [35] ............................................. 26

Figure 38 - Magnet tuning harvester [36] ............................................................... 27

Figure 39 - Silicon mass cantilever beam [37] ......................................................... 27

Figure 40 - Another 33 mode [38] .......................................................................... 28

Figure 41 - 31 vs. 33 mode generators [39] ............................................................. 28

Figure 42 - Magnetic and piezoelectric hybrid harvester [40] ........................................ 29

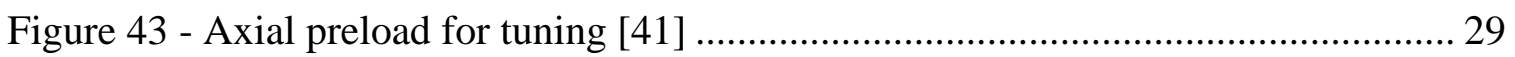

Figure 44 - Poling directions [42] .......................................................................... 30

Figure 45 - AlN harvester with stoppers [43] .......................................................... 30

Figure 46 - PZT cantilever design [44] ................................................................ 31

Figure 47 - Wideband harvester with stopper [45] ........................................................ 31

Figure 48 - Wide bandwidth harvester [46] ................................................................. 32

Figure 49 - Axial load tuning harvester [47] ............................................................ 33

Figure 50 - Bending torsion harvester[48]................................................................ 33

Figure 51 - Magnetic axial tuning bimorph [49] ............................................................. 34 
Figure 52- Layout of 427.0085.11Z beam [52] ………………………………..... 38

Figure 53 - Axial load vs. frequency graph based on theoretical equations ..................... 39

Figure 54 - Harmonic sweep of beam without tip mass ANSYS results........................... 40

Figure 55 - Cantilever beam with tip mass ...................................................................... 40

Figure 56 - Harmonic sweep of beam with tip mass ANSYS results ................................ 41

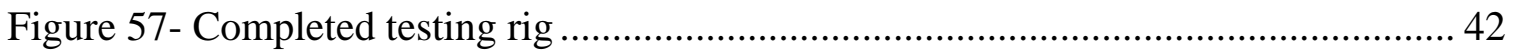

Figure 58 - Piezoelectric beam .................................................................................. 43

Figure 59- Relation between magnetic force and distance when one magnet is placed on

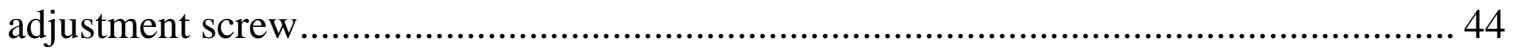

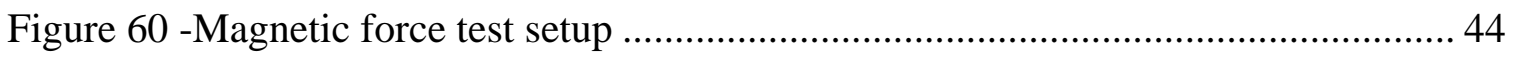

Figure 61 -Relation between magnetic force and distance when two magnets are placed on adjustment screw................................................................................................ 45

Figure 62 -Two magnets on adjustment screw ……………………………………..... 45

Figure 63 -Relation between magnetic force and distance when three magnets are placed

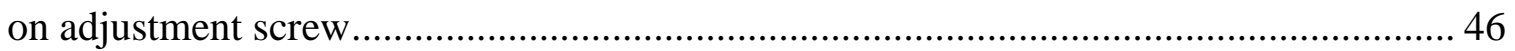

Figure 64 - Three magnets on adjustment screw ..................................................... 46

Figure 65 - Rendering of experimental setup for shaker .............................................. 47

Figure 66 -Electric circuit of experimental setup ………………………………….... 47

Figure 67 - Schematic of experimental setup connections ............................................. 48

Figure 68 - Experimental setup................................................................................ 49

Figure 69 - Relation between the natural frequency and the tension preload................... 50

Figure 70 - Relation between the natural frequency and the compression preload .......... 50

Figure 71- Relation between the natural frequency and both compression and tension preload............................................................................................................... 51

Figure 72- Comparison between tuned and untuned cases .......................................... 53

Figure 73- Relationship between average power and excitation frequencies of various tension axial load cases .............................................................................................. 54

Figure 74-Relationship between average power and excitation frequencies of various

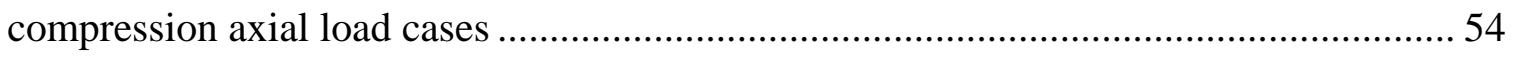

Figure 75 - Diagram of an axial load tuning system..................................................... 57

Figure 76 - Diagram of axial load tuning system simulation........................................... 57 
Figure 77 - Flow chart for closed loop system

Figure 78 - Mesh sensitivity analysis to help determine the minimum amount of elements

Figure 79 - Screenshot of the model in ANSYS

Figure 80 - Number of hidden neurons vs. error plot to help determine the ideal number of hidden neurons.

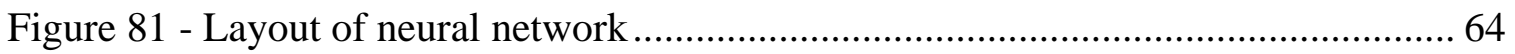

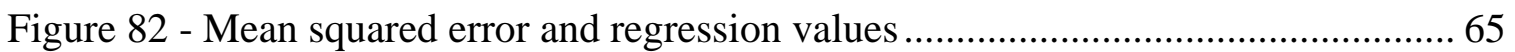

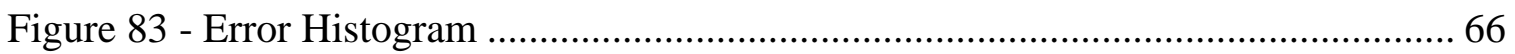

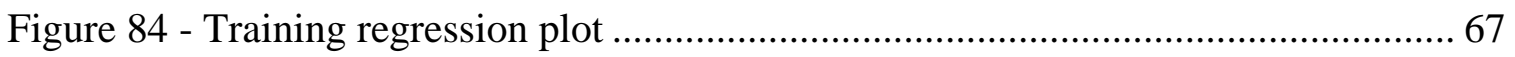

Figure 85 - MSE vs. Epochs for best performance ..................................................... 68

Figure 86 - Screenshot from Simulink model of the closed loop system ...................... 71

Figure 87 - Power vs. Resistance to find the optimal resistor value ............................. 72

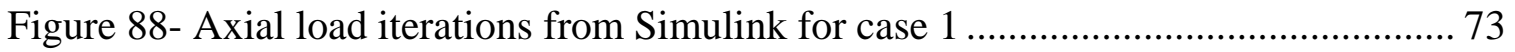

Figure 89 - Axial load iterations from Simulink for case 2 ..................................... 74

Figure 90 - Axial load iterations from Simulink for case 3 .................................... 75

Figure 91- Axial load iterations from Simulink for case 4 ..................................... 76

Figure 92 - Comparison of the power harvested from untuned and tuned scenarios of each case. 


\section{Chapter 1: Introduction}

Humans have been using energy from the environment for centuries, from ancient windmills to modern-day solar panels. In nearly all cases, energy was converted or harnessed to produce useful work. One area of recent development is in the realm of Micro Electrical Mechanical Systems (MEMS). MEMS power harvesting, also known as energy scavenging is the conversion of energy in the environment into electrical energy using microscale devices. Devices on the microscale level have dimensions between one millimetre and one micron. Examples of energy sources for energy scavenging include: solar, temperature gradients, vibrations and electromagnetic fields [1]. In particular, vibrations warrant further research due to their widespread nature and the ability to produce a reasonable amount of power for the size of the device $\left(250 \mu \mathrm{W} / \mathrm{cm}^{3}\right)[2]$.

Research in this area is largely directed towards reducing the size of power sources for electronic devices. Additionally this technology may lead to augmented battery life or in some cases, replace batteries all together. Many researchers believe that this technology would be suitable for powering wireless sensors or embedded sensors. Since these sensors are often placed in hard to access locations, attaching a power harvesting device would reduce the cost of maintenance and battery replacement. Also not having to run wires to the sensors will decrease the cost and complexity during installation of the sensor. In addition to wireless sensors, other applications include biomedical implants, monitoring devices and other small electronic devices [1]. The applications are promising and as such much has been invested into researching this technology. The ultimate goal is to create a harvester that can efficiently and effectively convert vibrations energy into usable electricity.

In this thesis, ambient vibrations will be investigated as the form of environmental energy. Since ambient vibrations are ubiquitous, they are an attractive choice for harvesting.

There are numerous ways to harvest vibrations and different methods to improve on the current technology to increase harvesting power. It is the aim of this thesis to investigate and examine the previous scholarship in the area of power harvesting and further explore new methods using piezoelectric devices. 
The success of vibration energy harvesting hinges on the ability to match the environmental frequency of excitation with the energy harvester's natural frequency; matching frequencies exploits the resonance phenomenon which allows the production of greater amounts of power. The ability to match these frequencies is the major hurdle facing power harvesters today.

This thesis will investigate the tuning of the energy harvester's natural frequency. A popular design of a piezoelectric energy harvester is a cantilever beam. If an axial load is applied to the cantilever beam the natural frequency can be changed this can be regarded as a form of tuning. The objective of this thesis is to examine the application of a closed loop tuning system for tuning the beam. Also to build a physical prototype to discover how much additional power can be harvested from tuning.

Chapter 2 is a literature review of previous research in the field of power harvesting. Chapter 3 summarizes the tuning approaches and establishes the theoretical basis for adaptable tuning. Chapter 4 presents the results of experimental verification of the effect of axial load in natural frequency of the harvester. Chapter 5 focuses on the development and results of a closed loop tuning system for automated tuning of the harvester. The discussions are contained in Chapter 6 and finally the findings of the thesis are summarized and further work is discussed in Chapter 7. 


\section{Chapter 2: Literature Review}

\subsection{Principle of Operations of Power Harvesters}

The initial idea and model for a MEMS based vibration harvester was proposed in a conference in 1995 by C.B. Williams and R. B. Yates [3]. The model, shown in Figure 1, consisted of a mass attached to a frame via a spring and a damper. The frame was excited by ambient motion or vibrations. When the frame is accelerated, the mass inside the frame moved relative to the frame causing displacement. Work was therefore performed on the damper by the mass as it came into contact [4]. The damper in this initial model represents a transducer, which is a device that converts vibrations in to electricity. Often devices have a small range of frequency in which they produce the greatest electricity (low bandwidth). One approach of researchers and designers is to optimize the design such that its natural frequency matches the frequency to which their device is being excited. The other approach is to widen the range of frequencies (bandwidth) one's device is able to effectively harvest from. Most recently researchers are looking into methods to have the devices adjust their natural frequency and in a sense tune themselves to match the frequency of the excitation. Many sources of vibration found in modern urban settings have low frequencies that are usually less than $100 \mathrm{~Hz}$ [2].

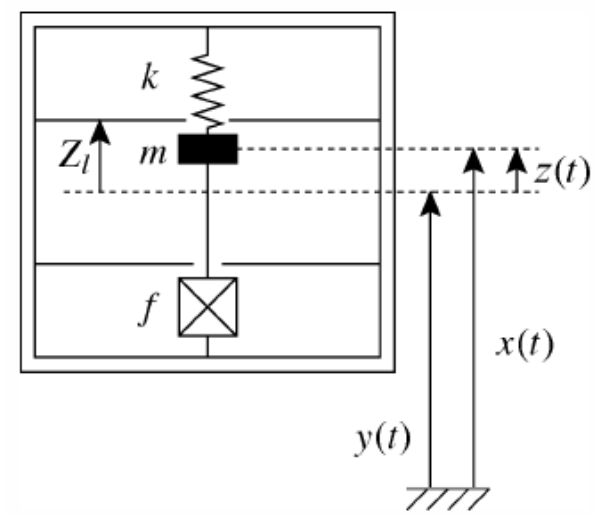

Figure 1 - Model of MEMS power harvester [4]

Currently there are three main types of transducers being used for MEMS power harvesting. The types are: electromagnetic, electrostatic and piezoelectric. Electromagnetic transducers are based on the principle of electromagnetic induction and electrostatic ones are based on a variable capacitors and changing the capacitance. On the other hand, piezoelectric transducers use piezoelectric materials, which generate a voltage when mechanical stress or strain is applied. 


\subsection{Electromagnetic Power Harvesters}

\subsubsection{Overview}

Electromagnetic transducers are based on the principle of electromagnetic induction which tells us that an electromotive force is generated in a conductor when the magnetic flux passing through the conductor changes. This means moving a conductor through a magnetic field causes an electric current in the conductor. The form that this usually takes is a permanent magnet and a coil moving relative to each other. The number of coils, strength of the magnetic field and velocity of the motion will affect the amount of electricity generated [5].

In the Figure 2 are typical generators using permanent magnets. The rotational generator uses rotational motion with the magnetic rotor spinning while the coils are held stationary. This generator can be attached to turbines or heat engines to generate electricity. The oscillatory generator is a mass spring system with the magnet as the mass and fixed coils. As the magnet moves, an electromotive force is produced in the coils. This generator captures vibrations and its maximum power is produce when it is vibrated at the resonant frequency of the generator in Figure $2 \mathrm{~b}$. The hybrid generator captures vibrations and turns it into rotational motion; this design overcomes the restriction of the oscillatory generator which is only effective in a small range of frequencies [6]. Below Figure 2 depicts these three types of electromagnetic generators.
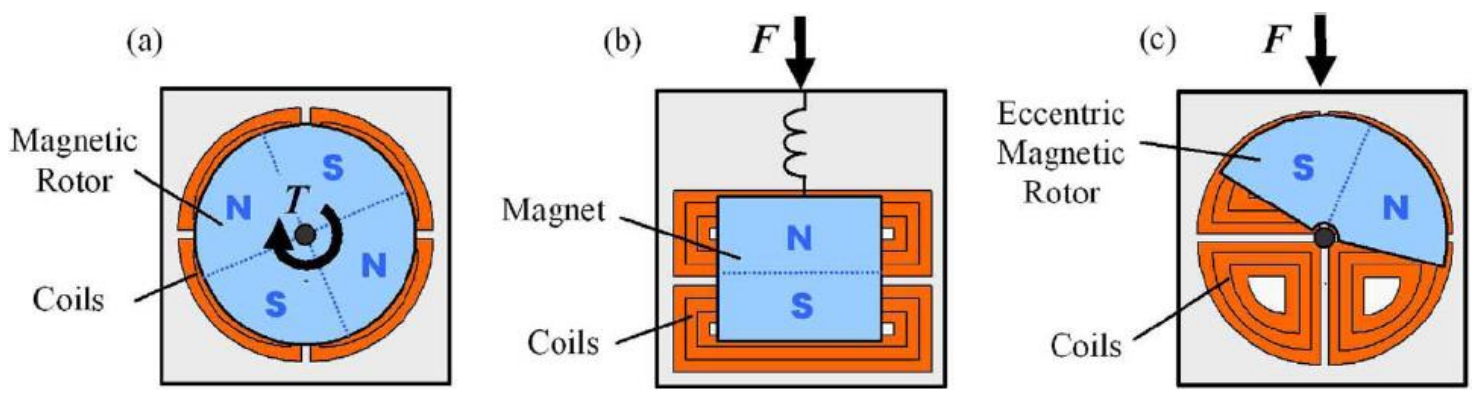

Figure 2-(a) rotational generator (b) oscillatory generator (c) hybrid generator [6]

\subsubsection{Existing Technologies/Prototypes}

The first MEMS electromagnetic design, in Figure 3, was published by Sherwood and Yates in 2003. This design consisted of a magnet attached to a $2.5 \mathrm{~mm}$ diameter diaphragm. A coil made of gold was placed below the magnet. Vibrations in the device base moves the magnet causing 
changes in the magnetic field which in turn induces an electric current in the coils. The maximum power generated was $0.3 \mu \mathrm{W}$ with a load resistance of $39 \Omega \mathrm{s}$; the reasons cited for such low output was due to nonlinear spring characteristics [7].

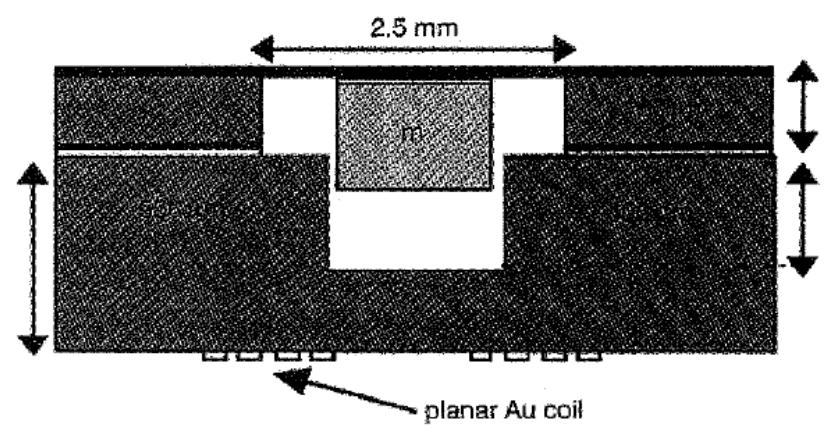

Figure 3 - Diaphragm electromagnetic generator [7]

In 2007 another electromagnetic device was designed with a fixed permanent magnet and an array of cantilever beams of 3 beams by 3 beams. They attempted to increase the power generated by using more beams as seen in Figure 4. A coil was embedded into each beam and a permanent magnet was placed beside the beams to generate a magnetic field. The researcher predicted only $6 \mathrm{nW}$ to be generated from each beam. They concluded that this type of electromagnetic generator did not perform well [8].

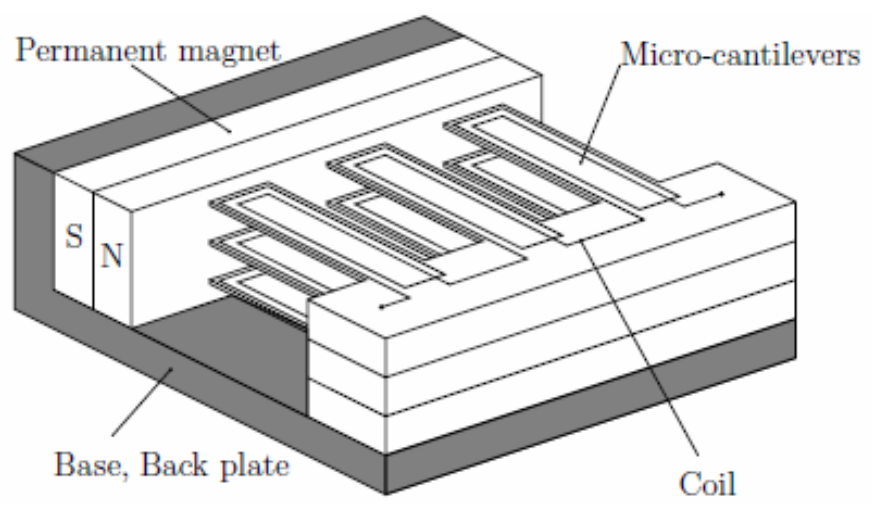

Figure 4 - Electromagnetic beam array [8]

Prior to 2004, electromagnetic designs had resonant frequencies in the kilohertz range, which was greater than most ambient vibrations which occurred at around 1-10Hz. During this year, researchers developed a rather ingenious design which consisted of two diaphragms placed on top of each other, this can be seen in Figure 5. The top diaphragm held a permanent magnet which 
could resonate at ambient frequencies since its own frequency ranged between 1-100 Hz. Placed below the magnets were an array of cantilever beams each with a metal tip, when the magnet vibrated, it attracted the tips of the cantilever beams, pulling them upwards and then releasing them when the force of gravity overcame the magnetic force. Upon release, the cantilevers vibrated at their own resonant frequency ranging from 1 to $10 \mathrm{kHz}$. The researchers' design was tested at the millimetre scale; however their proposed design had dimensions of $400 \mu \mathrm{m}$ by $200 \mu \mathrm{m}$ by $10 \mu \mathrm{m}$. The expected performance of a single beam was to generate $2.5 \mu \mathrm{W}$.[9]

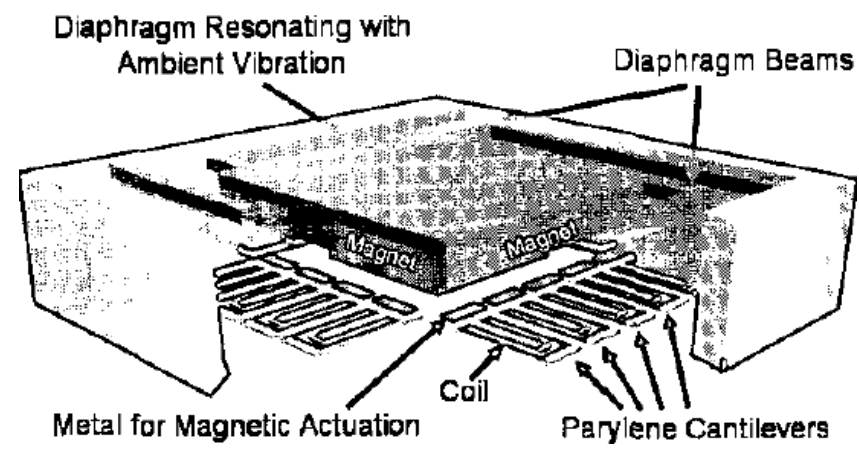

Figure 5 - Low frequency electromagnetic generator [10]

In 2007, another design aiming for a low resonant frequency was reported to achieve $52 \mathrm{~Hz}$. In the design, which is illustrated in Figure 6, a cantilever beam was fixed to a base made of rigid plastic and the coil with 600 turns of copper wire was placed onto the base. The maximum power generated was $46 \mu \mathrm{W}$ with a load resistance of $4 \Omega \mathrm{k}$. The power density of the device was calculated to be $307 \mu \mathrm{W} / \mathrm{m}^{3}$. [10]

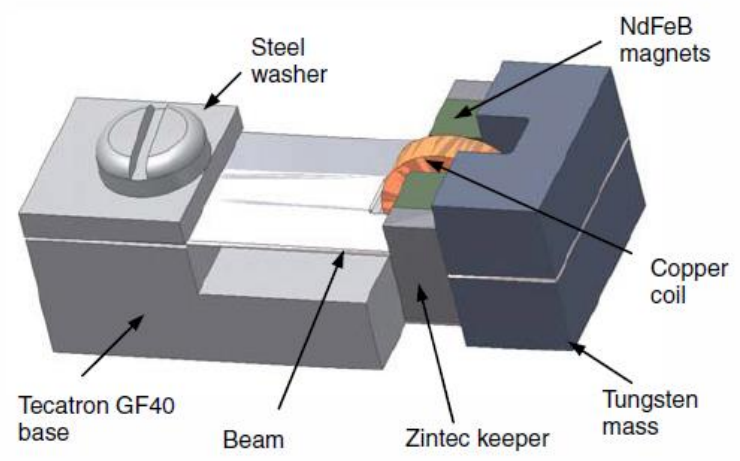

Figure 6- Micro Generator [10] 
One major issue with electromagnetic generators was the low number of turns per coil. This was solved using low temperature ceramic compounds where coils were placed in multiple layers of ceramic compound. This greatly increased the coil density per unit volume. [11]

In 2008, another cantilever design was produced to address the significant power drops associated with excitation at non-resonant frequencies. The solution to this hurdle was to create a device based on the cantilever beam design. The device contained thirty five parylene cantilever beams of varying lengths with a single copper coil layer. By varying the length of the beams the frequency range over which the device could operate was increased. An illustration of the device is included in Figure 7 and a photograph of the actual device is Figure 8. The device's range of operation was 4.2-5.0 kHz. It had a maximum power of $0.4 \mu \mathrm{W}$. However, the authors did mention that the maximum power was reduced due to the variable lengths of the beams.[12]

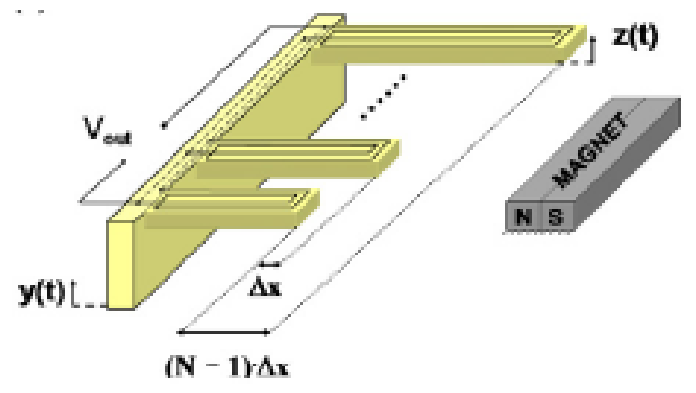

Figure 7- Wide bandwidth device design [12]

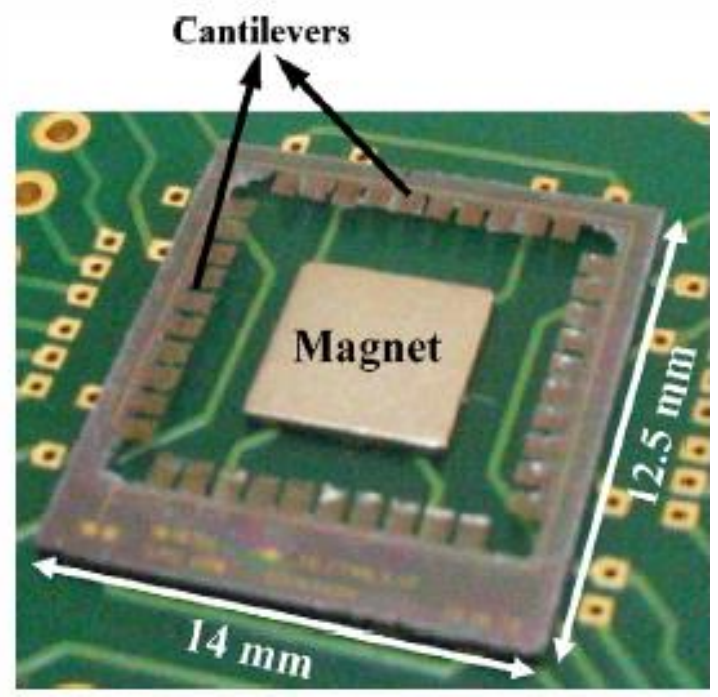

Figure 8-Fabricated wide bandwidth device [12] 
Another method for widening the harvester's bandwidth of frequencies was the use of stoppers. Stoppers increased the damping and stiffness of cantilever beams upon impact thereby transforming the system from a linear to piece-wise linear system. The findings of a paper in 2008 demonstrated that using a stopper which reduced the harvester's average power but increased the bandwidth by $240 \%$. The bandwidth widening was only observed when the researchers increased the frequency, but remained unaffected at lower frequencies. The paper also concluded that this method could be applied to electrostatic and piezoelectric harvesters as well [13]. Figure 9 shows an electromagnetic harvester with this new stopper method.

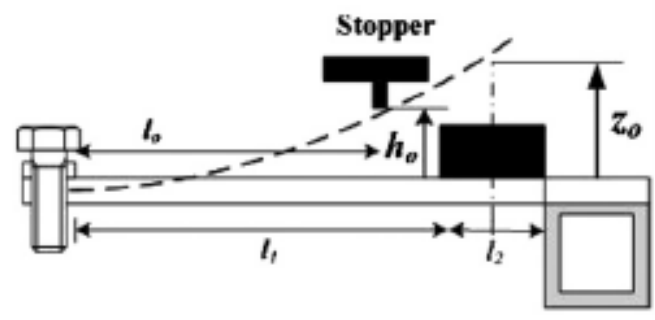

Figure 9- Electromagnetic harvester with stopper [13]

\subsection{Electrostatic Power Harvesters}

\subsubsection{Overview}

An electrostatic transducer works on the principle of capacitance. Essentially an electrostatic harvester is a variable capacitor. When two conductors with opposite charges are separated by a dielectric (insulator) an electric field exists between the two conductors and there is an electrostatic force attracting them. When there is vibration the plates are pulled apart and the distance between the two conductors increase. This adds energy to the system since work is done against the electrostatic force when the two conductors are moved apart. Figure 10 is an illustration of a capacitor and its plates with opposite charges. 


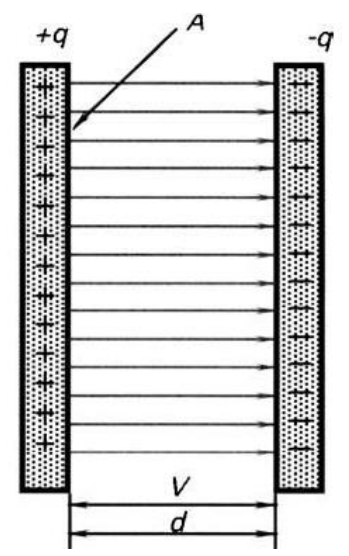

Figure 10-Capacitor [14]

There are two ways that the electrostatic harvester can be operated. One method is called charge constrained operation and the second method is voltage constrained. However both methods share three common operation steps. The first step is to place an electrical charge $Q_{0}$ on the variable capacitor when its capacitance is high $\left(C_{\max }\right)$. The capacitance is then reduced to a minimum $\left(C_{\mathrm{min}}\right)$ by moving the plates apart due to vibrations. Finally, the capacitor is discharged [15].

If the harvesting process were plotted on a charge versus voltage plot it would appear as shown below in Figure 11. The path A-B-D-A depicts a charge constrained conversion while the path AC-D-A depicts voltage constrained conversion. The net power harvested for each case is the area enclosed by their respective paths. [16] 


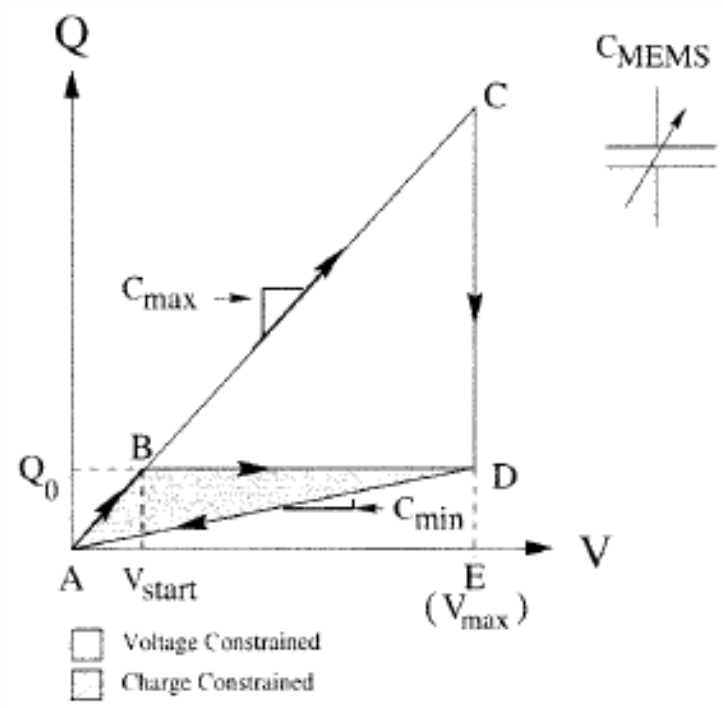

Figure 11 - Charge voltage plot with both charge and voltage constrained charging included [16]

\section{Voltage Constrained Case}

For the voltage constrained case, the first step is as described above; a charge is placed on the capacitor when the capacitance is at a maximum. The maximum capacitance occurs when the plates are closest together. The next step is to attach a voltage source/ battery to the capacitor to hold the voltage constant. The plates are then allowed to move apart to decrease the capacitance. While the plates are moving apart the charge will flow into the battery since the battery has lower impedance. This charges the battery [17].

The circuitry for the entire system is shown below in Figure 12, in Torres' paper the source was a rechargeable battery. The switches to activate different parts of the circuit for the three stages are controlled by a microprocessor and a logic circuit [18]. The logic circuit is shown in Figure 13. 


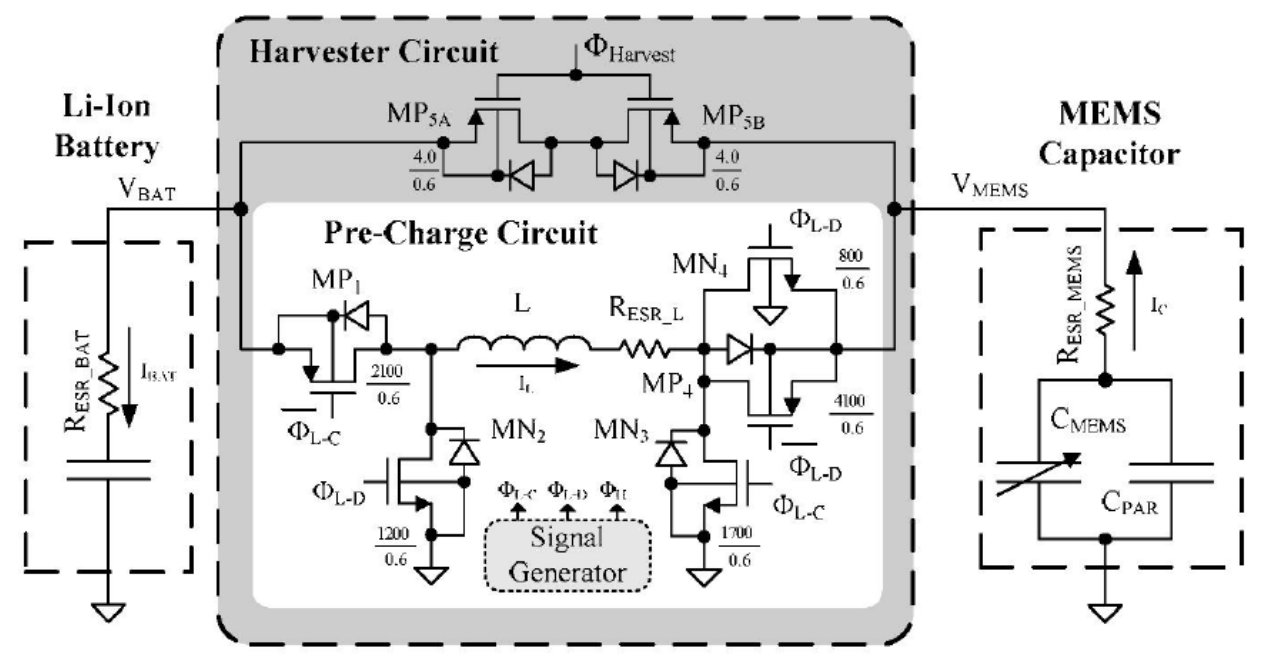

Figure 12- Circuit for a voltage constrained harvesters [18]

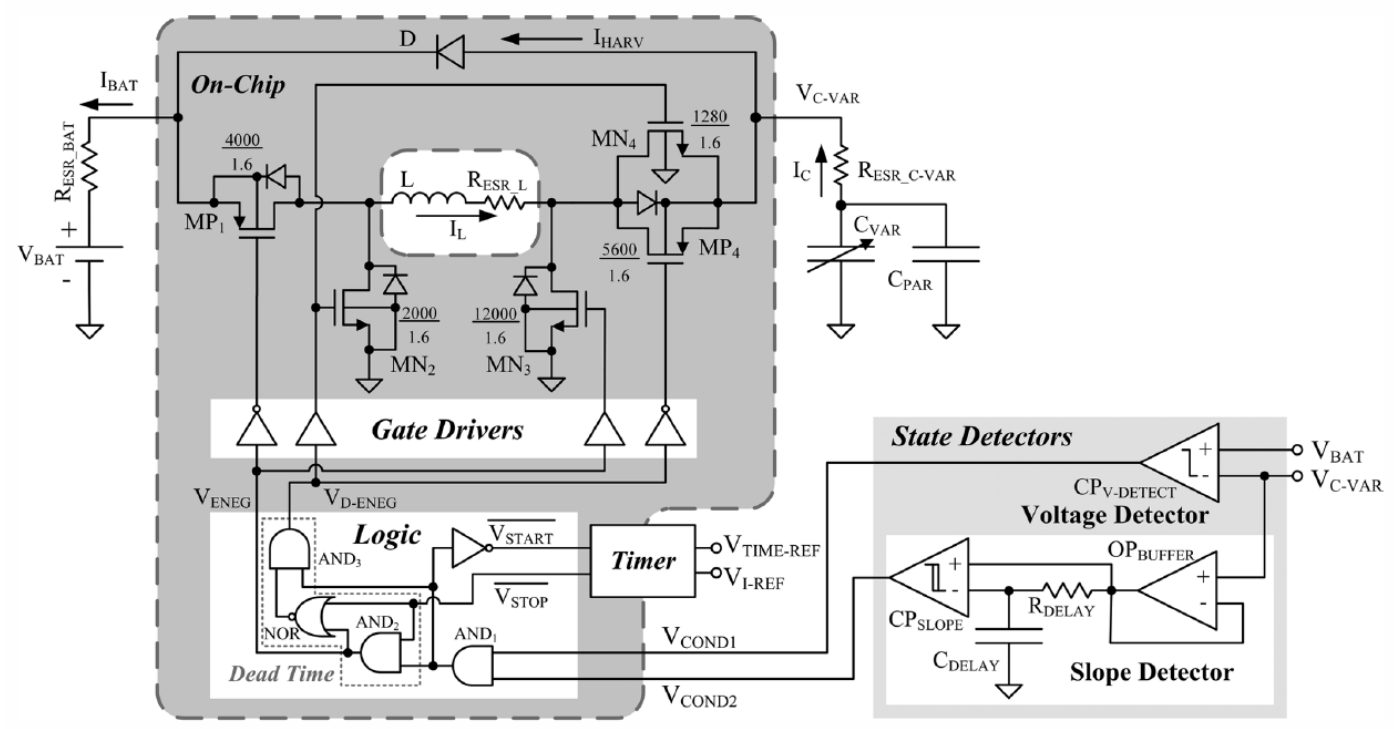

Figure 13 - Logic circuit to activate the switches [18]

Another method to hold the voltage constant is to use an electret. This is a material with a permanent electrification. To manufacture electrets, certain waxes, plastics or ceramic must be heated and then introduced to a strong electrical field to cool [19].

\section{Charge Constrained Case}

In the charge constrained case the harvesting cycle starts when the plates are close to each other and the capacitance is at a maximum $\left(\mathrm{C}_{\max }\right)$. At this point, a voltage source with voltage $\left(\mathrm{V}_{\text {in }}\right)$ is applied and the variable capacitor is charged. In the harvesting phase the voltage source is disconnected and the plates of the variable capacitor are allowed to separate from the excitation 
of the ambient vibration. The plates of the variable capacitor move apart and reach minimum capacitance $\left(\mathrm{C}_{\min }\right)$ at maximum separation; the potential difference across the plates is also maximized $\left(\mathrm{V}_{\mathrm{Max}}\right)[20]$.

The equations below describe the net energy harvested by the charge constrained method. $\mathrm{E}$ is the net energy and $C_{\text {par }}$ is the parasitic capacitance, which is the capacitance in the switches, wires and other parts of the circuit [20].

$$
\begin{gathered}
E=\frac{1}{2} V_{\text {in }}^{2}\left(C_{\max }-C_{\min }\right)\left(\frac{C_{\max }+C_{\text {par }}}{C_{\text {min }}+C_{\text {par }}}\right) \\
E=\frac{1}{2} V_{\text {max }} V_{\text {in }}\left(C_{\text {max }}-C_{\text {min }}\right)
\end{gathered}
$$

Figure 14 shows a circuit diagram of the charge constrained method. During the charging phase, switch one is closed to charge the variable capacitor. Once charged switched one is opened, disconnecting the voltage source. After the plates have separated due to vibration, the second switch is closed and the charge is transferred to the inductor and returned to the voltage source. The advantage of the method of charge constrain over the method of voltage constrain is that only one voltage source is required and the voltage does not have to be $\mathrm{V}_{\operatorname{Max}}$ [17].

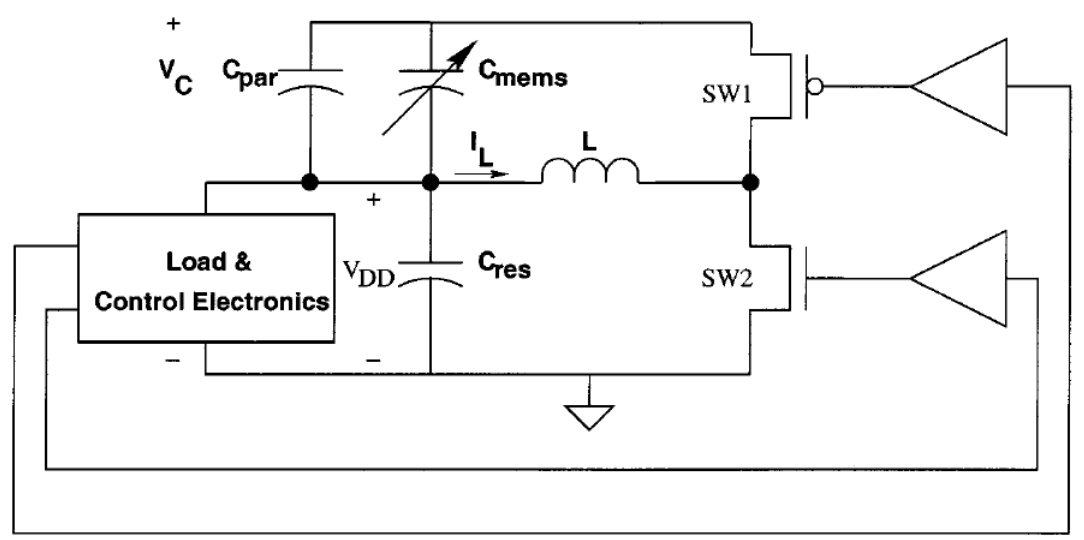

Figure 14 - Charge constrained circuit [17] 


\subsubsection{Existing Technologies/Prototypes}

In literature there are three main configurations or types of electrostatic harvesters which can be categorized as: in- plane gap closing type, in- plane overlap type, and out of plane gap closing type. The three configurations can be seen in Figure 15, Figure 16 and Figure 17 .

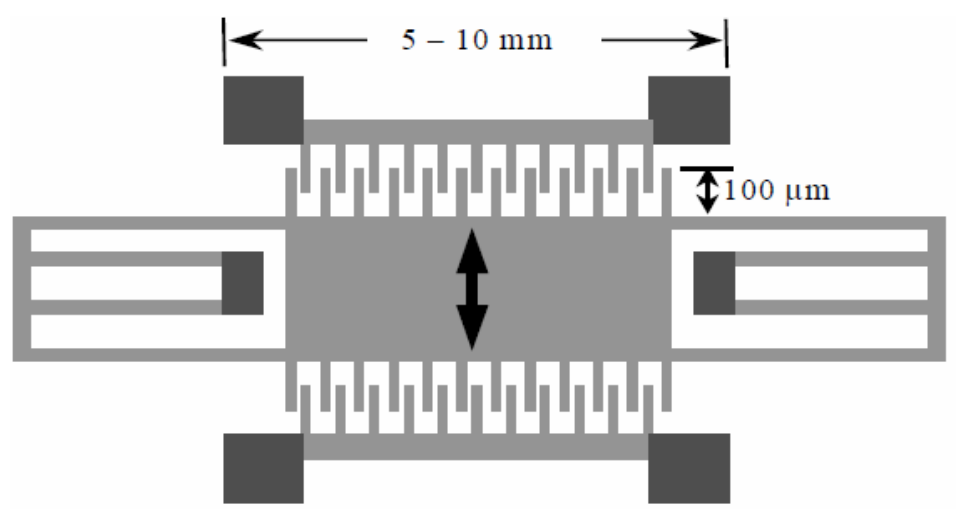

Figure 15- In-Plane overlap type [20]

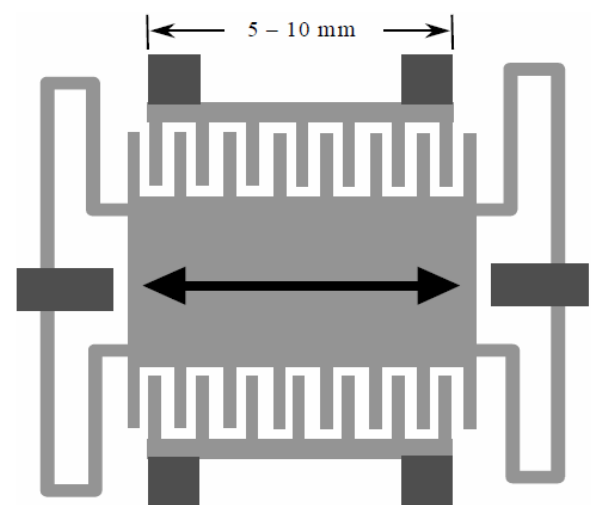

Figure 16- In-plane gap closing type [20]

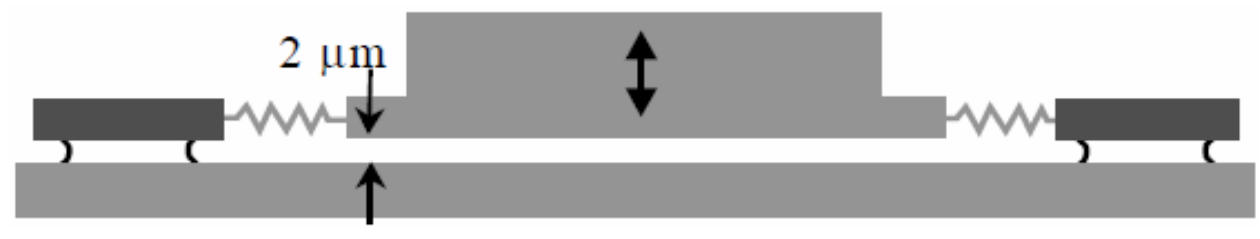

Figure 17- Out of plane gap closing type [20]

Each configuration has its advantages and disadvantages. For the out of plane gap closing type if plates get very close there is a possibility of the plates sticking together and causing short circuiting[20]. For the in plane gap closing configuration the advantage is that mechanical stops 
can be included to prevent short circuiting and control the maximum capacitance of the device [20]. For the in plane overlap type, stability is an issue. If the deflection is large and the gap is small, a small moment can cause fingers to touch and cause short circuiting [20]. For the gap closing types they operate using the constant charge method and the overlap type operates using constant voltage [21].

The first electrostatic device was described by Meninger et al in 1999. The paper explored the possibility of using a variable capacitor to generate electricity [16]. An illustration of Meninger's proposed design is shown in Figure 18.

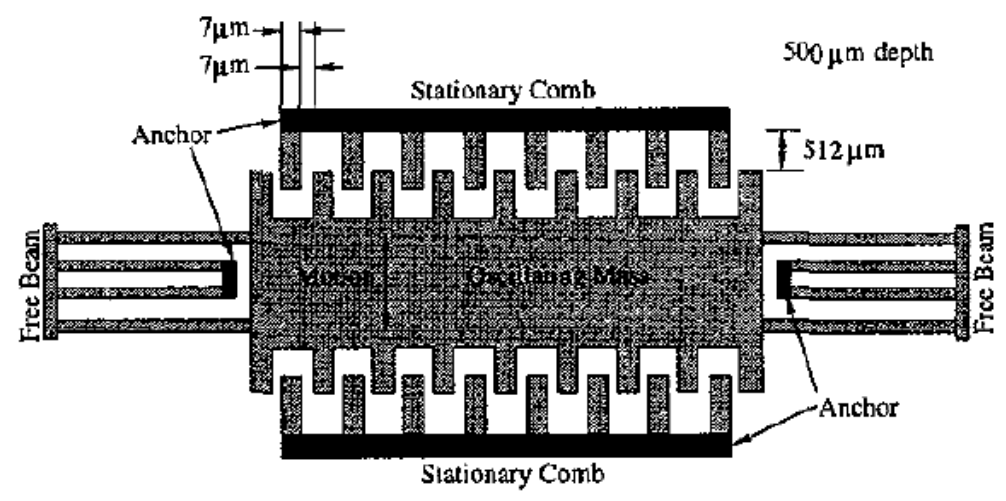

Figure 18 - First electrostatic harvester [17]

In a follow up paper in 2001 a prototype was built with dimensions of $1.5 \mathrm{~cm}$ by $0.5 \mathrm{~cm}$ and a resonant frequency of $2520 \mathrm{~Hz}$. The prototype produced $8 \mu \mathrm{W}$ of power [17].

Based on the equations the researcher concluded that in order to increase the power generated, the change in capacitance must be increased. Initially, the device was designed to reduce the gap between comb elements, but the gap size was constrained by the limits of fabrication technology. Other additional improvements include, increasing the height of device or lengthening the fingers of combs whilst taking into consideration the spring travel distance. It should also be noted that the more fingers added would increase the overall size of the device [17].

Another device was created based on the in-plane gap closing configuration. Table 1 shows the estimated results from models for their macro scale and micro scale devices. 
Table 1 - Results of Depesse's simulation [22]

\begin{tabular}{c|c|c|}
\hline Characteristics & $\begin{array}{c}\text { Bulk tungsten } \\
\text { macrostructure }\end{array}$ & $\begin{array}{c}\text { Silicon } \\
\text { microstructure }\end{array}$ \\
\hline \hline Size & $18 \mathrm{~cm}^{2} \mathrm{xl} \mathrm{cm}$ & $81 \mathrm{~mm}^{2} \times 0.4 \mathrm{~mm}$ \\
\hline Moving mass & $104 \mathrm{~g}$ & $2 \mathrm{~g}$ \\
\hline $\mathrm{C}_{\min } / \mathrm{C}_{\max }$ & $900 / 3590 \mathrm{pF}$ & $14 / 147 \mathrm{pF}$ \\
\hline $\begin{array}{c}\text { Resonant } \\
\text { frequency }\end{array}$ & $50 \mathrm{~Hz}$ & $50 \mathrm{~Hz}$ \\
\hline $\begin{array}{c}\text { Maximum } \\
\text { displacement }\end{array}$ & $116 \mu \mathrm{m}$ & $95 \mu \mathrm{m}$ \\
\hline $\begin{array}{c}\text { Maximum } \\
\text { scavenged power }\end{array}$ & $6 \mathrm{~mW}$ & $70 \mu \mathrm{W}$ \\
\hline
\end{tabular}

The actual results from the bulk tungsten macro scale prototype were only $1 \mathrm{~mW}$ of scavenged power. This is far lower than the estimated maximum scavenged power of $6 \mathrm{~mW}$. This result gives a conversion efficiency of $60 \%$. The conditions the device was tested under were with a sinusoidal vibration and amplitude of $90 \mu \mathrm{m}$ at a $50 \mathrm{~Hz}$ frequency. A micro scale silicon device has not been tested yet [22]. A photograph of the macro scale device is included as Figure 19.

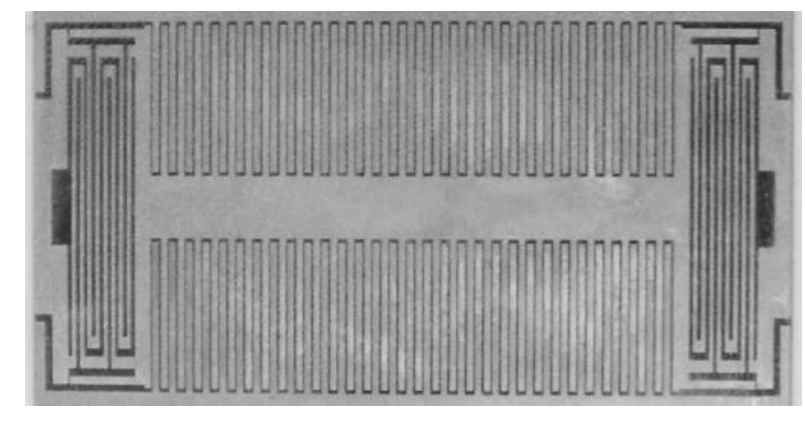

Figure 19 - Macro scale electrostatic harvester [22]

A new design was presented by the same authors as above, aimed to harvest energy from low frequency vibration from 0 to $200 \mathrm{~Hz}$ with low vibration levels of less than $1 \mathrm{~m} / \mathrm{s} 2$. The design, illustrated in Figure 20, contains many gaps and it allows the capacitance to vary continuously at the same amount. Although there were no power generated results from the prototype, the authors claim that this design can increase the conversion efficiency to 70 or $80 \%$. 


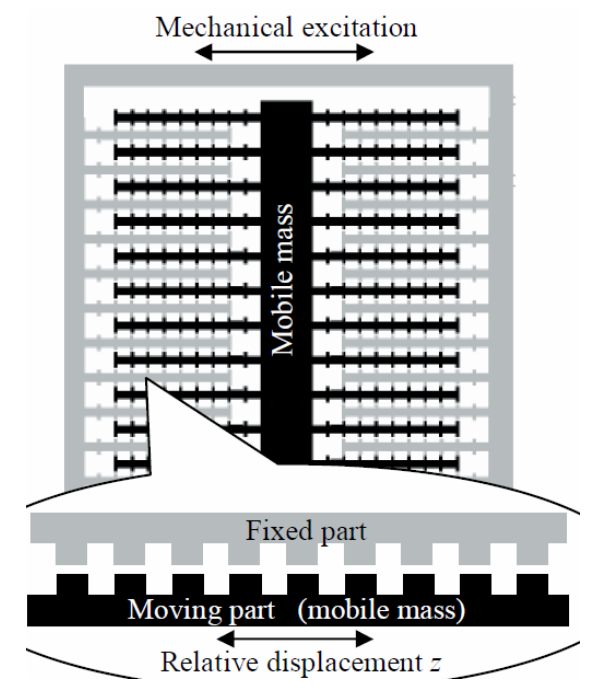

Figure 20 - Innovative electrostatic harvester [23]

Another group of researchers created a working device and an accurate model to predict the power output of the transducer. The total size of the device was only $5 \mathrm{~mm} \times 6 \mathrm{~mm}$. It produced $3.5 \mu \mathrm{W}$ of power, with two $560 \mathrm{k} \Omega$ resistors as the load. They are excited at their resonance frequency (1300-1500 Hz) with $13 \mathrm{~g}$ of acceleration [24]. A photograph of the entire actual fabricated device can be seen below in Figure 21. An illustration of electrostatic harvester is found as Figure 22.

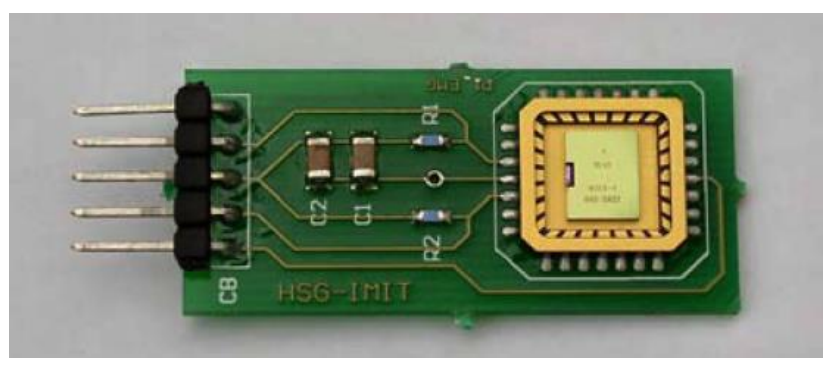

Figure 21 -Fabricated electrostatic harvester [24] 


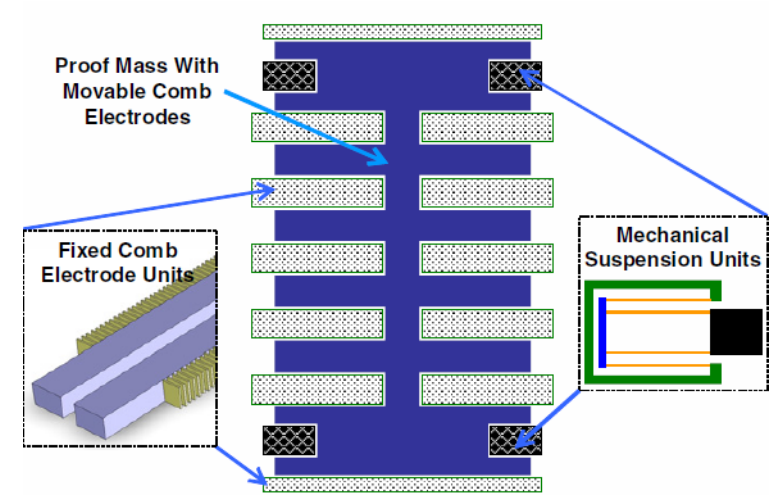

Figure 22 - Electrostatic harvester [24]

The next device found in literature claims to use a novel design for their device which did not use electrets to maintain the voltage difference during charging. This novel design is illustrated in Figure 23. Also an improved circuit was used to return the charge to the voltage source. The device produced $61 \mathrm{nW}$ with a $60 \mathrm{M} \Omega$ resistive load with a vibration level of $0.25 \mathrm{~g}$ at $250 \mathrm{~Hz}$. The overall size of the device was $66 \mathrm{~mm}^{2}$ [15].

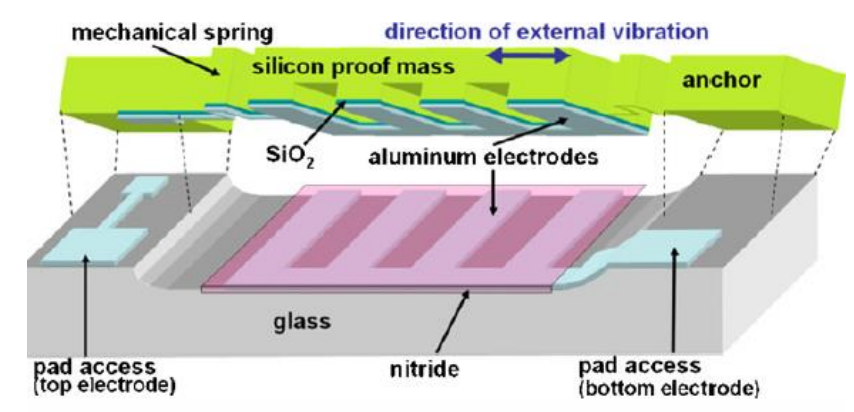

Figure 23 - Novel electrostatic harvester [15]

The most recent electrostatic harvester developed is an in plane device with rotary combs. The improvement this device had over previous devices was its low resonance frequency. Also because of its rotary design the device will capture the vibrations in the plane not just linear vibrations. The device had a resonance frequency of $110 \mathrm{~Hz}$, but this was reduced to $63 \mathrm{~Hz}$ when packaged in vacuum to reduce air resistance. The maximum electrical output at an acceleration level of $0.25 \mathrm{~g}$ was $0.39 \mu \mathrm{W}$ [25]. This rotary device can be seen below in Figure 24 . 

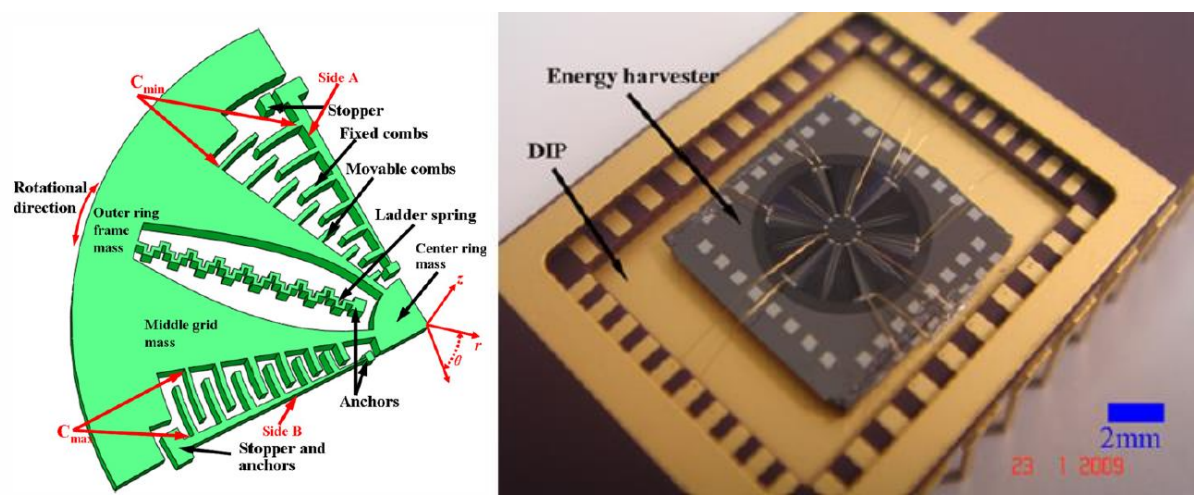

Figure 24 - Rotary electrostatic harvester [25]

\subsection{Piezoelectric Power Harvesters}

\subsubsection{Overview}

Piezoelectric transducers utilize the piezoelectric effect which is a phenomenon observed in certain materials which generate a voltage when stress or strain is applied[5]. The piezoelectric effect is a result of unsymmetrical distribution of negatively charged anions around cations at the molecular level. Deformation causes the anions to shift into an asymmetrical distribution around the cation, thereby forming opposing positive and negative ends. This is the basis of the piezoelectric effect. While there are naturally occurring piezoelectric materials such as quartz, they are not suitable for application in MEMS since the integration into a MEMS device is not feasible. In MEMS the piezoelectric materials used are part of a family of materials called ferroelectrics. Ferroelectrics have random polarization and must be poled prior to use as piezoelectric materials. This process involves heating the ferroelectric above its Curie temperature, immediately exposing it to a strong electric field and allowing it to cool. These materials can take on different forms including ceramics and polymer film [14]. Piezoelectric materials only respond to changing stress and the charge generated is proportional to the force applied. Also the properties of piezoelectric materials change logarithmically with age, stress and temperature and stabilize over time. The materials also have stress and temperature limitations (i.e. Curie temperature) and will lose their piezoelectric effect if these limits are reached.

Piezoelectric materials can be operated in either the 33 or 31 mode. The 33 mode means that both the stress and voltage are applied in the 3 direction; whereas the 31 mode has the stress 
applied in the 1 direction and voltage applied in the 3 direction. The direction in which the material is poled is known as direction 3 [2]. Figure 25 illustrates both 33 and 31 modes.
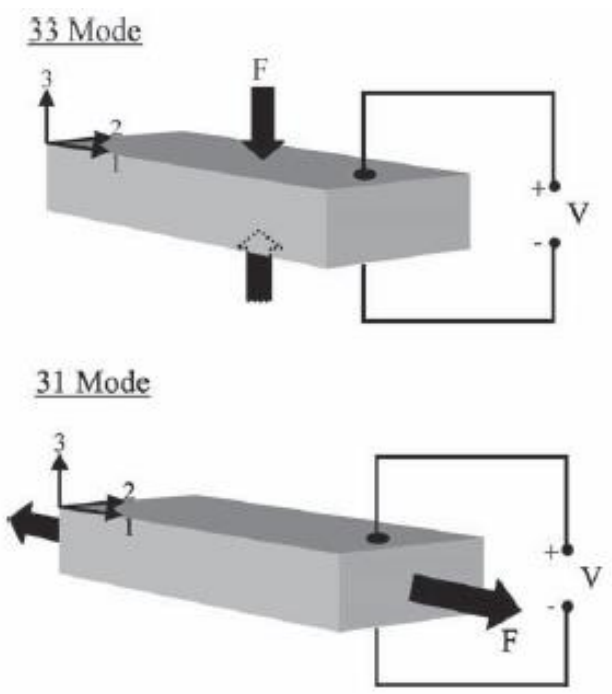

Figure 25 - Two modes of piezoelectric materials [2]

The piezoelectric effect takes two forms: the direct piezoelectric effect which gives the relation to find electric charge when mechanical strain is applied and the converse piezoelectric effect gives the relation to find the mechanical strain given the electric potential[26]. For a piezoelectric transducer only the direct piezoelectric effect is of concern. Below are the equations for both the direct and converse piezoelectric effect [5].

$$
\begin{aligned}
& \bar{D}=\epsilon^{\bar{\sigma}} \bar{E}+\mathbf{d} \bar{\sigma} \quad \text { direct piezoeffect, } \\
& \bar{\varepsilon}=\mathbf{S}^{\bar{E}} \bar{\sigma}+\mathrm{d}^{\mathrm{T}} \bar{E} \quad \text { inverse piezoeffect }
\end{aligned}
$$

The term $D$ is the vector of electrical displacement, $E$ is a matrix containing the coefficients of electrical permittivity, $\mathrm{E}$ is the electrical field vector, $\varepsilon$ is the strain vector and $\sigma$ the stress vector. The superscripts $\sigma$ and $\mathrm{E}$ indicate that the matrices are found at zero or constant stress $\sigma$ or electric field $\mathrm{E}$. The term $\mathrm{S}$ is the stiffness matrix and is made up of elastic coefficients. The term $\mathrm{d}$ is a matrix of direct piezoelectric coefficients or piezoelectric charge constants for which the formula is shown below. [5] 


$$
\begin{gathered}
d=\frac{\text { strain developed }}{\text { applied field }} \mathrm{m} / \mathrm{V}, \\
d=\frac{\text { short circuit charge density }}{\text { applied stress }} \mathrm{C} / \mathrm{N} \text {. }
\end{gathered}
$$

\subsubsection{Existing Technologies/Prototypes}

One of the earliest MEMS inspired piezoelectric power harvesters was created in 2003. Although more than $1 \mathrm{~cm}$ in length, it served as a proof of concept. It consisted of two layers of lead zirconium titante (PZT) serving as the piezoelectric material with a cantilever steel beam sandwiched in between. This configuration is known as a bimorph and was operated in the 33 mode [2].

As mentioned in the overview there are two modes of operation. The first 31 mode prototype appeared that same year and was $170 \mu \mathrm{m}$ in length with a resonance frequency of $13.7 \mathrm{kHz}$. The maximum power which could be harvested was $1 \mu \mathrm{W}$ with a $5.2 \mathrm{M} \Omega$ load. Unlike the bimorph design, the 31 mode design requires fewer layers [27]. The same authors published a paper in the following year with greater details of their design, including the obstacles faced during manufacturing. It was discovered that residual stress from the manufacturing process caused curvature in their fabricated beam. An inverse correlation was found between the elastic modulus of the base layer and the curvature of the beam. If the elastic modulus of the base layer is larger the resulting curvature is reduced [28].

The same year another group of researchers published a paper detailing a simulation of a macro scale bimorph configuration. For piezoelectric power generation, the electricity produced had to be rectified in order to create a direct current. The dimensions of their proposed device, illustrated in Figure 26, were $5 \mathrm{~mm}$ by $1 \mathrm{~mm}$ by $0.1 \mathrm{~mm}$. 


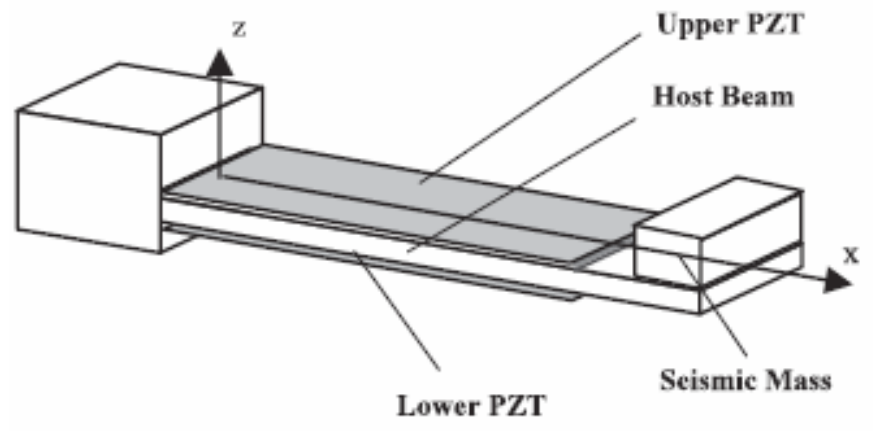

Figure 26 - Bimorph configuration [29]

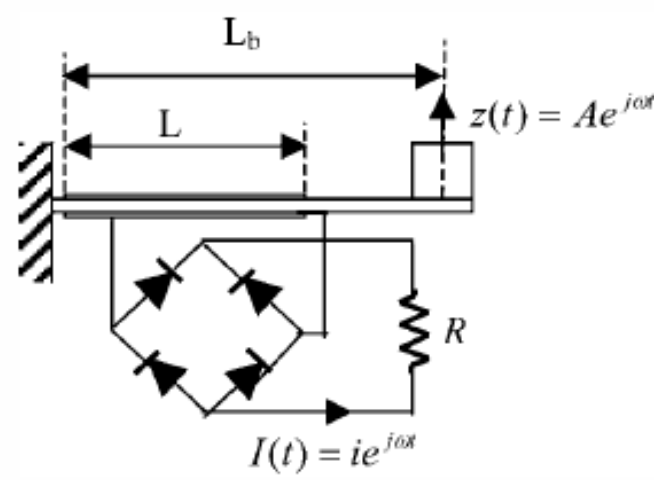

Figure 27 - Rectifier circuit [29]

From their simulation it was found that the power generated was proportional to the frequency of the vibration. The higher frequencies increase the maximum power value that can be reached. A graph depicting the power versus resistance curve at varying frequencies. As shown in Figure 28. Curves 1, 2, and 3 are at $7000 \mathrm{~Hz}, 4000 \mathrm{~Hz}$ and $2939 \mathrm{~Hz}$ respectively. [29]

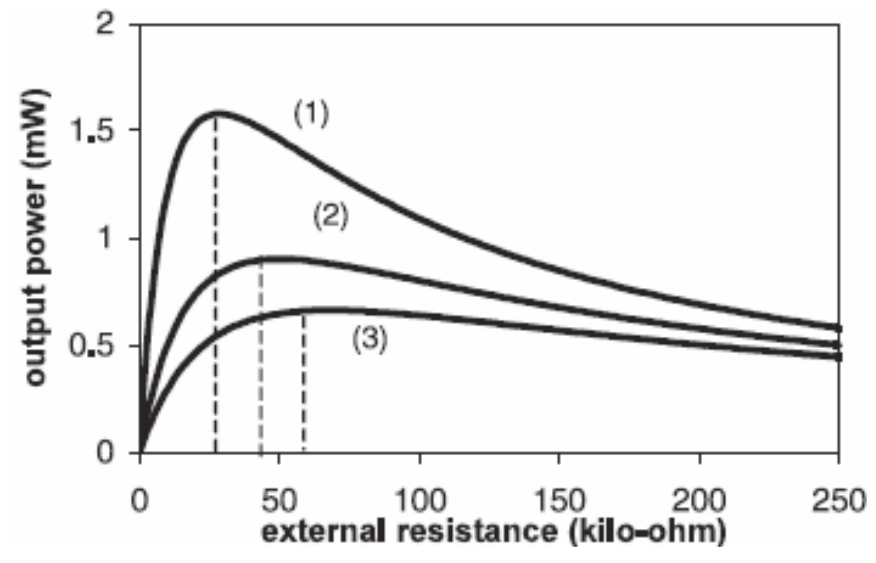

Figure 28 - Power vs. resistance [29] 
The maximum power generated was $1.6 \mathrm{~mW}$ when excited at $7000 \mathrm{~Hz}$. The curves' maxima indicate optimal external resistance values that correspond to maximum power output. The graph, Figure 29, plots power versus frequency with curve a representing a PZT PI515 material and curve b representing PZN-8\% PT. From the graph the researchers concluded that increasing frequency only improves the output power up to a certain point at which it starts to plateau despite higher frequencies. [29]

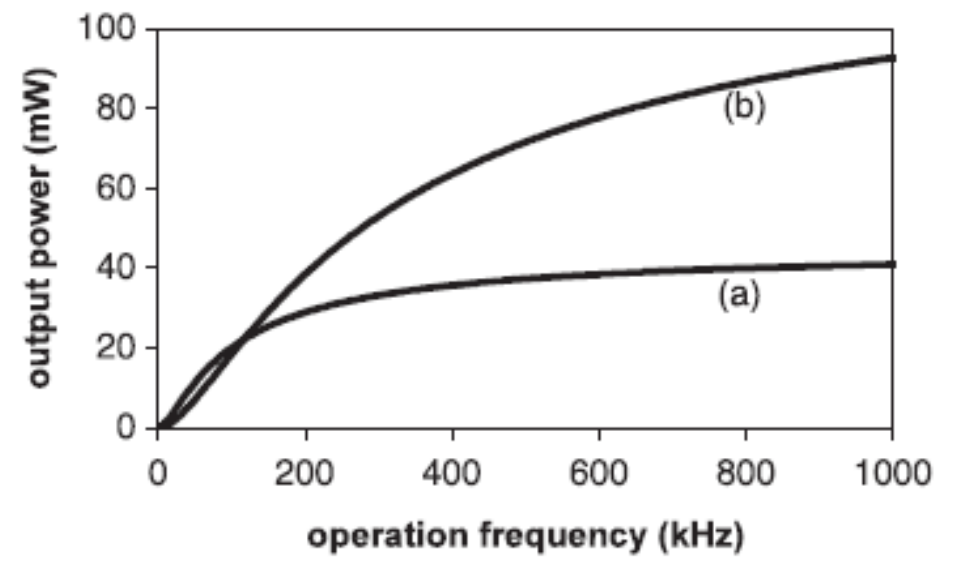

Figure 29 - Power vs. frequency [29]

Sood, Jeon, Jeong and Kim published a paper in 2005 [30] detailing their design, the inspiration of which came from advances in piezoelectric sensors where a method was found to utilize the 33 mode for electric power harvesting. Shown below Figure 30 are the electrode placements for the 31 mode vs. the 33 mode.
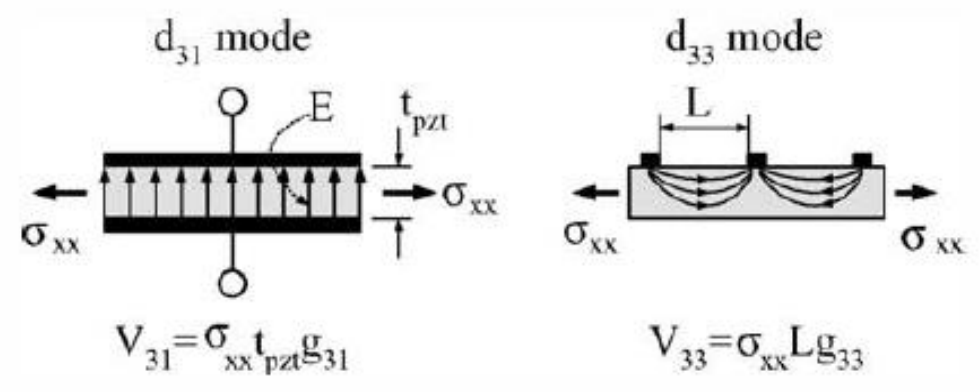

Figure 30 - 31 vs. 33 modes [31]

The 33 mode has a superior relationship between strain (x) and electric field (E) and stress $(\sigma)$ and voltage $(\mathrm{V})$ of piezoelectric materials. This is due to the difference in the piezoelectric 
constants and the structure of the device. The terms $\mathrm{d}$ and $\mathrm{g}$ are piezoelectric constants and $\mathrm{L}$ is distance between electrodes. The equations shown below are the basic piezoelectric equations introduced previously in the overview [30].

$$
\begin{aligned}
& x_{3}=d_{3 i} E_{i} \\
& V_{3 i}=\sigma_{x x} g_{3 i} L_{i}
\end{aligned}
$$

The term $\mathrm{L}$ which represents the separation distance of the electrodes can be larger for the 33 mode harvesters since the electrodes are applied as inter-digitated combs. But for the 31 mode the electrodes are sandwiched between the piezoelectric materials, thus $\mathrm{L}$ is the thickness of material. Also d33 and g33 are 2 to 2.5 times larger for most materials. Thus the open circuit voltage of the 33 mode could be almost 20 times greater. Shown below, in Figure 31, is the layout of a 33 mode harvester.

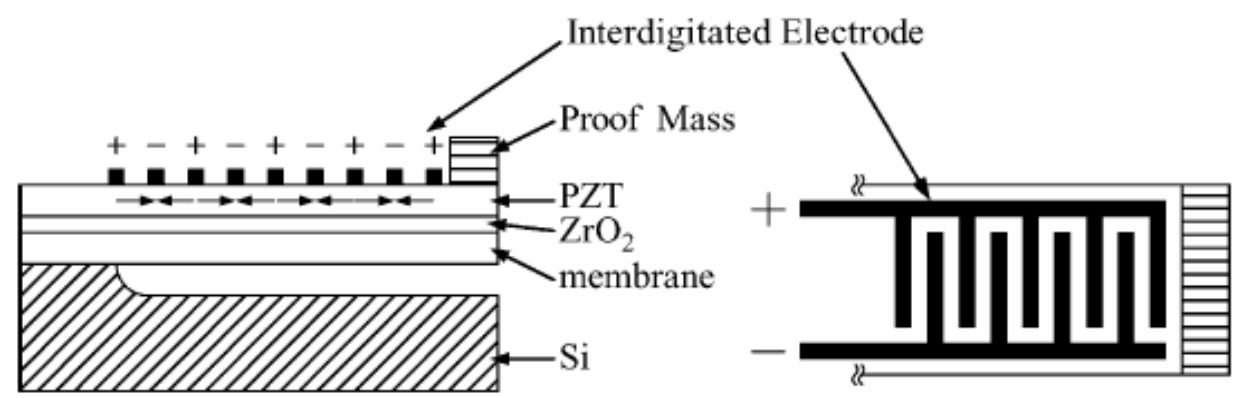

Figure 31 - 33 mode harvester [31]

A scanning electron microscope (SEM) photo of the MEMS scale 33 mode harvester with interdigitated combs as shown below in Figure 32. The results were the same as their 2003 paper and the energy density of their device is $0.74 \mathrm{mWh} / \mathrm{cm}^{2}$ including the circuit[31]. 


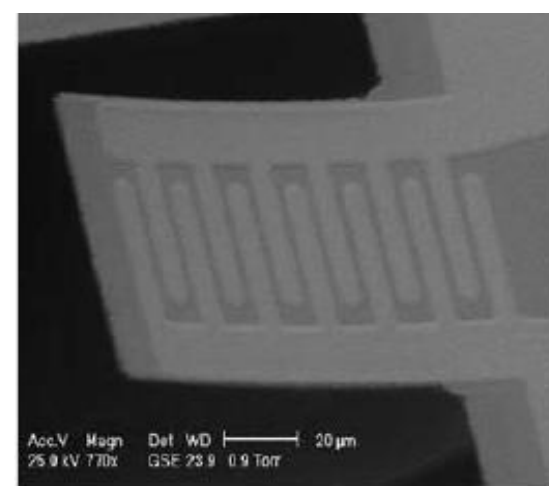

Figure 32 - SEM photo of interdigitated electrodes [31]

The power harvesting circuit for piezoelectric harvesters is also important, thus Ammar et al. in 2005[32], investigated if connecting devices in series or parallel made a difference. Although the experiments were conducted on a macro scale, the findings of the research remain relevant. It was discovered that $11.4 \mathrm{nW}$ of power was generated at an optimal resistance of $12.5 \mathrm{M} \Omega$ for harvesters connected in series and $11.6 \mathrm{nW}$ of power was generated at the optimal resistance of $333 \mathrm{~K} \Omega$ for harvesters connected in parallel. The results demonstrate that for parallel connection of harvesters the load resistance required to generate the maximum power is significantly lower than harvesters connected in series. [32]. Figure 33 shows Ammar's MEMS power harvester.

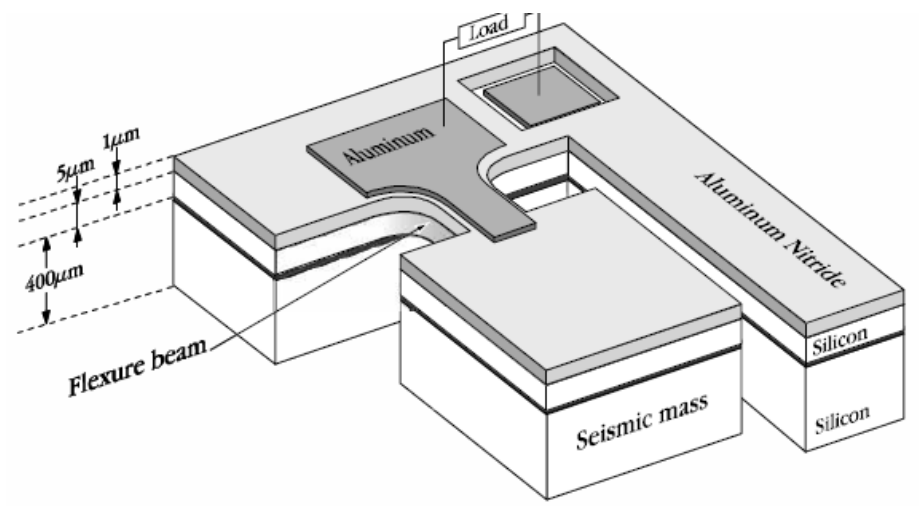

Figure 33 - Piezoelectric harvester [32]

Although the research focused on the harvesting circuit, a piezoelectric harvester was also fabricated with a cantilever design and a seismic mass made of silicon. The piezoelectric material chosen for the device was aluminum nitride. Figure 34, below is the circuit designed to rectify and to charge an attached battery. [32] 


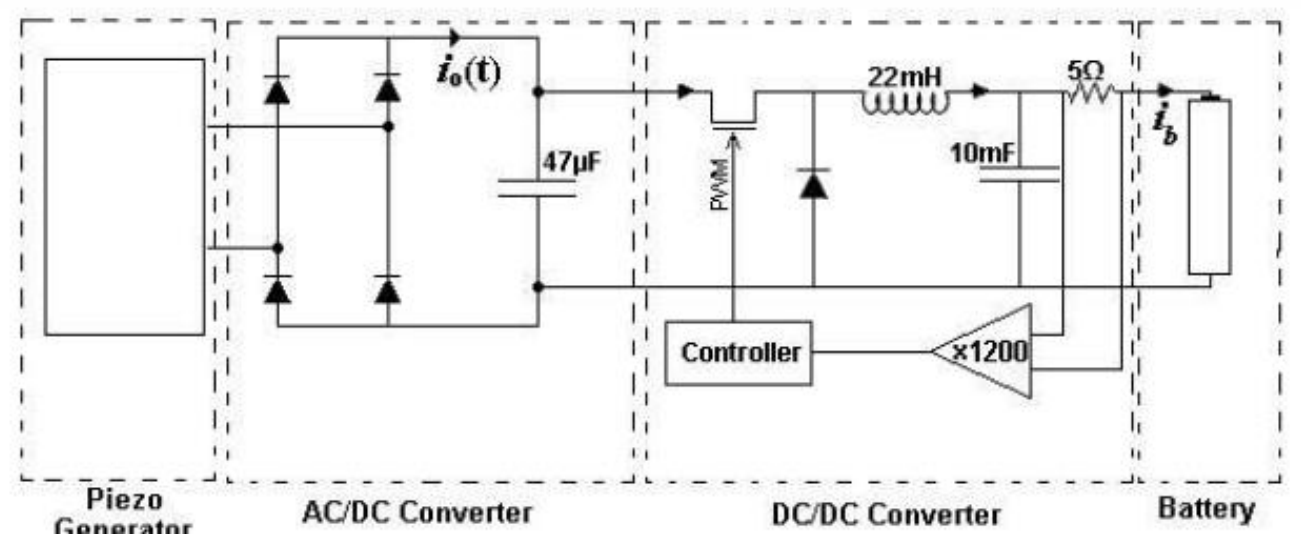

Figure 34 - Battery charging circuit [32]

The first attempts at creating a power harvester that automatically tuned itself to ambient vibrations was documented and published in a paper in 2005 by Shad Roundy and Yang Zhang. The researchers attempted to use a feedback loop in order to continuously tune the device. From their model it was shown mathematically that active tuning would result in a net loss in power. They fabricated a device based on a cantilever beam with both an actuator and transducer on the beam. Although the results from their prototype did not match the model, the results did show the same general pattern as the model [33]. Their self-tuning device is seen below in Figure 35.

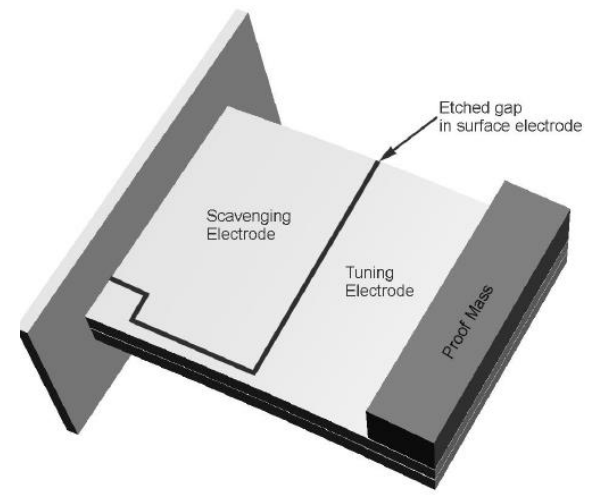

Figure 35 - Active tuning device [33]

The next device presented used nickel for the proof mass. A proof mass is a weight placed at the end of the beam, included to lower the natural frequency of MEMS power harvester. It is an early MEMS based device based on Roundy's design [34]. The piezoelectric material utilized is a PZT film of $1.5 \mu \mathrm{m}$ thick deposited with the Sol Gel method. The total length of the device is $2 \mathrm{~mm}$ long and produced a $1.15 \mu \mathrm{W}$ of power with a load of $20.4 \mathrm{k} \Omega$ at $1 \mathrm{~g}$ of acceleration. The 
resonance of this device is $609 \mathrm{~Hz}$ [35]. Figure 36 shows an illustration of the device and Figure 37 is a SEM photo of the actual device.

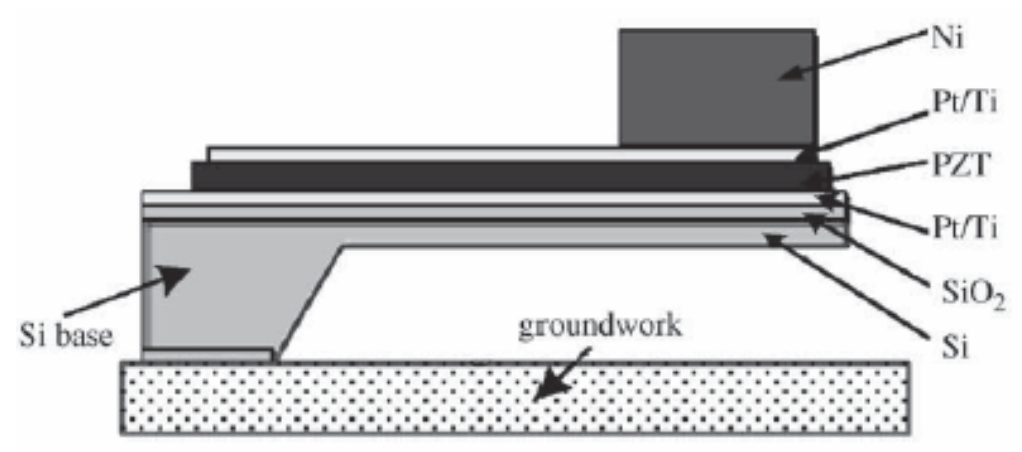

Figure 36 - Nickel proof mass [34]

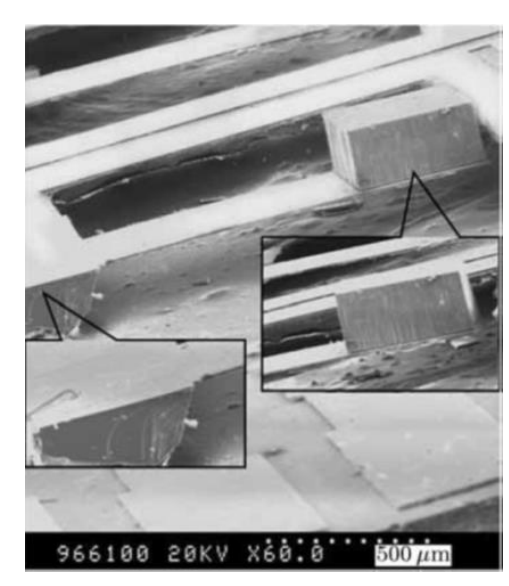

Figure 37 - SEM photo of device and nickel mass [35]

In 2008 another solution to the low frequency range problem was presented; the design consisted of a cantilever beam with a mass. In addition to the mass, magnets were placed on the free end of the beam and also above and below the free end of the beam to provide additional stiffness to the beam as illustrated in Figure 38. The magnets on the top provided an attractive force whilst the magnets on the bottom provided a repulsive force. As the magnets moved towards or away from the beam, the stiffness was altered thereby changing the resonance frequency. However the magnets had to maintain each position for a specific period of time prior to changing positions since continually doing so would result in a net loss of power. The device was able to achieve a $10 \mathrm{~Hz}$ bandwidth from $22-32 \mathrm{~Hz}$. The range of the power harvested was between $240-280 \mu \mathrm{W}$ [36]. 


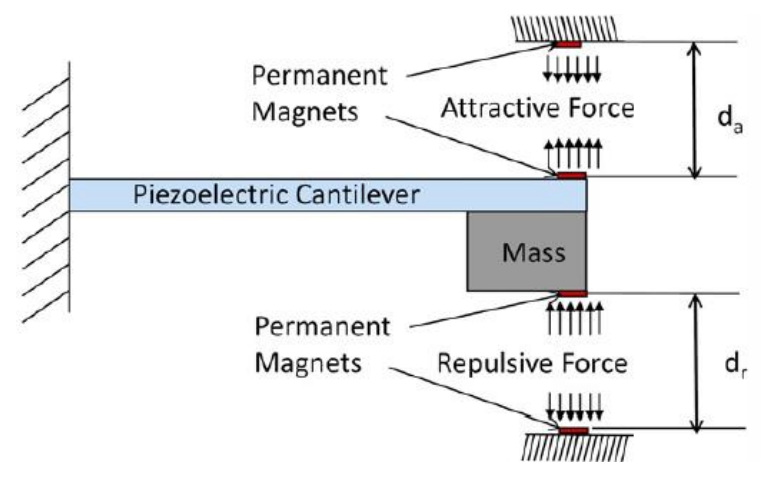

Figure 38 - Magnet tuning harvester [36]

The previous MEMS design relied on an additional manufacturing step to add a nickel mass to the beam. The new design, in Figure 39, simplified the manufacturing by utilizing the silicon wafer as the mass without having to add extra material. The silicon wafer was backside etched for use as a mass. The overall structure remains as a cantilever beam with a mass to achieve a resonant frequency of $461.15 \mathrm{~Hz}$. The dimensions approach MEMS scale with a length of 4.8 $\mathrm{mm}$ and a width of $0.4 \mathrm{~mm}$. The power produced was $2.15 \mu \mathrm{W}$ at $2 \mathrm{~g}$ of acceleration with $6 \mathrm{k} \Omega$ load [37].
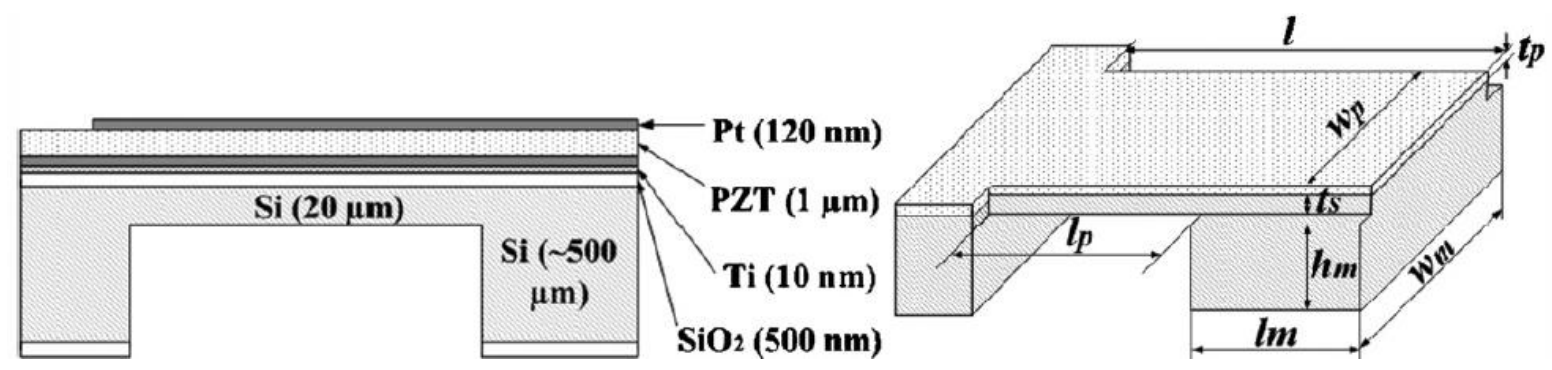

Figure 39 - Silicon mass cantilever beam [37]

Another 33 mode device, illustrated in Figure 40, was created with a cantilever design with dimensions $0.8 \mathrm{~mm}$ by $1.2 \mathrm{~mm}$. The piezoelectric material only covered an area with dimensions of $0.8 \mathrm{~mm}$ by $0.4 \mathrm{~mm}$. A voltage of $1.6 \mathrm{~V}$ and an output power of $1.4 \mu \mathrm{W}$ were reported with $2 \mathrm{~g}$ of acceleration and a frequency of $870 \mathrm{~Hz}$. Some findings of this paper are that the Sol Gel method creates the best piezoelectric layer. The voltage requirement to rectify the AC signal of the power harvester was $0.5 \mathrm{~V}$. [38] 


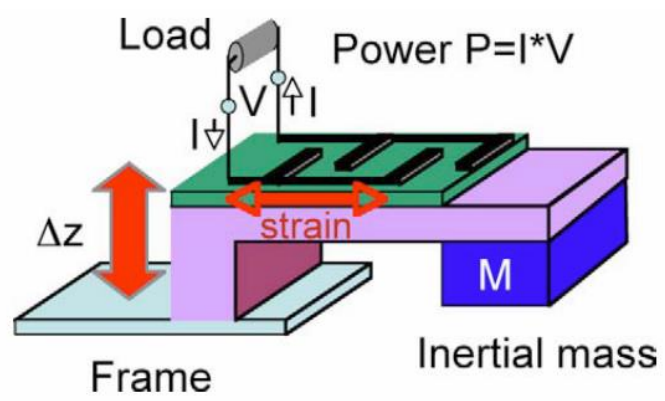

Figure 40 - Another 33 mode [38]

To determine which mode performed better in reality, two harvesters with the same dimensions but different modes were fabricated and tested. The harvesters used in the testing are shown below in Figure 41. It was found that the 31 mode had better performance despite equation 2.4 indicating otherwise. This was due to the 31 mode's superior poling and the fact that the areas under the electrodes were not poled. The 31 mode piezoelectric MEMS generator had a maximum open circuit peak to peak output voltage of $2.675 \mathrm{~V}$. When a resistor was connected the maximum output power was found to be $2.765 \mu \mathrm{W}$ with a $1.792 \mathrm{~V}$ peak to peak output voltage during a resonant frequency of $255.9 \mathrm{~Hz}$ with $2.5 \mathrm{~g}$ of acceleration. The 33 mode piezoelectric MEMS generator had a maximum peak to peak open circuit voltage of $4.127 \mathrm{~V}$ and a maximum output power of $1.288 \mu \mathrm{W}$ with a $2.292 \mathrm{~V}$ peak to peak output voltage during a resonant frequency of $214 \mathrm{~Hz}$ with $2 \mathrm{~g}$ of acceleration. The maximum power output was more than double for the 31 mode in comparison with the 33 mode.[39]

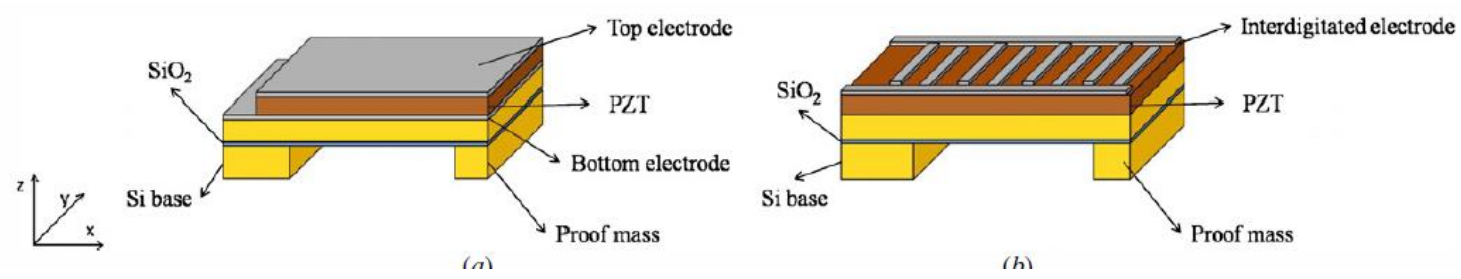

Figure 41 - 31 vs. 33 mode generators [39]

Although not an entirely piezoelectric system, the device designed by Tadesse, Zhang and Priya utilizes a clever combination of piezoelectric and electromagnetic properties. Their design can be seen in Figure 42. Based on the cantilever beam model, several piezoelectric crystals are placed along the length of the beam and the permanent magnet tip doubles as a tip mass for lowering the resonance frequency of the system. As the magnet moves on the tip of the beam the coil around 
it will act as an electromagnetic generator. The size of their prototype was $25 \mathrm{x} 30 \mathrm{x} 125 \mathrm{~mm}^{3}$. The power generated from the electromagnet and piezoelectric was $0.25 \mathrm{~W}$ and $0.25 \mathrm{~mW}$ respectively. [40]

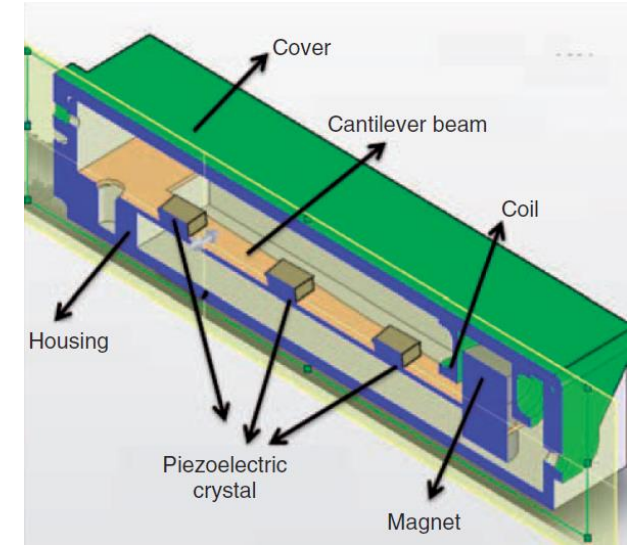

Figure 42 - Magnetic and piezoelectric hybrid harvester [40]

Another method for tuning the natural frequency of a beam to match the excitation frequency is by applying an axial load to the beam. One design utilizes a screw that applies a force to wings that are connected to arms, which are in turn connected to the tip of the beam as illustrated above. The aim of the paper was not focused on the amount of power harvested but rather the feasibility of tuning by applying an axial load. The team found the idea to be feasible and demonstrated it with an experimental prototype. The researchers found that applying a compressive and tensile preload shifted the frequency $22 \%$ and $4 \%$ respectively [41]. Their design for applying an axial load is seen in Figure 43.

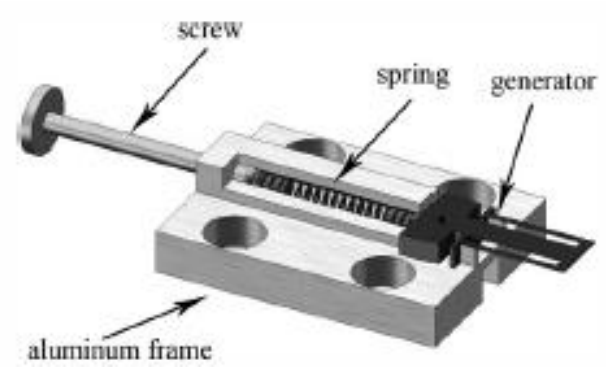

Figure 43 - Axial preload for tuning [41]

After investigating the optimal poling direction, Lee and his team decided to investigate whether serial or parallel poling was more advantageous for a bimorph. Two bimorphs of the same dimensions $\left(3000 \times 1500 \times 10 \mu \mathrm{m}^{3}\right)$ were created one for serial poling the other for parallel poling. For parallel poling both layers of the piezoelectric material are poled in the same 
direction. The two layers are poled in opposite directions for serial poling. From their results, the conclusion was that serial poling provides more power however the optimal resistance is higher if the piezoelectric layers are connected in serial [42]. Figure 44 illustrated the poling direction for both parallel and serial polarization methods.

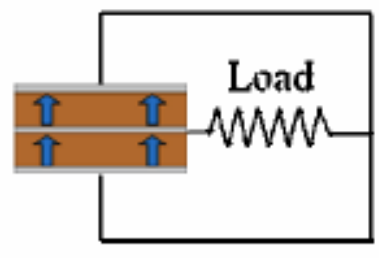

(a) parallel polarization

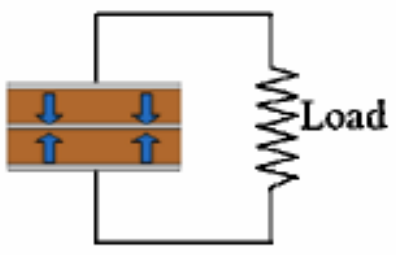

1: Poling direction

(b) serial polarization

Figure 44 - Poling directions [42]

Expanding on Soliman's work on stoppers in 2008, researchers simulated the effect of stoppers for piezoelectric devices. Their work numerically confirmed the fact that stoppers, modeled as springs and a damper, can increase harvester bandwidth. They also proposed a piezoelectric design, illustrated in Figure 45, and used AlN instead of PZT as the piezoelectric material, claiming that AIN did not require poling and was comparable to PZT. [43]

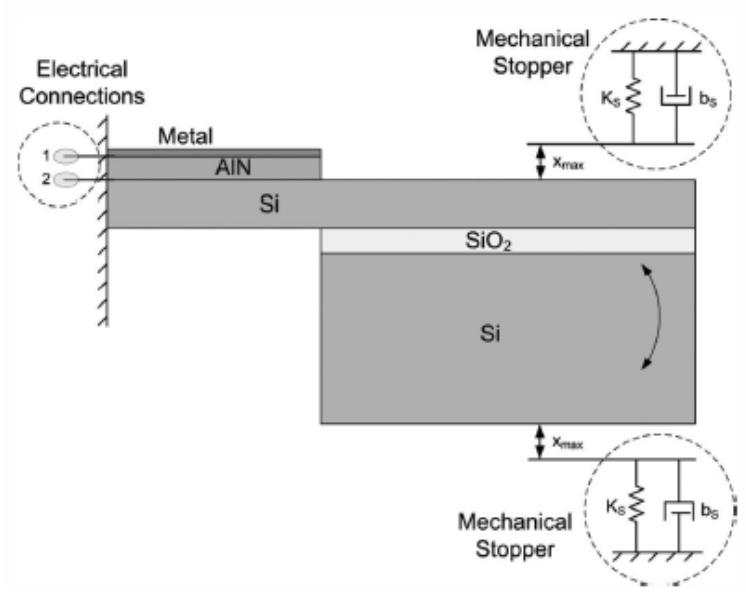

Figure 45 - AlN harvester with stoppers [43]

As with the electrostatic and electromagnetic actuators, the piezoelectric actuators faced the challenge of having a resonant frequency that matched the relatively low frequencies of ambient vibrations. The design presented below, in Figure 46, was able to achieve a very low frequency of only $35.8 \mathrm{~Hz}$. The large proof mass at the end was adjustable since it had many slots to tune the 
resonant frequency of the device to match the vibration source. Despite the lack of power output, a maximum open circuit voltage of $42 \mathrm{mV}$ was reported[44].

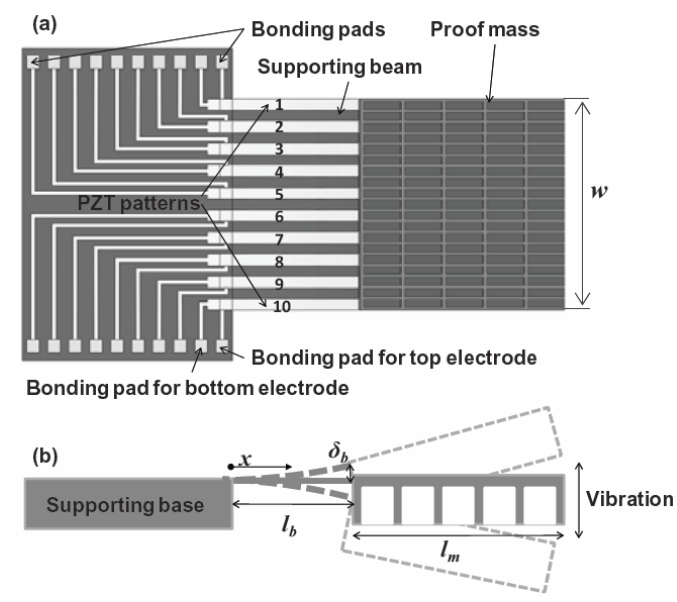

Figure 46 - PZT cantilever design [44]

Utilizing the stopper technique Liu presented another design. The ingeniousness of his design stemmed from the fact that the stoppers were composed of PZT beams, which would harvest electricity upon displacement. Although the stopper did not have a proof mass attached, during impact, the stoppers would be displaced and then left to oscillate at its natural frequency. The frequency range for this setup, illustrated in Figure 47, was $30-48 \mathrm{~Hz}$ with average peak to peak voltages of $0.23 \mathrm{~V}$ for PEH-B, the bottom beam with the proof mass. For PEH-T, the stopper beams, the average peak to peak voltage was $0.08 \mathrm{~V}$ [45]. In addition, Liu's group had published several papers in other journals discussing the same design.

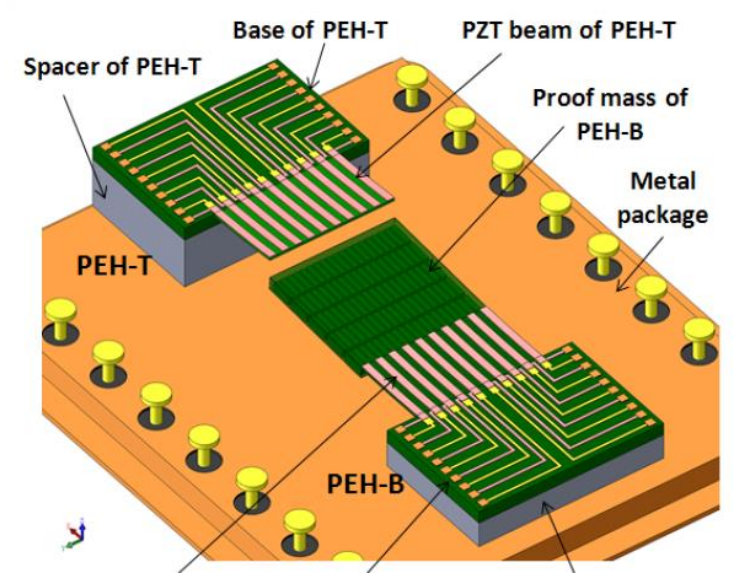

Figure 47 - Wideband harvester with stopper [45] 
In the search for broadening the range of harvesting frequencies, some researchers at MIT have designed a harvester that utilizes nonlinear springs, also known as a Duffing oscillator. The design used doubly clamped beams instead of cantilever beams. This resulted in having both stretching and bending in the beams. The stiffness of the structure is dependent on the stretching which is dependent on the amplitude of the vibrations. Thus the resonant frequency will in essence follow the excitation frequency. This enables the device to harvest frequencies at a wider bandwidth [46]. The reported power density of the device was $2 \mathrm{~W} / \mathrm{cm}^{3}$. The maximum power generated was $22 \mu \mathrm{W}$ and the optimal load resistance was found to be $290 \mathrm{k} \Omega$. No dimensions were given for the device; the authors only stated that it was the size of an US quarter [46]. Below is Figure 48 which illustrates the device utilizing Duffing oscillators.
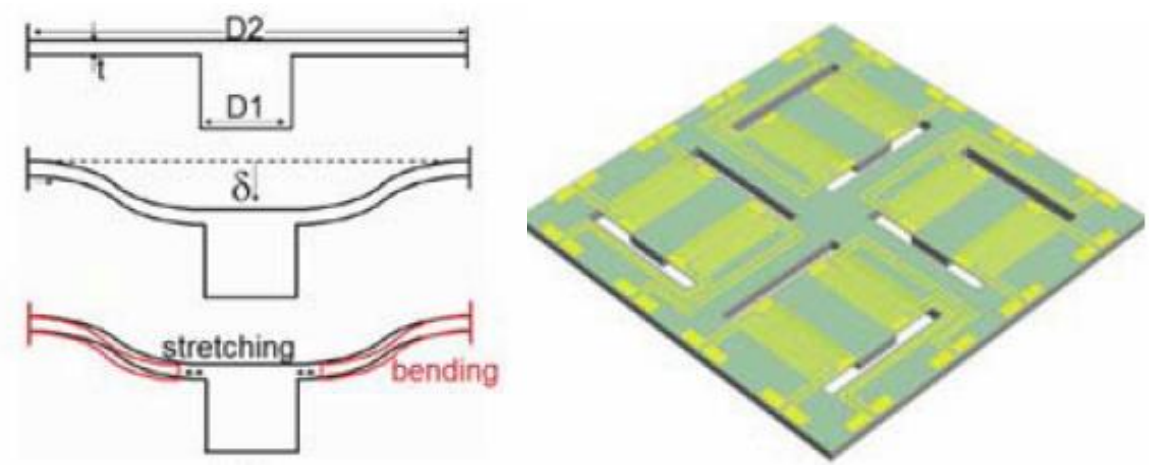

Figure 48 - Wide bandwidth harvester [46]

Furthering the work in 2009, the researchers focused this time on achieving a net positive gain on power harvested versus power used to tune and run the system. To find the proper preload for a certain frequency the team used a lookup table which was checked against the ambient vibration every 22 seconds. Also incorporated into their microcontroller was a learning process which was initiated every 4th cycle to improve the numbers in the lookup table. Thus their device, shown below in Figure 49, was able adapt and harvest from $150 \mathrm{~Hz}-190 \mathrm{~Hz}$ effectively and produce a net gain in power.[47] 


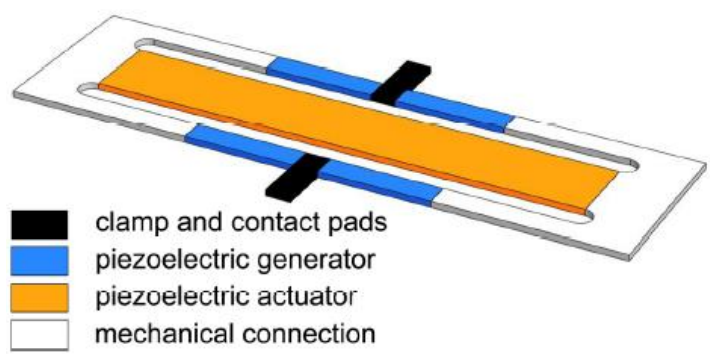

Figure 49 - Axial load tuning harvester [47]

In addition to nonlinear springs and tuning strategies one method to overcome the frequency problem was to have the first two natural frequencies relatively close together. In addition to bending, torsion effects were taken advantage of to give a normalized power of $0.13561 \mathrm{Ws}^{4} \mathrm{~m}^{-2}$. The design, in Figure 50, depicts two masses placed asymmetrically on a beam at the tip, thus allowing torsion to be caused in the beam. [48]

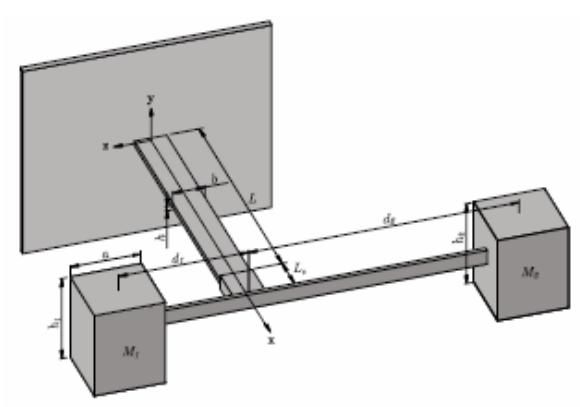

Figure 50 - Bending torsion harvester[48]

Another method of applying preload was through the use of magnets instead of directly applying them as Eichhorn did in his prototypes. A magnet was attached at the end of the beam while another magnet applied a repulsive force on it. By moving the magnet closer or further to the beam's magnet, one could change the axial stiffness and thus the natural frequency of the beam. This was validated through theory and experiments [49]. Figure 51 shows the magnetic axial tuning prototype. 


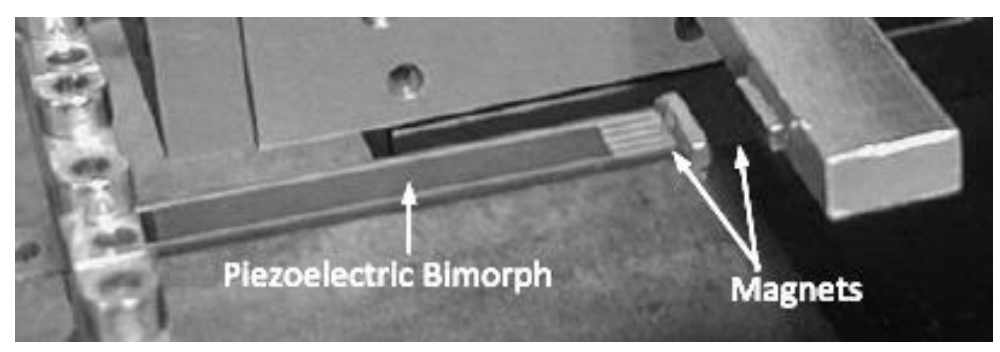

Figure 51 - Magnetic axial tuning bimorph [49]

\subsection{Methodology}

\subsubsection{Which Method?}

As mentioned above three methods of transduction exist for vibration harvesting: electrostatic, electromagnetic, and piezoelectric. The prior research shows that the piezoelectric method of transduction is the most promising of the three. From an energy density point of view according to Roundy and his research team, electrostatic devices had a power density of only $50 \mu \mathrm{W} / \mathrm{cm}^{3}$ compared to piezoelectrics which had a density of $250 \mu \mathrm{W} / \mathrm{cm}^{3}$ [2]. The literature also indicates that electrostatic devices require an additional voltage source whereas piezoelectric harvesters do not. The one advantage that electrostatic devices currently have over piezoelectric devices is their greater manufacturability according to current MEMS manufacturing technology [2]. For MEMS sized device, many researchers have commented on the inability of electromagnetic harvesters to produce a useful amount of power due to the spatial constraint limiting the amount of turns in a coil. Also the voltages generated were relatively small making it necessary to use a transformer to increase the voltage [2]. From the above discussion it can be concluded that piezoelectrics are a very promising transduction mechanism.

\subsubsection{Structure}

The literature review reveals that the simplest and most effective structure which best embodies the spring mass and damper system is the cantilever beam. The cantilever beam is simple yet effective. The reason why cantilevered beams are superior to other designs is due to the fact that the cantilevered design is able to produce more strain than other beam conditions when exposed to the same excitation, due to the fact that the cantilevered beam will have more deflection. For a piezoelectric harvester the choice between the 31 and 33 mode of operation is clear. As 
discovered by Lee et al. 31 is the mode of choice due to its superior poling compared to the 33 mode [39].

The only major drawback as mentioned previously is the inability of the cantilever beam to harvest appreciable amounts of power when the frequency of the ambient vibrations is significantly different from the natural frequency of the beam. The problem is best characterized by the researcher Jeon et al. in their paper when they wrote "The scavengeable power decreases by half if the applied frequency deviates $2 \%$ from the resonance frequency and almost goes away if the frequency deviation is more than 5\% from the resonant frequency" [31]. For the experiment it was decided that tuning of a cantilever device can overcome the frequency hurdle. 


\section{Chapter 3: Tuning Methods}

The following chapter will briefly review approaches to tuning and will determine that the application of the axial load is an effective method of tuning. Afterwards the mathematical theory of the effect an axial load has on the natural frequency of a cantilever beam will be documented. The results from the mathematical theory and results from finite element simulation are compared. It was found that a tensile load increases the natural frequency whilst compression decreased the natural frequency of a cantilever beam.

\subsection{Tuning Approaches for Vibration-Powered Power Harvesters}

To solve the natural frequency limitations of vibration power harvesters there are two separate approaches taken by researchers. The first approach is to create a structure or a design with various natural frequencies spaced close together on the spectrum thereby increasing the range of frequencies with which the harvester can operate. The second approach is to utilize a method to tune the natural frequency by changing a property of the device such as the effective stiffness or length.

The most common design is to create an array of beams of various lengths with the beams in each array having similar natural frequencies. The major disadvantage is that only one beam of the array is excited at resonance at a given point in time, resulting in poor energy density. Another method is to employ non-linear springs; these nonlinear springs are often composed of more complex geometries. Since the stiffness is non-linear, the natural frequency can change and track the excitation frequency. The advantage of these structures is that no extra power sources are required.

The second approach is to include a mechanism for frequency tuning. As detailed in the literature review, one possibility for frequency tuning is through the application of an axial force on a cantilever beam. The theory is detailed in the next section.

One mechanism that researchers have found successful involves applying an axial load to a beam via magnets. A magnet is attached to the end of a bimorph harvester and a separate magnet is used to either attract or repel the fixed magnet, causing a tensile or compressive force to be applied to the beam. The advantage of magnets is their additional use as a tip mass to target the beam to a specific frequency. Magnets also have minimal effect on beam structure. 
The disadvantage lies in the additional power consumption required to move the magnet for tuning. Also the forces applied to the magnet are not constant as the beam vibrates but tip displacements are generally small enough such that the axial force on the beam changes by negligible amounts [49].

\subsection{Effect of Axial Load on Natural Frequency of a Cantilever Beam}

Bokian investigated the effect of axial load on natural frequency of a cantilever beam [50]. Another study was published by NASA [51]. From their research equations were found for the relation of axial loading on the natural frequency of a cantilever beam in the form of Equation $3.1[51]$,

$$
\frac{\partial^{4} V}{\partial x^{4}}+\frac{P}{E I} \frac{\partial^{2} V(x, t)}{\partial x^{2}}+\frac{\rho}{E I} \frac{\partial^{2} V(x, t)}{\partial t^{2}}=0
$$

where $P$ is the applied axial load and $\mathrm{V}$ describes the deformation curve of the beam. A graph depicting the relation between axial force and resonance frequency can be seen in Figure 53. Tensile force and resonance frequency are found to have a positive linear relationship, whilst compressive force and resonance frequency have a negative non-linear relationship.

\subsubsection{Results: Theoretical versus Simulation}

The beam for which the relation between axial force and resonance frequency was plotted for is based on the piezoelectric harvesting beam which will also be used in the physical prototype manufactured and designed by Johnson Matthey (part number 427.0085.11Z). This beam has three layers with a central carbon fiber layer and a ceramic piezoelectric layer on both the top and the bottom. A schematic of the beam is included in Figure 52 and the beam dimensions plus material properties are recorded in Table 2.

The reason for choosing a carbon fiber material as the central layer is that it produces a beam which is less stiff; resulting in the natural frequency of the beam being lower than beams with a metal central layer. This is advantageous since the carbon fiber beam has a natural frequency of a couple hundred hertz, whereas a metallic beam of similar size would exhibit a natural frequency of a couple thousand hertz. Thus a significantly larger tipmass would be required to target the natural frequency of a metallic beam to frequencies of the ambient vibration. 


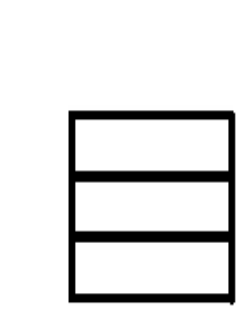

Front View

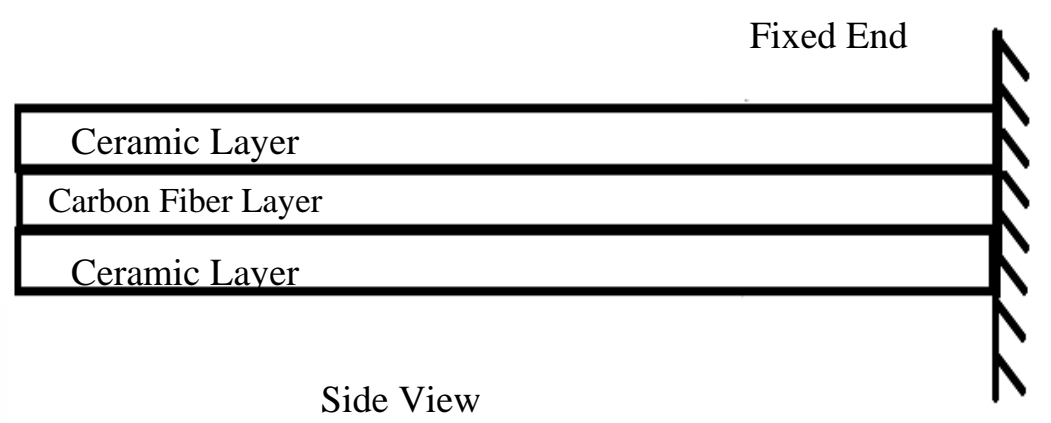

Figure 52- Layout of 427.0085.11Z beam [52]

Table 2- Dimensions of beam [52]

\begin{tabular}{ll}
\hline Length & $38.0+/-0.05 \mathrm{~mm}$ \\
\hline Width & $7.2+/-0.05 \mathrm{~mm}$ \\
\hline Thickness of Carbon Fiber Layer & $0.24+/-0.05 \mathrm{~mm}$ \\
\hline Thickness of Ceramic Layer & $0.27+/-0.03 \mathrm{~mm}$ \\
\hline Elastic Modulus of Ceramic & $4.85 \times 10^{10}$ \\
Elastic Modulus of Carbon Fiber & $2.00 \times 10^{10}$ \\
\hline
\end{tabular}

Since the Equation 3.1 describes solid beams of a single material, an equivalent stiffness term for the piezoelectric beam was calculated. The stiffness in Eq. 3.1 is represented by EI, which is calculated as the product between the elastic modulus and moment of inertia. The equivalent stiffness for the beam was found by using the parallel axis theorem to calculate the moment of inertia for each layer. The sum product of each layer's moment of inertia and corresponding elastic modulus supplied the equivalent stiffness for the piezoelectric beam.

$$
E I_{\text {eq }}=E I_{\text {top piezo }}+E I_{\text {carbon fiber }}+E I_{\text {bot piezo }}
$$

In addition the graph does not take the tip mass into account, and a closed form solution does not exist for a beam with a tip mass. 


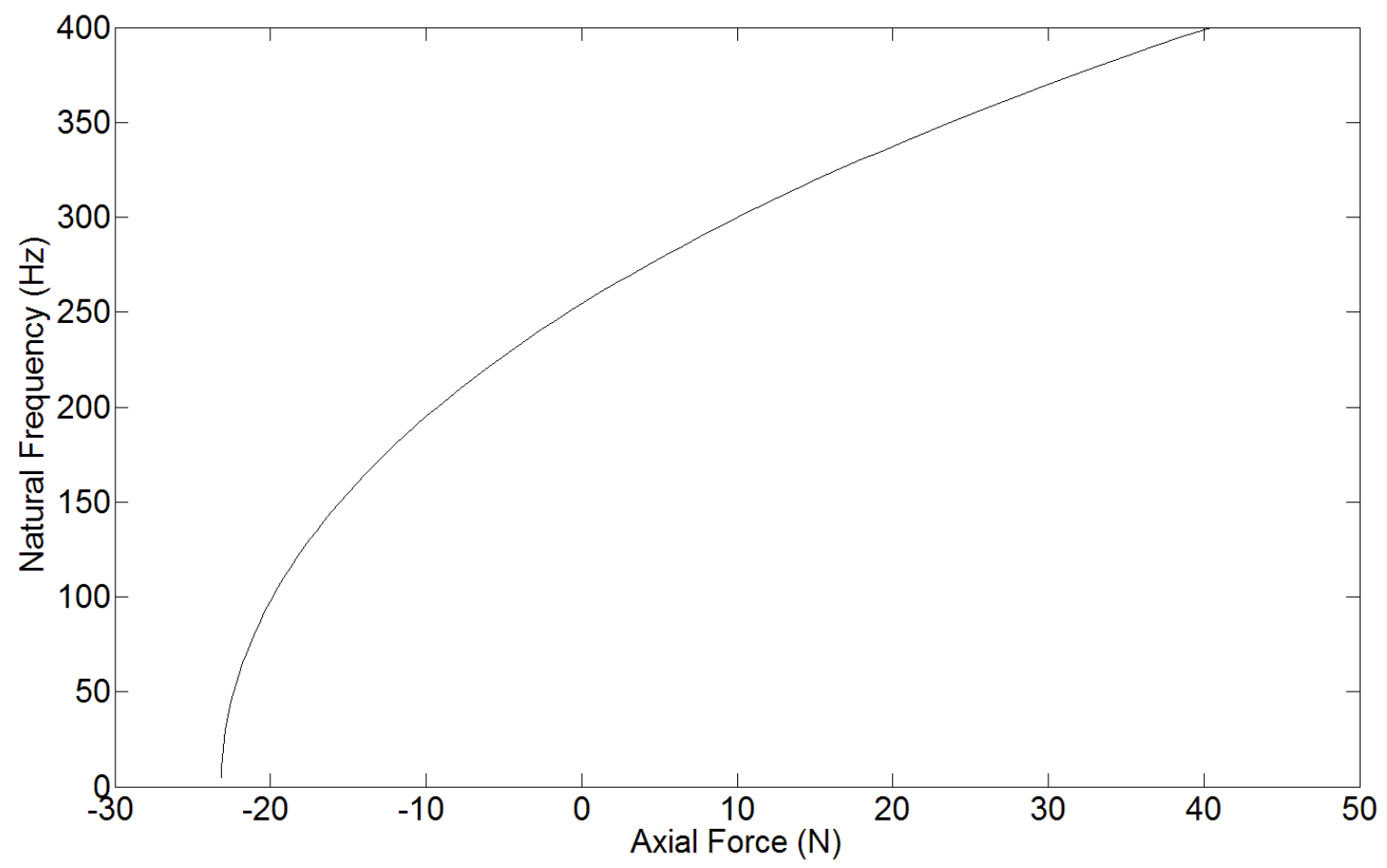

Figure 53 - Axial load vs. frequency graph based on theoretical equations

Using FEA methods, the effect of an axial load on a cantilever beam can be observed by taking harmonic sweeps. Harmonic analysis reveals that when a tensile load is applied, the resonance frequency increases with or without a tip mass. For the beam without a tip mass the natural frequency is at $315 \mathrm{~Hz}$ and increases to $330 \mathrm{~Hz}$ when a $4 \mathrm{~N}$ axial load is applied. The frequency increase is less drastic for a beam with a tip mass, which only increases from $101 \mathrm{~Hz}$ to $109 \mathrm{~Hz}$.

Slight discrepancies exist between the theoretical graph and FEA (ANSYS) results with frequencies from ANSYS being slightly lower as can be seen in Figure 54. The results from the finite element model are considered more accurate since numerous assumptions were used for the theoretical plot. The equation for the theoretical plot is for a beam with a same material throughout the beam, since this beam is a composite beam the equivalent stiffness was calculated to input into the stiffness term of the equation, the finite element model, takes into account the various layers of materials. As mentioned above, the stiffness for the beam was determined by calculating an equivalent stiffness for the composite material which may explain the frequency discrepancies. The linear pattern can be observed in the tension portion of the graph; however 
nonlinearity is less prominent in the compression results from ANSYS compared to the mathematical model.

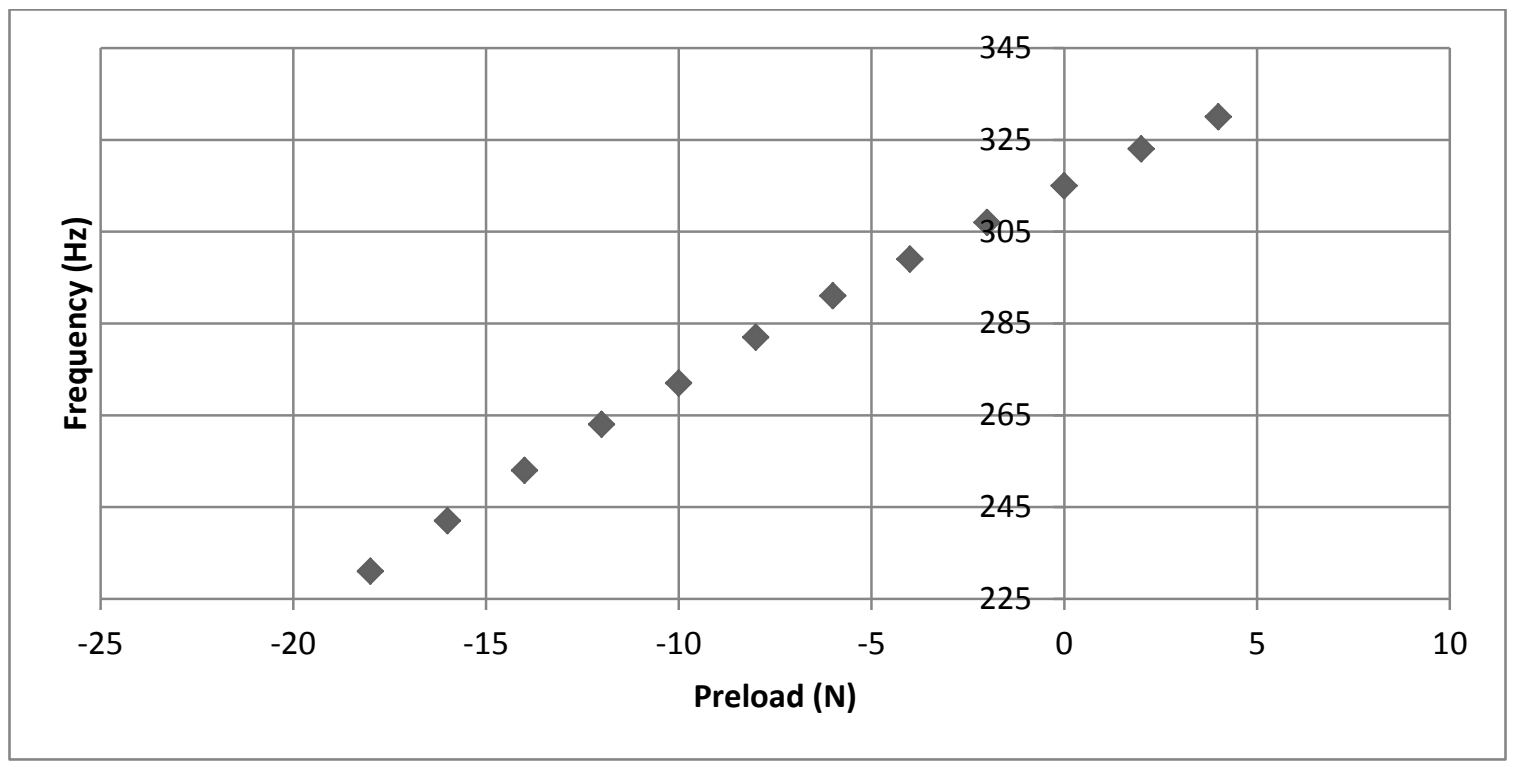

Figure 54 - Harmonic sweep of beam without tip mass ANSYS results

The above analysis does not take the tip mass into account since there is no closed form solution for a beam with a tip mass. Thus the graph for a beam with a tip mass is based solely on ANSYS results and is seen in Figure 56. The tip mass is a $7 \mathrm{~mm} \times 7 \mathrm{~mm} \times 5 \mathrm{~mm}$ N35 permanent magnet attached to the very tip of the same beam, this is illustrated in Figure 55.

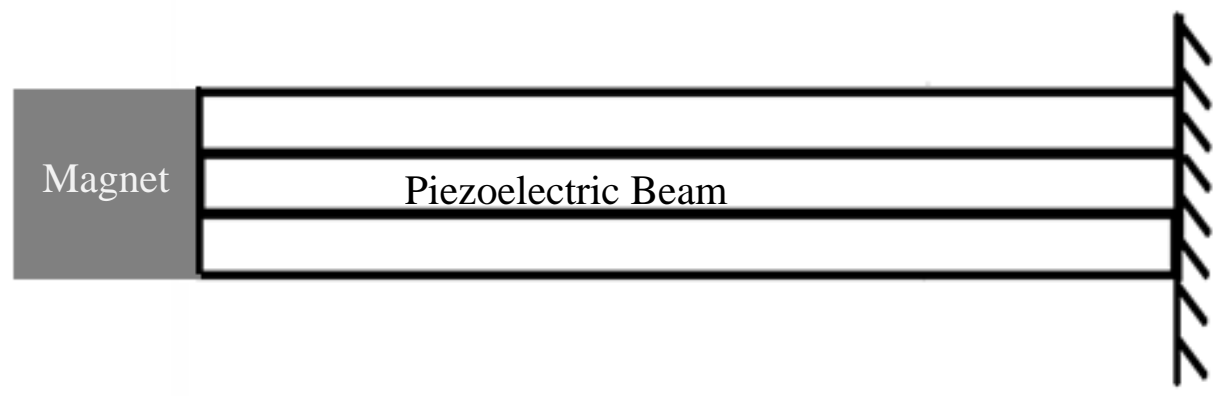

Figure 55 - Cantilever beam with tip mass

This was modeled and after running the FEA model to test the relation between axial force and natural frequency, a similar pattern was witnessed in the attached plot. Tension produces linear 
results while compression produced nonlinear results similar to the ANSYS model of the beam without mass.

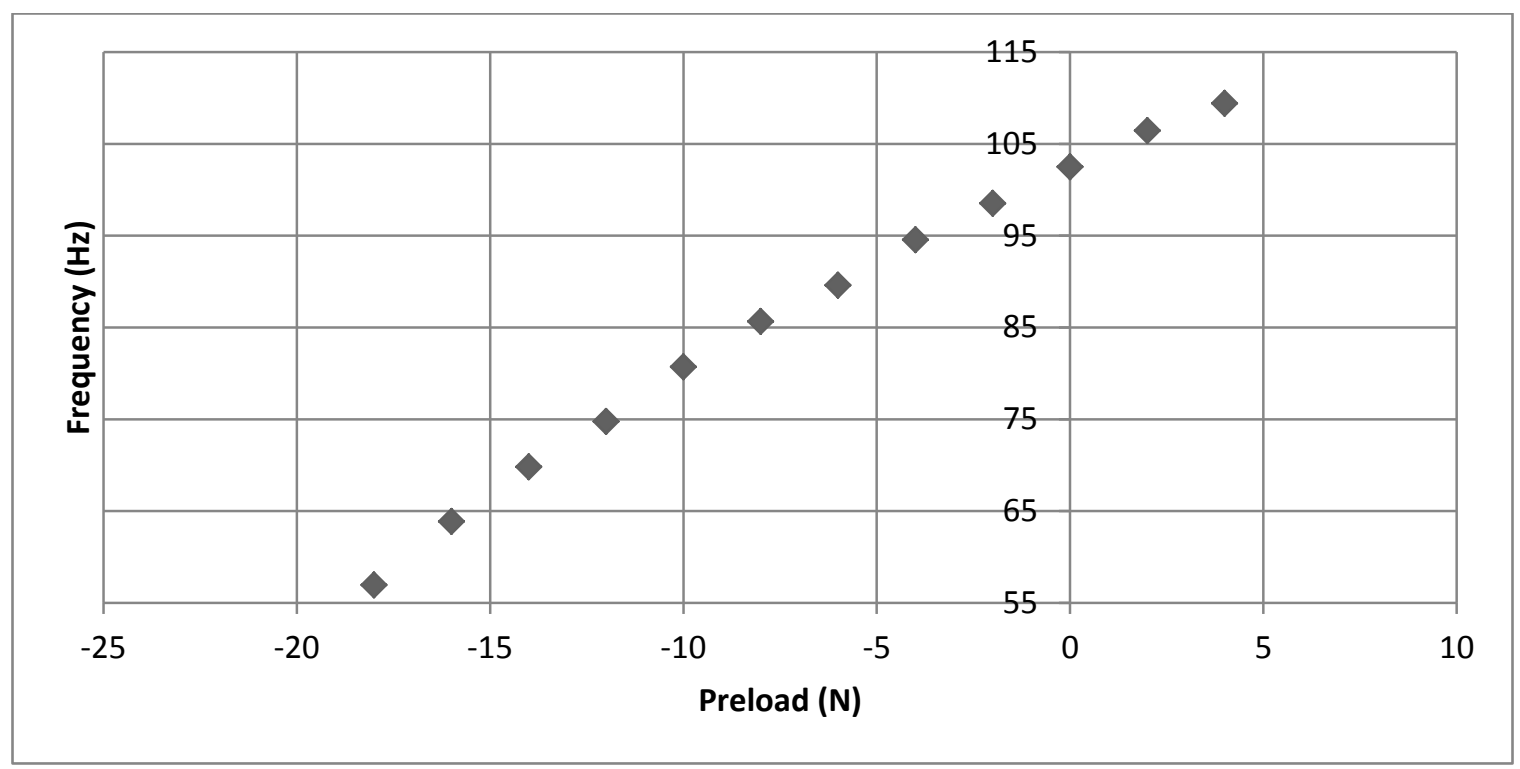

Figure 56 - Harmonic sweep of beam with tip mass ANSYS results 


\section{Chapter 4: Experimental Test}

The contents in this chapter are the design and of the physical prototype, which will use axial load tuning applied through permanent magnets. The relation of the distance between magnets and force applied is briefly examined. The experimental method and setup are then explained and finally the power results of tuning a beam subjected to shaker test are presented and it is seen that tuning does have a considerable benefit to the amount of power harvested. In this prototype an increase of more than seven times in power was observed.

\subsection{Power Harvesting Beam and Axial Load Adjustment Mechanism}

To verify the theory describing the effect of axial load on natural frequency of the cantilever beam, a prototype was constructed. The prototype was based on a paper by Waleed et al in 2011; in this paper the method of applying the axial load was to use the force between magnets [49]. By changing the distance between the two magnets the attractive or repulsive force could be changed. The distance adjustment would changes the magnetic force, which would subsequently change the natural frequency. One magnet was placed on the tip of the beam, whilst another was placed on an adjustment screw. The magnets were purchased from HKCM Magnetics. The completed testing rig is shown below in Figure 57.

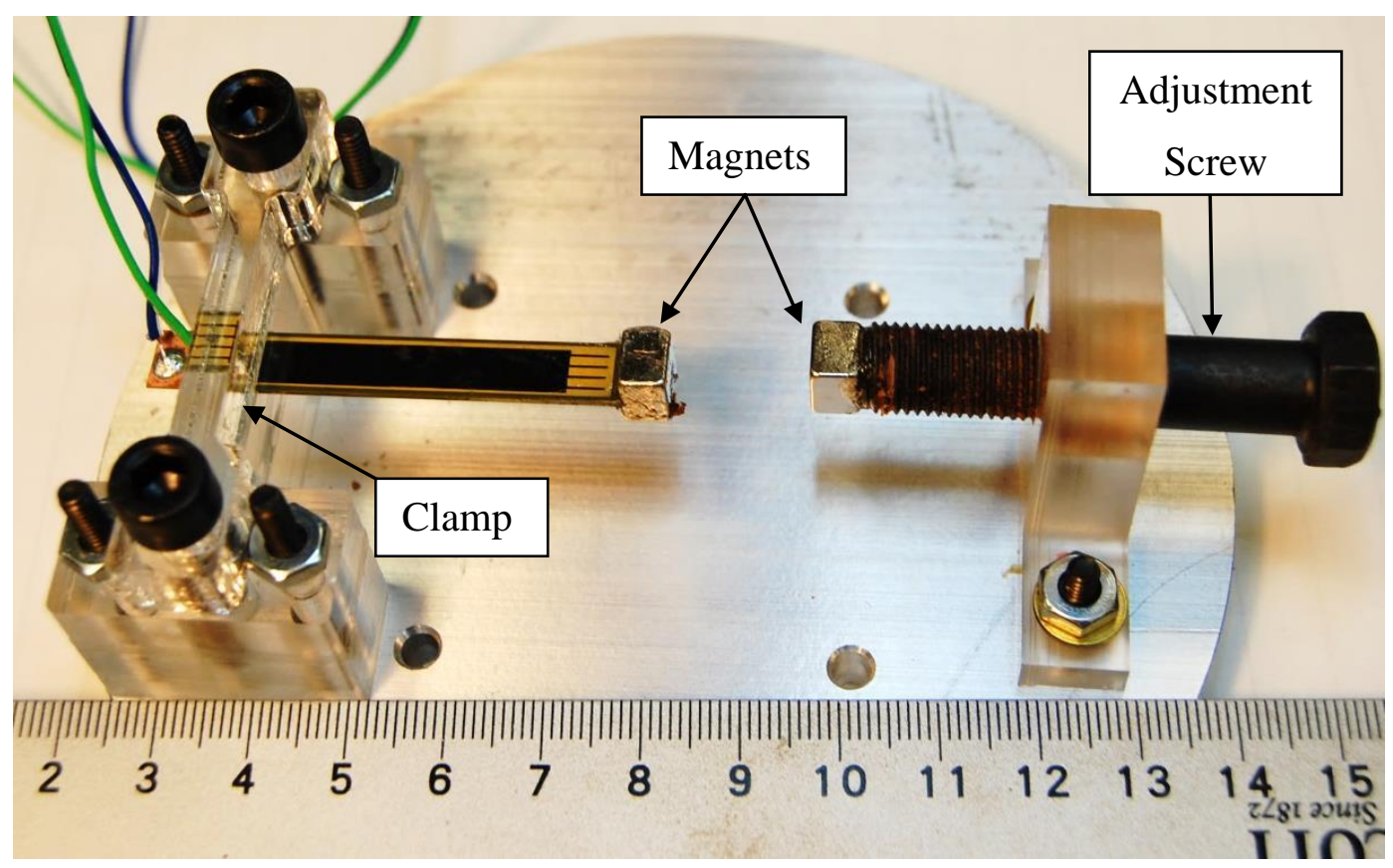

Figure 57- Completed testing rig 
The beam used was identical to the beam in Waleed's design. It was generously supplied by Johnson Matthey and is a triple layer beam with two VIBRIT 1100 ceramic piezoelectric layers sandwiching a central structural layer of carbon fiber. The beam had dimension of $49.95 \mathrm{~mm} \mathrm{x}$ $7.2 \mathrm{~mm} \times 0.78 \mathrm{~mm}$. Once fixed onto the experimental setup the beam free length was reduced to $38 \mathrm{~mm}$. The Figure 58 is a top view of the piezoelectric beam. The dimensions of the beam were mention in the previous chapter in Table 2.

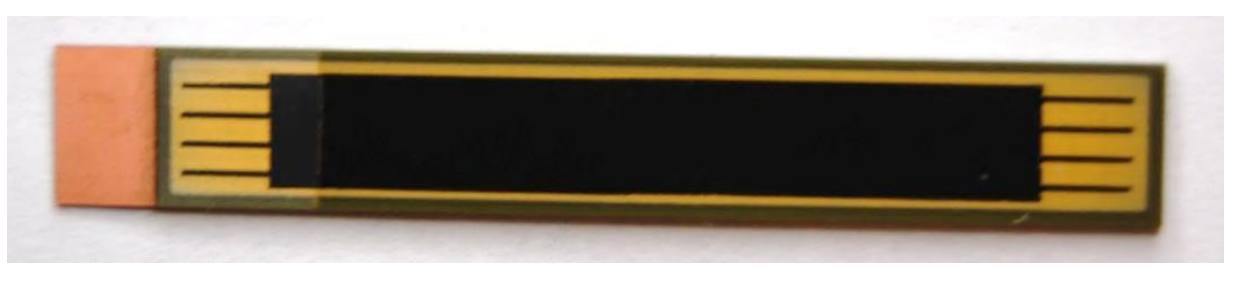

Figure 58 - Piezoelectric beam

The magnet is a Neodymium N35 magnet, with dimensions of $7 \mathrm{~mm} \times 7 \mathrm{~mm} \times 5 \mathrm{~mm}$ purchased from HKCM Engineering (Q07x07x05Ni-N35). The surface area between the two magnets was $7 \mathrm{~mm}$ by $7 \mathrm{~mm}$. Since the axial force is dependent on the distance between the two magnets, the relation can be determined. However the calculations are complicated. One research paper used an online calculator provided by the company K\&J Magnetics to determine the relation. From that relation the researchers created a coefficient to fit the existing equation to the data [49]. It is possible to use finite elements to investigate this as well; the same finite element software ANSYS was used. The Figure 59 shows the relation of the force between two magnets at various distances. The results are from the software, online calculator and measured from bottom face of one magnet to the top face of the opposite.

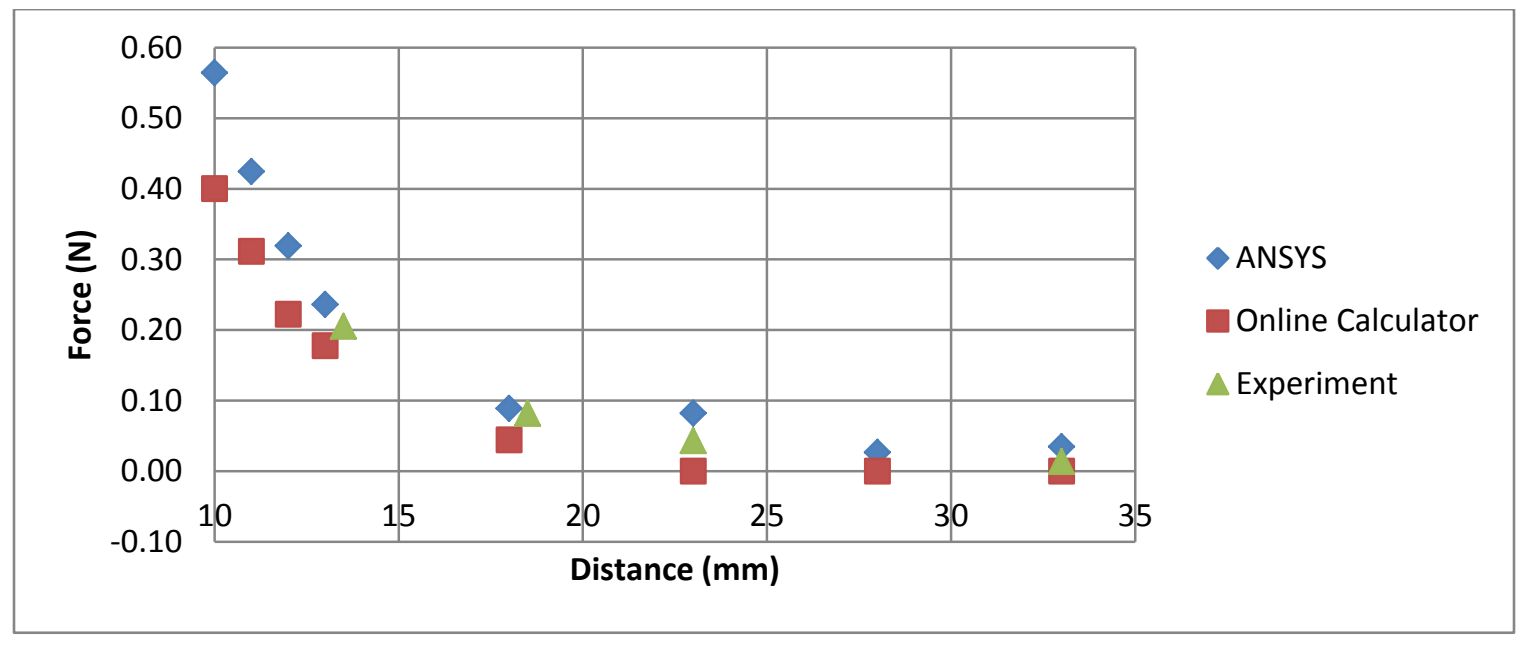


Figure 59- Relation between magnetic force and distance when one magnet is placed on adjustment screw

A simple experiment was set up to measure the force of the magnet, a photograph of the setup is attached, Figure 60. A digital scale was used and a test rig to hold a magnet at various distances was erected above the scale. One magnet was glued onto the plate of the scale whilst the other was glued to the test rig. Various distances were tested and the reading on the digital scale was recorded.

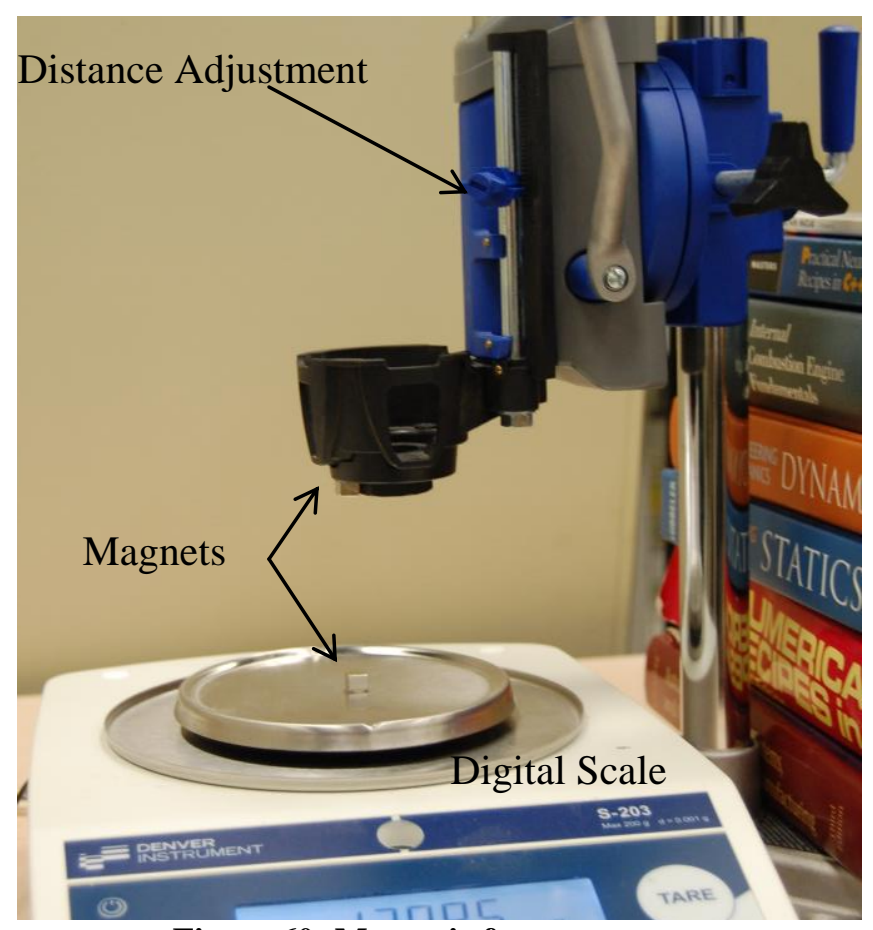

Figure 60 -Magnetic force test setup

However with only one magnet, the maximum force of the setup that could reach is only $0.21 \mathrm{~N}$. In order to increase the magnitude of force applied, two magnets are placed on the adjustment screw to produce a maximum of $0.94 \mathrm{~N}$, this can be seen in Figure 61. The photograph below, Figure 62, shows two magnets on the adjustment screw. 


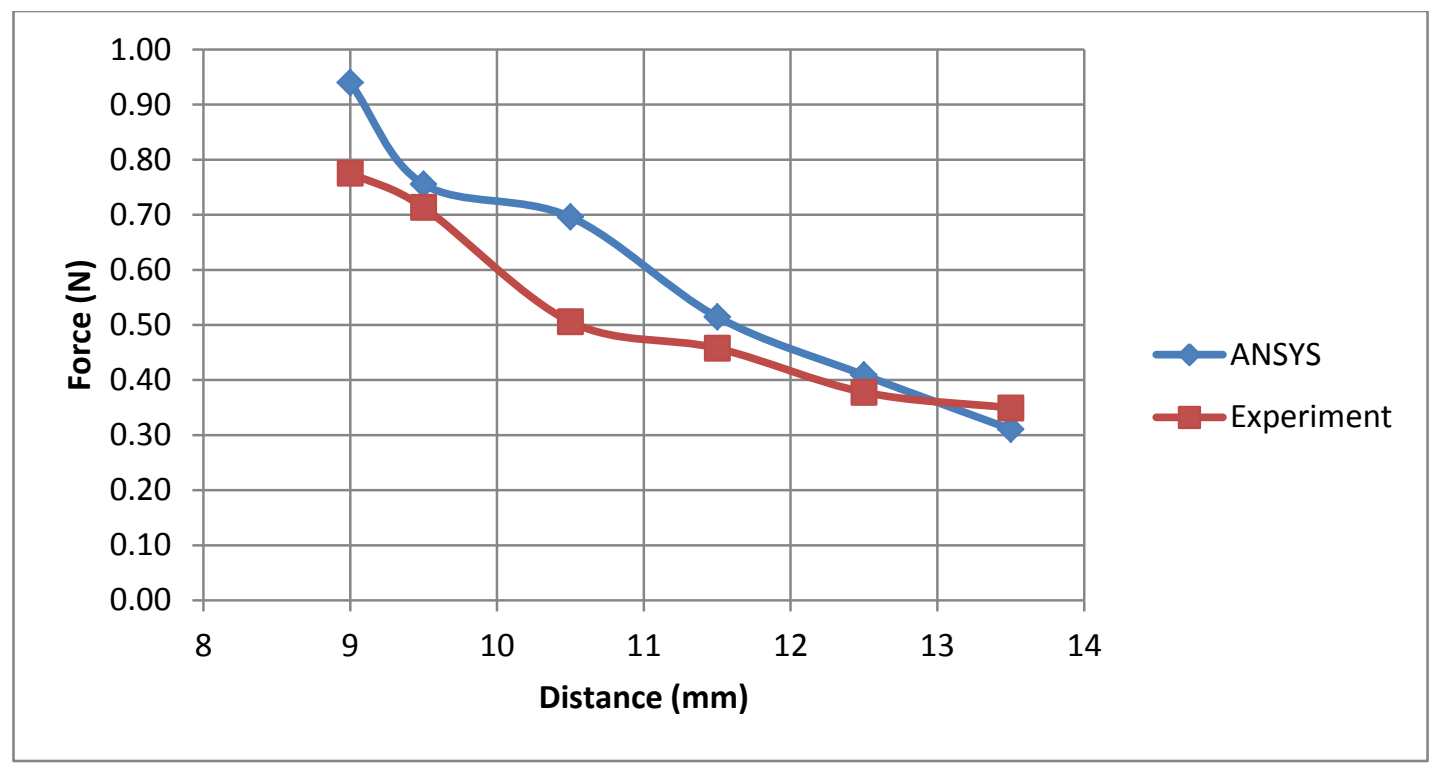

Figure 61 -Relation between magnetic force and distance when two magnets are placed on adjustment screw

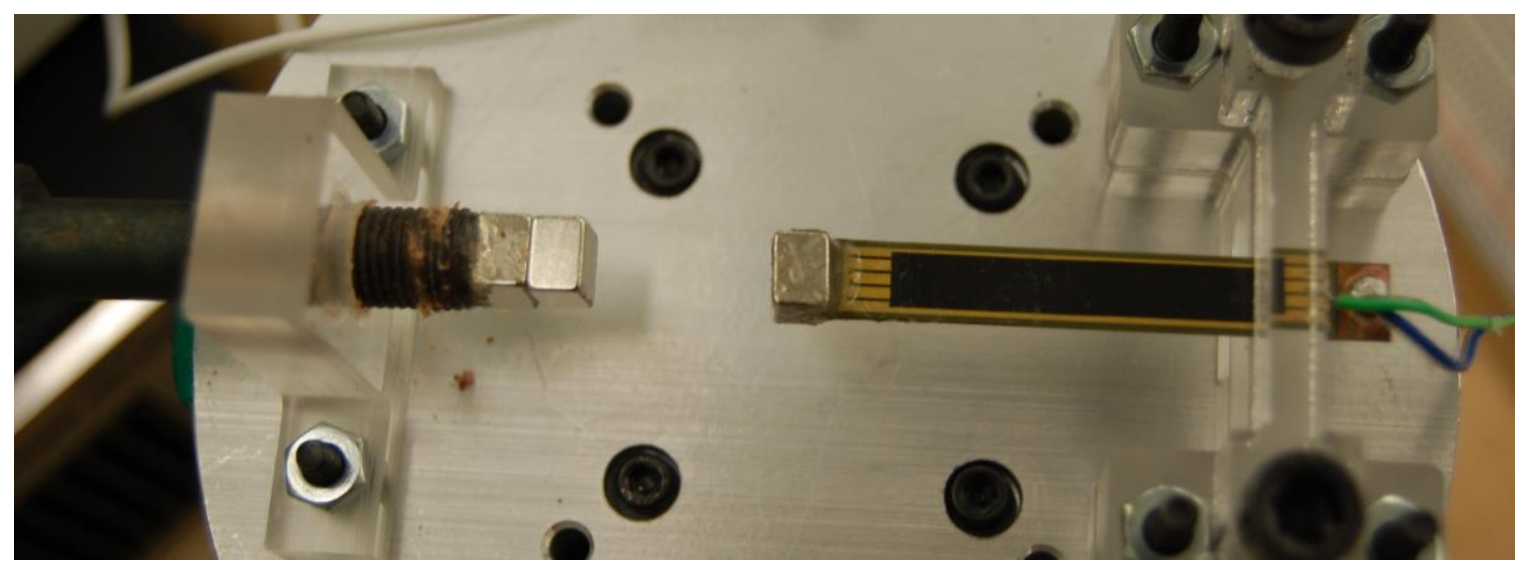

Figure 62 -Two magnets on adjustment screw

Theoretically, force could be further increased, for example, three magnets would produce a maximum of $3.78 \mathrm{~N}$ seen in Figure 63, but practically only $1.2 \mathrm{~N}$ could be applied reliably. The photograph, Figure 64, shows the setup with three magnets present on the adjustment screw. 


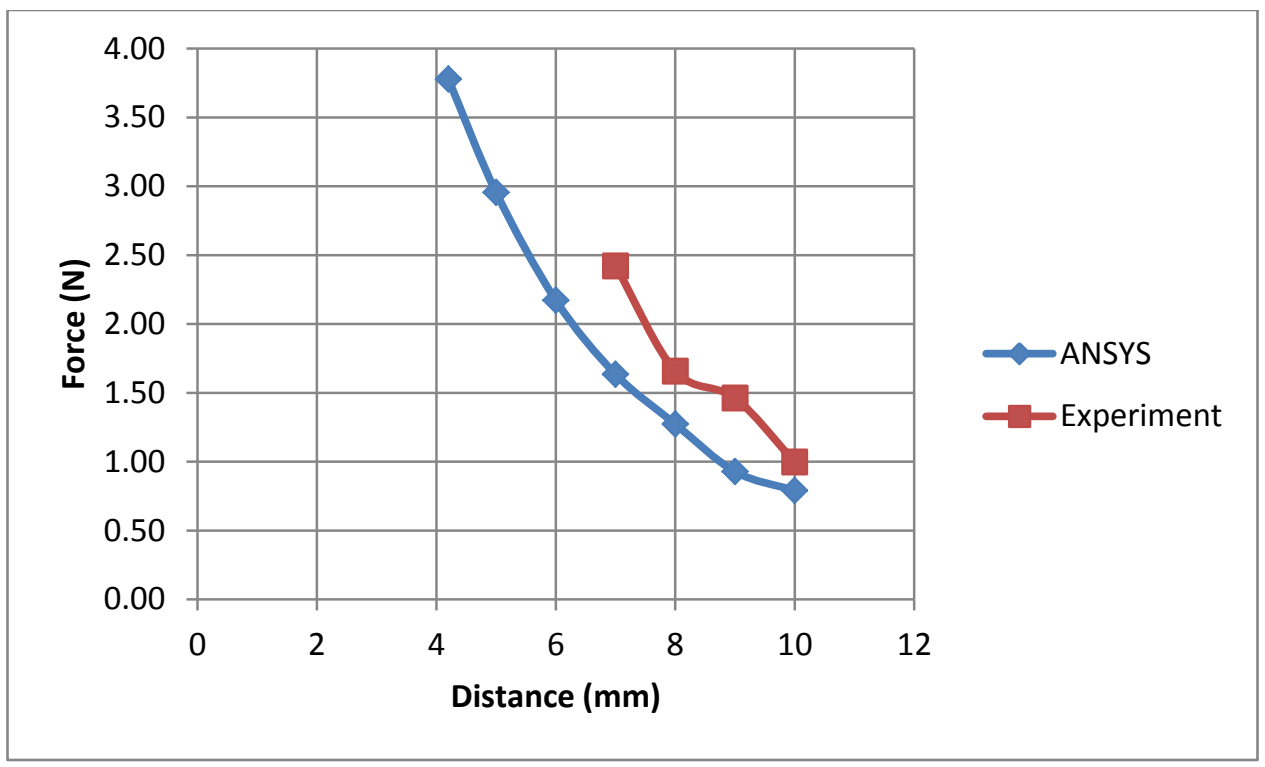

Figure 63 -Relation between magnetic force and distance when three magnets are placed on adjustment screw

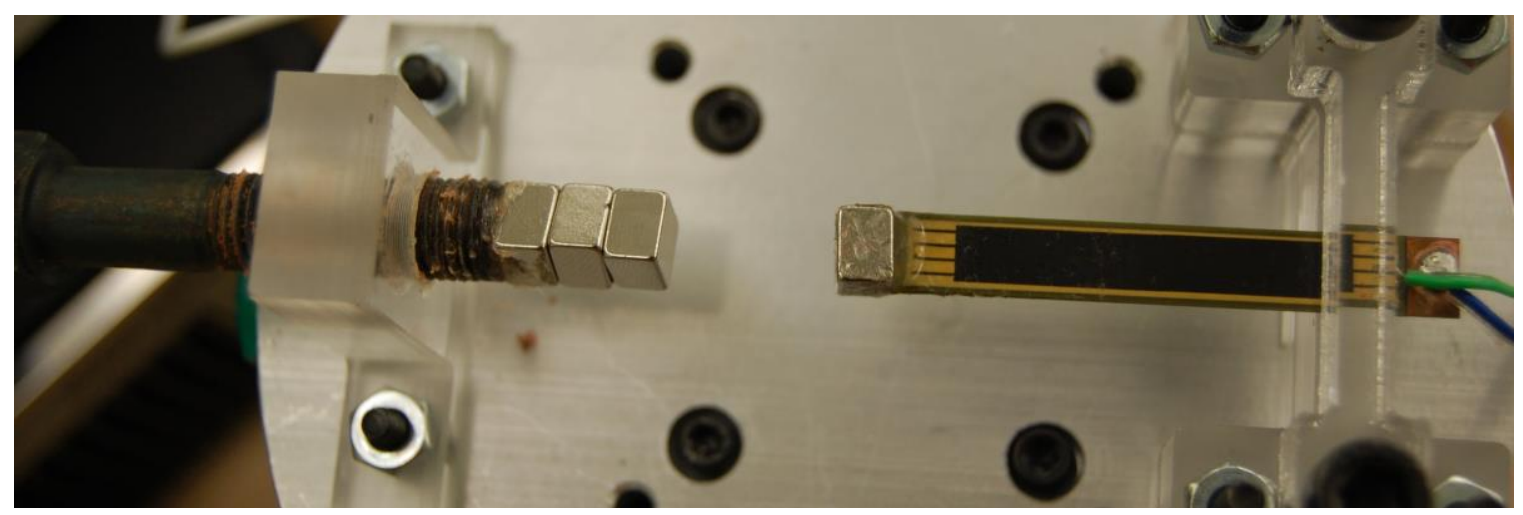

Figure 64 - Three magnets on adjustment screw

The base of the test rig was a big circular aluminum plate as showing in Figure 65. Figure 65 is a rendering of the setup which would be placed on the shaker. On this plate the tuning mechanism was be installed. A clamp for the piezoelectric beam with a magnet on the tip was placed on one end of the plate while directly opposing it an adjustment screw with a magnet at the end was placed on a holder. The screw moved the magnet closer or further from the magnet on the beam 


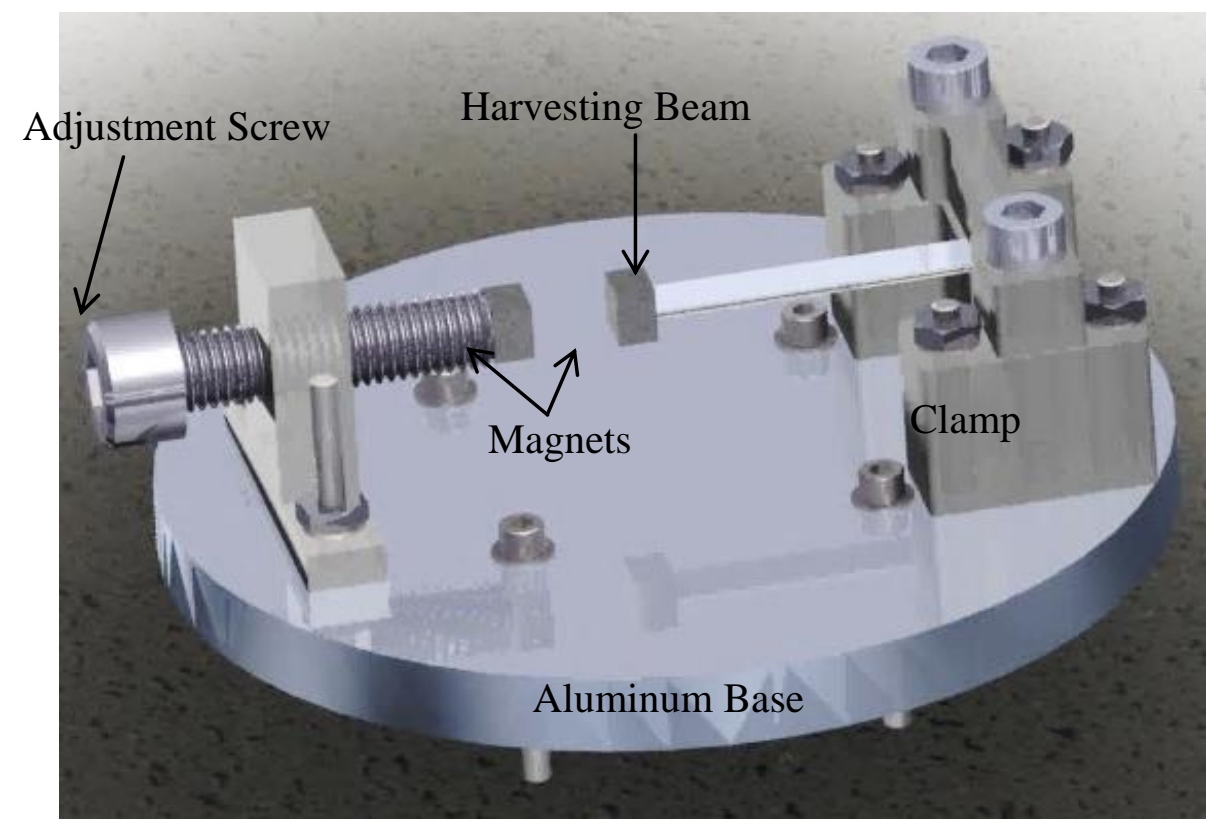

Figure 65 - Rendering of experimental setup for shaker

The assembly of the prototype was straightforward with most parts attached to the aluminum plate with 6-32 socket head cap screws of varying lengths. The 6-32 screw size was chosen since it was the screw size required for the attachment to the shaker. The magnets were attached to the end of the beam and to end of the screw with an epoxy adhesive (Loctite E-30CL). This adhesive is suitable due to its strong adherence to both metals and ceramics. According to Marks Standard Handbook for Mechanical Engineers, Epoxy resin has a rating of 8 out of 10 for both ceramics and metals whereby the larger the stronger[53]. To assemble the epoxy adhesive the beam and the magnet were held in position with tape for over four hours to give the epoxy enough time to cure. For the electrical connection to the beam two wires were attached by soldering one wire to the copper pad and another wire to the top of the piezoelectric beam. Resistors were connected across the two wires with a solderless breadboard. A circuit diagram is attached as Figure 66.

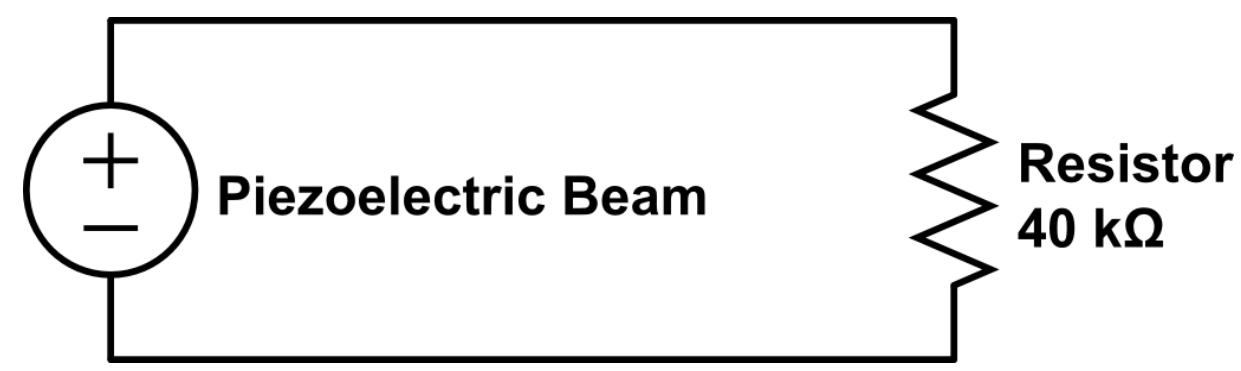

Figure 66 -Electric circuit of experimental setup 


\subsection{Experimental Setup}

The experiments tests were on an ET-126B Electrodynamic Shaker manufactured by Labworks Inc. The prototype was tested with frequencies ranging from $75 \mathrm{~Hz}$ to $100 \mathrm{~Hz}$ with a constant input displacement of $2 \mathrm{E}-5 \mathrm{~m}$. The magnets were also separated at various distances apart from each other, representing various axial load conditions. The main goal of the experiment was to demonstrate that the natural frequency of the beam would change as the distance between the magnets were changed, affecting the axial load applied. The axial load can be changed from tensile to compressive by flipping the poles of the magnet on the adjustment screw. Using a single magnet, a maximum tensile force of $0.2 \mathrm{~N}$ was achieved. By attaching another magnet to the end of the screw a maximum of $0.9 \mathrm{~N}$ was reached, with three magnets a tensile force of $3 \mathrm{~N}$ can be reached. However in compression mode $1.2 \mathrm{~N}$ is the maximum that can be applied reliably as the beam has a tendency to flip upwards and be close to snapping or buckling if the force is increased furthermore. An accelerometer is attached to the aluminum plate via a threaded hole. Shown below in Figure 67 is a schematic of the connections of the experimental setup.

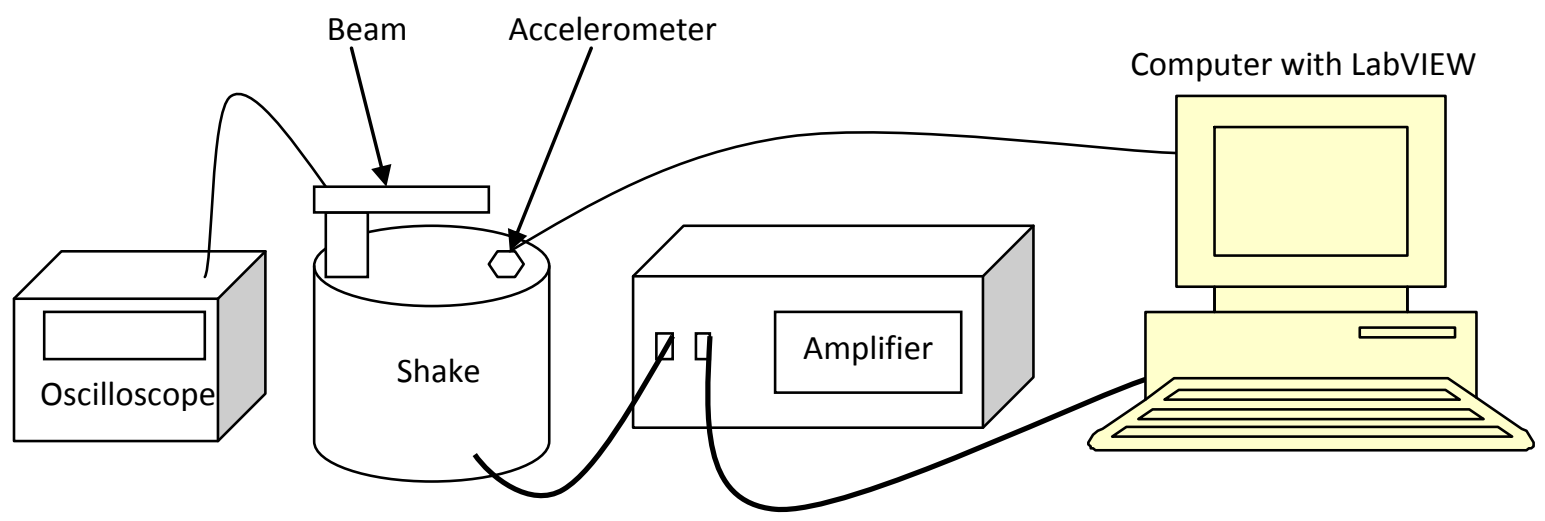

Figure 67 - Schematic of experimental setup connections

The of the shaker was controlled by the power amplifier manufactured by Labworks Inc., with the model number pa-138. The signal from the accelerometer was sent to the data acquisition card (DAQ) in the computer and recorded in the LabVIEW software. To measure the voltage from the piezoelectric beam a digital storage oscilloscope manufactured by Agilent technologies was used, model DS03102A. The LabVIEW software sent a signal to the amplifier. From the amplifier the signal for the base excitation was sent to the shaker. The piezoelectric beam was connected to the oscilloscope to read the voltage output. A photograph of the setup is attached as Figure 68 . 


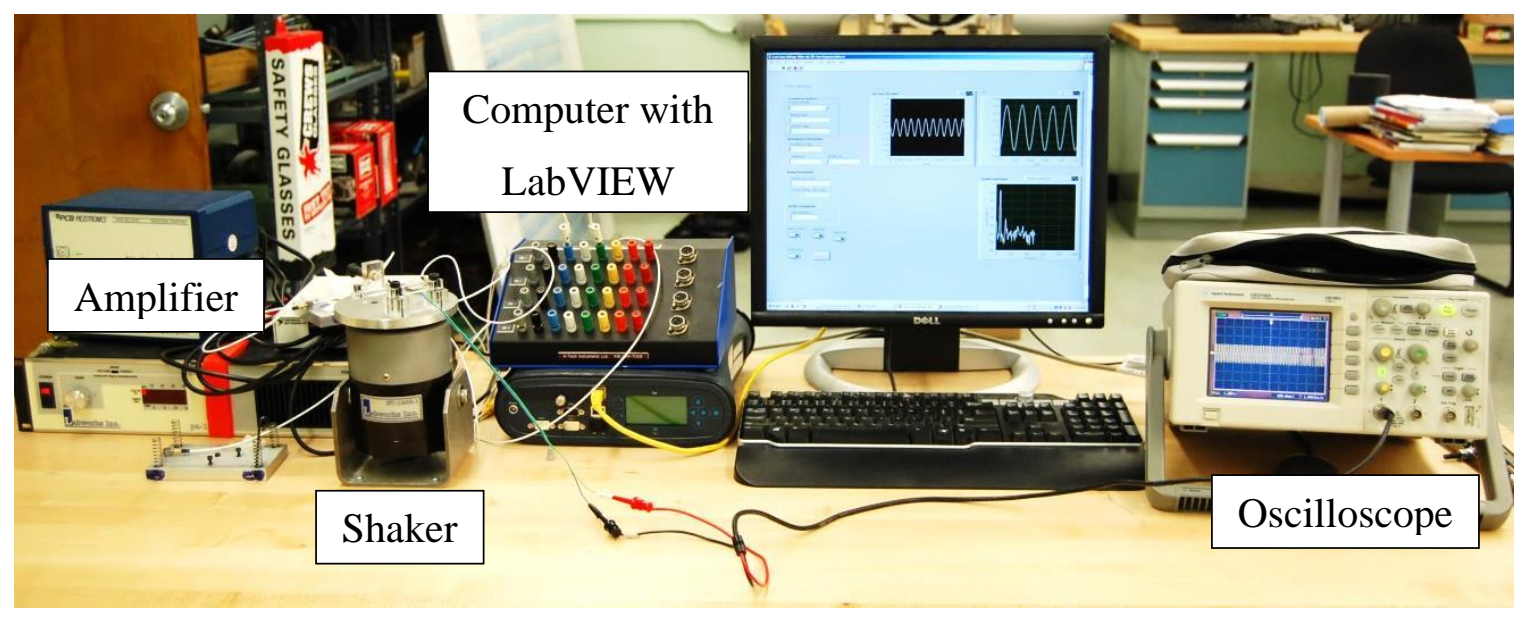

Figure 68 - Experimental setup

\subsection{Experimental Results}

From the experiment, the first test run was to determine the open circuit's natural frequency with a frequency sweep. When no axial load was applied it was found to be $82 \mathrm{~Hz}$. When the maximum tensile load of $0.94 \mathrm{~N}$ was applied the natural frequency was found to be $93 \mathrm{~Hz}$. Thus the tunable range of the prototype in tension operation, as seen in Figure 69, was from $82 \mathrm{~Hz}-93$ $\mathrm{Hz}$ when the preload range was 0 to $0.94 \mathrm{~N}$. In compression mode, as seen in Figure 70 , the frequency at the maximum load of $1.20 \mathrm{~N}$ was $67 \mathrm{~Hz}$, thus the range for the compression mode was $67 \mathrm{~Hz}$ to $82 \mathrm{~Hz}$. Figure 71 is both the tension and compression plotted on a single plot, with the negative representing compression and positive representing tension. 


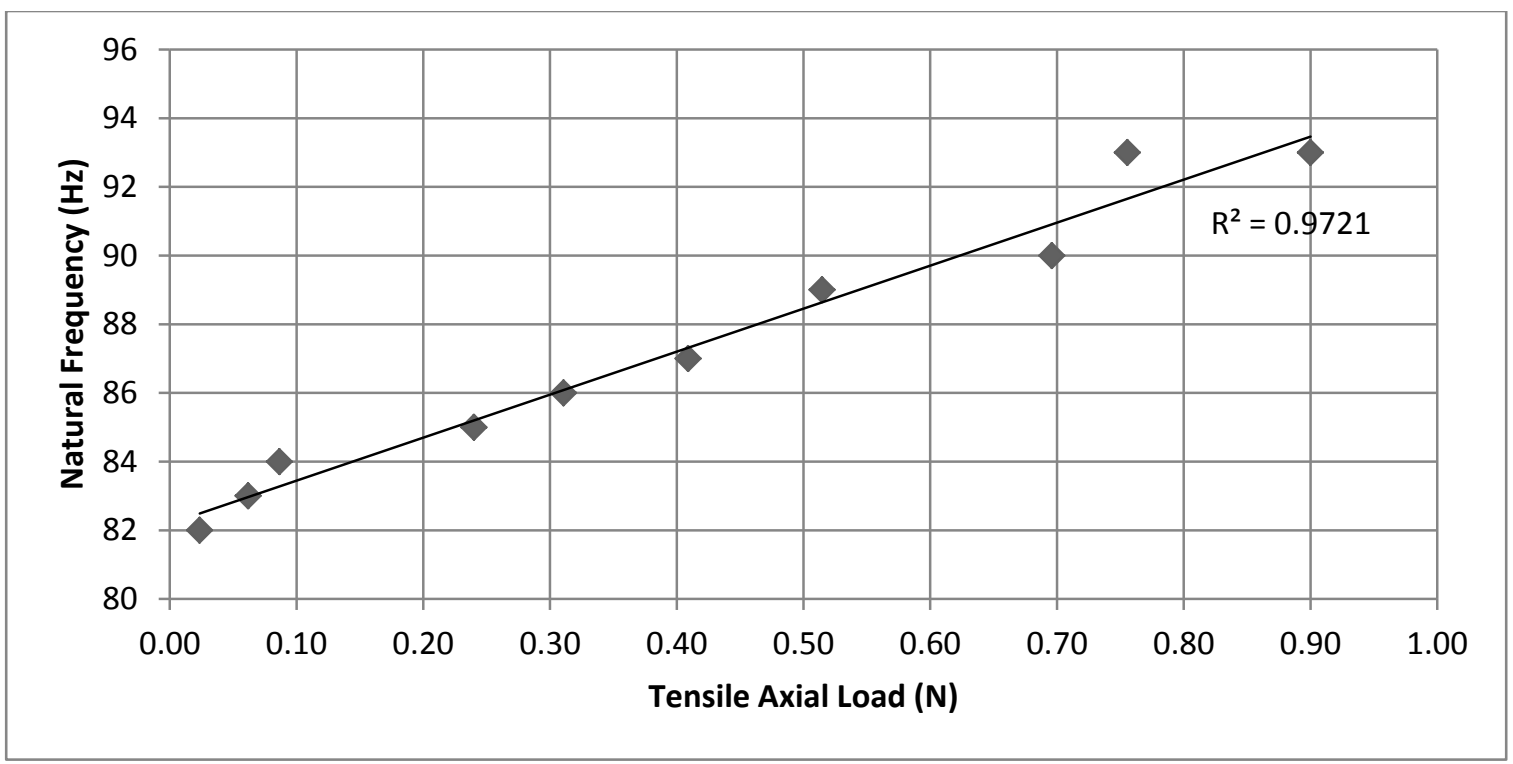

Figure 69 - Relation between the natural frequency and the tension preload

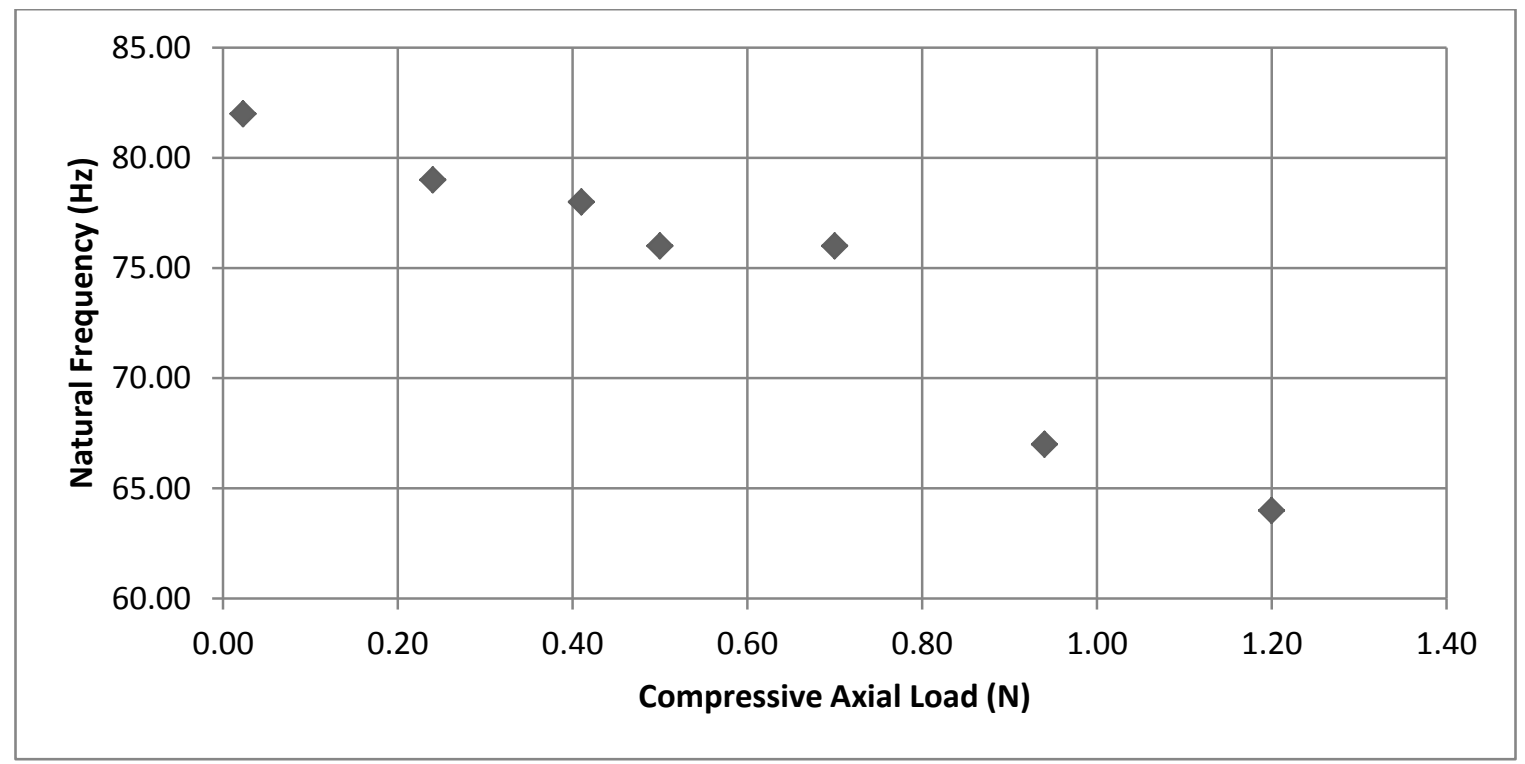

Figure 70 - Relation between the natural frequency and the compression preload 


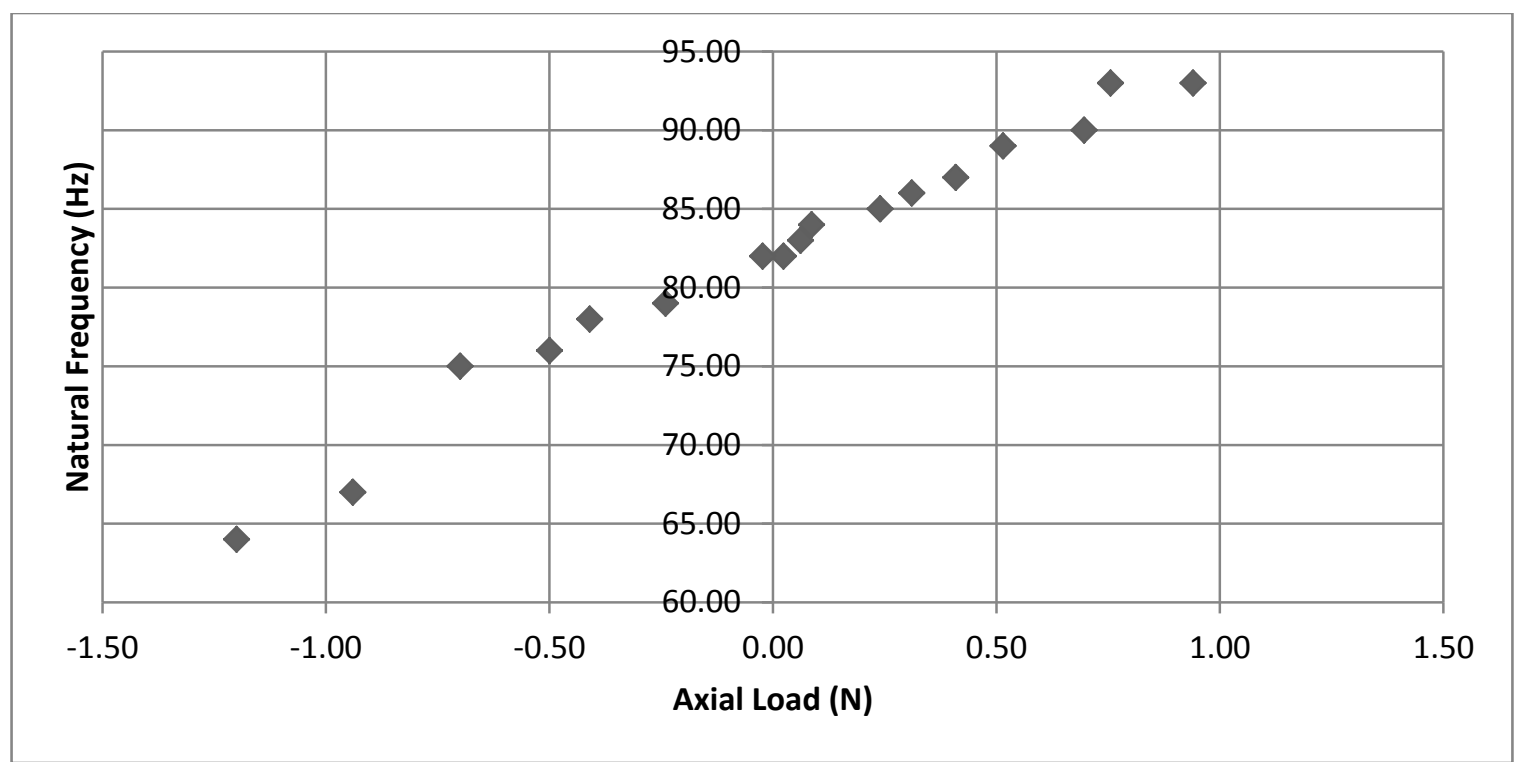

Figure 71- Relation between the natural frequency and both compression and tension preload

As expected when a resistor load is connected to the circuit, the natural frequency of the system decreases since it acts as a damper and removes energy from the system. The natural frequency decreased approximately $3 \mathrm{~Hz}$ in some of the tests which is well within the range reported by previous papers.

The plots show a linear relationship between the resonance frequency and axial load in tension as predicted by the pattern in the theory. A linear trendline with an R squared value of 0.9721 was added to further illustrate this fact. The compression portion still appeared to be linear. The amount of compression force applied was not sufficient to show the nonlinear relation between the load and the frequency as predicted by the theory. The amount of force could not be increased above $1.2 \mathrm{~N}$ in compression, as the beam kept flipping upwards when the magnets repelled each other.

From the experiment the optimal resistance was either $40 \mathrm{k} \Omega$ or $50 \mathrm{k} \Omega$, it can be seen from Table 3 and Table 4 that thirteen load conditions have an optimal resistor value of $40 \mathrm{k} \Omega$ and four of the load conditions have $50 \mathrm{k} \Omega$. The optimal resistor value is the resistance applied which allows this device to harvest the greatest amount of power, and this resistance value was found from experimental testing. 
The maximum power output had a minimal range from $0.587 \mathrm{~mW}$ to $0.402 \mathrm{~mW}$. There appears to be no discernible pattern for this case. It is believed that the discrepancy could be because adjusting the knob on the amplifier; one cannot reach the same exact displacement consistently. As the experiments were conducted over several days, experiments on different days may have had small variations in input although utmost care was taken in keeping conditions consistent.

Table 3 - Power output of tensile mode

\begin{tabular}{cccc}
\hline Force $(\mathbf{N})$ & Natural Frequency $(\mathbf{H z})$ & Max. Power $(\mathbf{W})$ & Optimal Resistance $(\mathbf{k} \Omega)$ \\
\hline $\mathbf{0 . 9 0}$ & 93 & $4.59 \mathrm{E}-04$ & 40 \\
$\mathbf{0 . 7 6}$ & 93 & $4.25 \mathrm{E}-04$ & 40 \\
$\mathbf{0 . 7 0}$ & 90 & $4.38 \mathrm{E}-04$ & 40 \\
$\mathbf{0 . 5 1}$ & 89 & $4.80 \mathrm{E}-04$ & 50 \\
$\mathbf{0 . 4 1}$ & 87 & $5.00 \mathrm{E}-04$ & 40 \\
$\mathbf{0 . 3 1}$ & 86 & $5.27 \mathrm{E}-04$ & 50 \\
$\mathbf{0 . 2 4}$ & 85 & $4.02 \mathrm{E}-04$ & 50 \\
$\mathbf{0 . 0 9}$ & 84 & $4.27 \mathrm{E}-04$ & 40 \\
$\mathbf{0 . 0 6}$ & 83 & $4.24 \mathrm{E}-04$ & 50 \\
$\mathbf{0 . 0 2}$ & 82 & $4.29 \mathrm{E}-04$ & 40 \\
\hline
\end{tabular}

Table 4- Power output of compression mode

\begin{tabular}{cccc}
\hline Force $(\mathbf{N})$ & Natural Frequency $(\mathbf{H z})$ & Max. Power $(\mathbf{W})$ & Optimal Resistance $(\mathbf{k} \Omega)$ \\
\hline $\mathbf{0 . 0 2}$ & 82 & $5.87 \mathrm{E}-04$ & 40 \\
$\mathbf{0 . 2 4}$ & 79 & $5.62 \mathrm{E}-04$ & 40 \\
$\mathbf{0 . 4 1}$ & 78 & $5.40 \mathrm{E}-04$ & 40 \\
$\mathbf{0 . 5 0}$ & 76 & $5.36 \mathrm{E}-04$ & 40 \\
$\mathbf{0 . 7 0}$ & 76 & $5.15 \mathrm{E}-04$ & 40 \\
$\mathbf{0 . 9 4}$ & 67 & $5.28 \mathrm{E}-04$ & 40 \\
$\mathbf{1 . 2 0}$ & 64 & $4.89 \mathrm{E}-04$ & 40 \\
\hline
\end{tabular}

To further illustrate the tuning's effectiveness, if no axial load is applied; the $0.02 \mathrm{~N}$ case is almost equivalent to $0 \mathrm{~N}$. A beam without tuning has a natural frequency of 83 Hz. Several excitation frequencies are examined $(86 \mathrm{~Hz}, 90 \mathrm{~Hz}$ and $93 \mathrm{~Hz})$ and the tuned power is compared to the power of a beam with no axial load. Table 5 and bar chart, Figure 72, below show that tuning allows significantly more power to be harvested as the difference between the excitation frequency and natural frequency of the untuned 
beam increases. In the most extreme case, the prototype is able to increase the power harvested by more than seven times when tuned at $93 \mathrm{~Hz}$.

Table 5- Table showing effectiveness of tuning

\begin{tabular}{lcccc}
\hline Frequency (Hz) & $\mathbf{7 6 ~ H z}$ & $\mathbf{8 6 ~ H z}$ & $\mathbf{9 0 ~} \mathbf{~ H z}$ & $\mathbf{9 3 ~ H z}$ \\
\hline Untuned Power (W) & $1.38 \mathrm{E}-04$ & $1.66 \mathrm{E}-04$ & $8.91 \mathrm{E}-05$ & $5.36 \mathrm{E}-05$ \\
Tuned Power (W) & $5.15 \mathrm{E}-04$ & $5.27 \mathrm{E}-04$ & $4.38 \mathrm{E}-04$ & $4.59 \mathrm{E}-04$ \\
Difference (W) & $3.77 \mathrm{E}-04$ & $3.60 \mathrm{E}-04$ & $3.49 \mathrm{E}-04$ & $4.05 \mathrm{E}-04$ \\
\% Power Increase & $273 \%$ & $217 \%$ & $392 \%$ & $756 \%$ \\
\hline
\end{tabular}

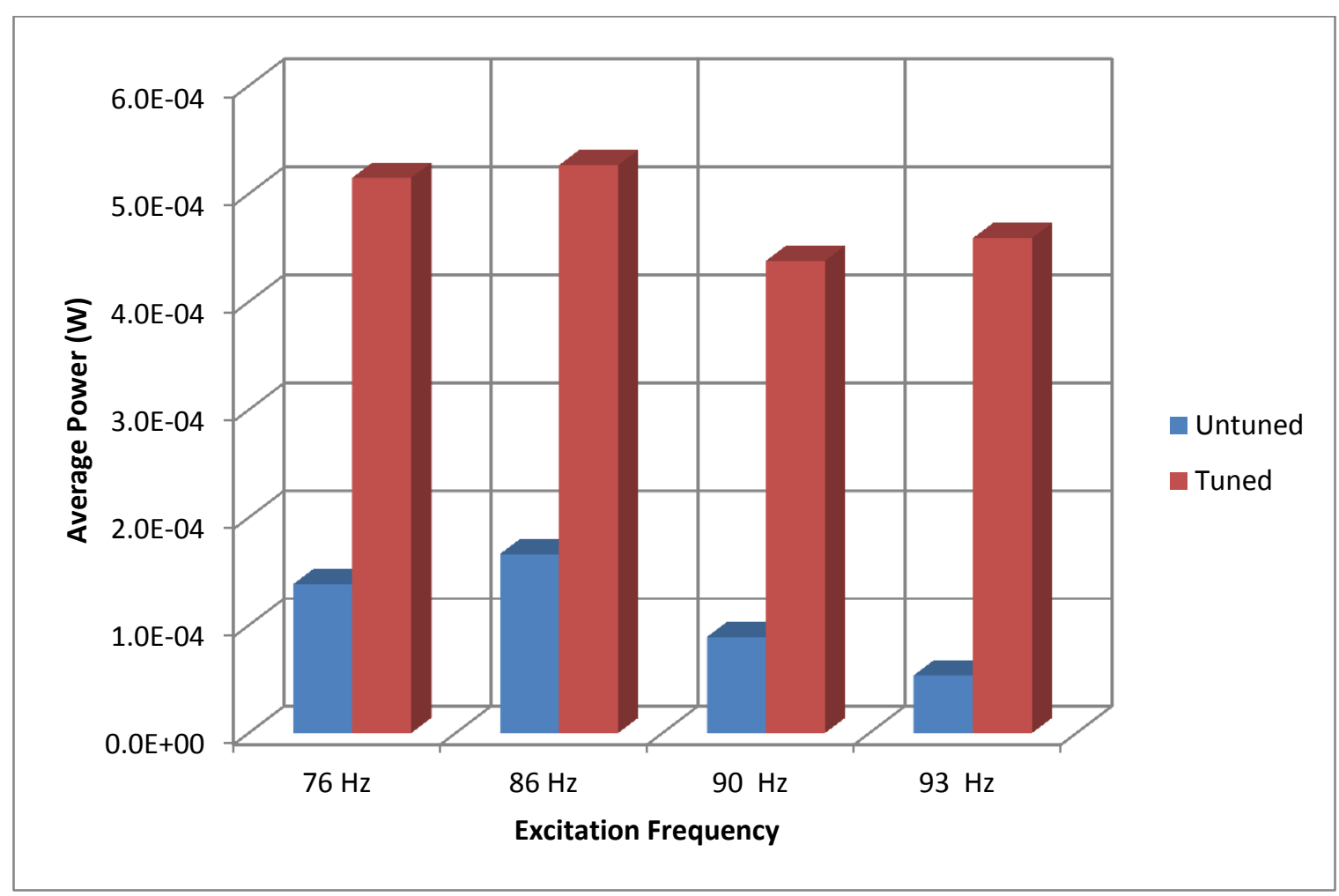

Figure 72- Comparison between tuned and untuned cases

To illustrate the tuning capabilities, the frequency sweep and power outputs at $40 \mathrm{k} \Omega$ of resistance of different axial load cases are plotted in Figure 73 and Figure 74. From the graphs the increased frequency range can be seen, in addition to the power of the beam under various axial load values. 


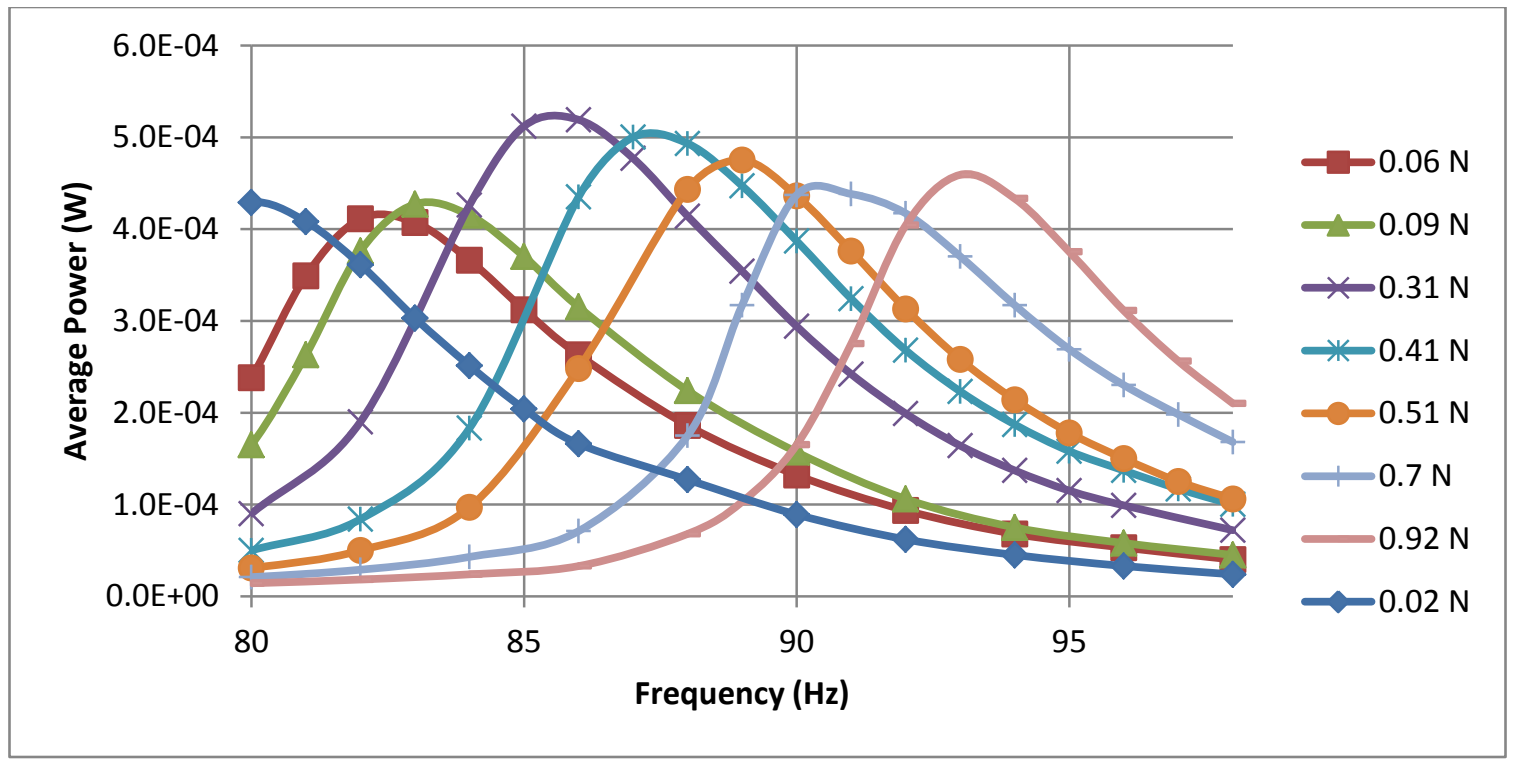

Figure 73- Relationship between average power and excitation frequencies of various tension axial load cases

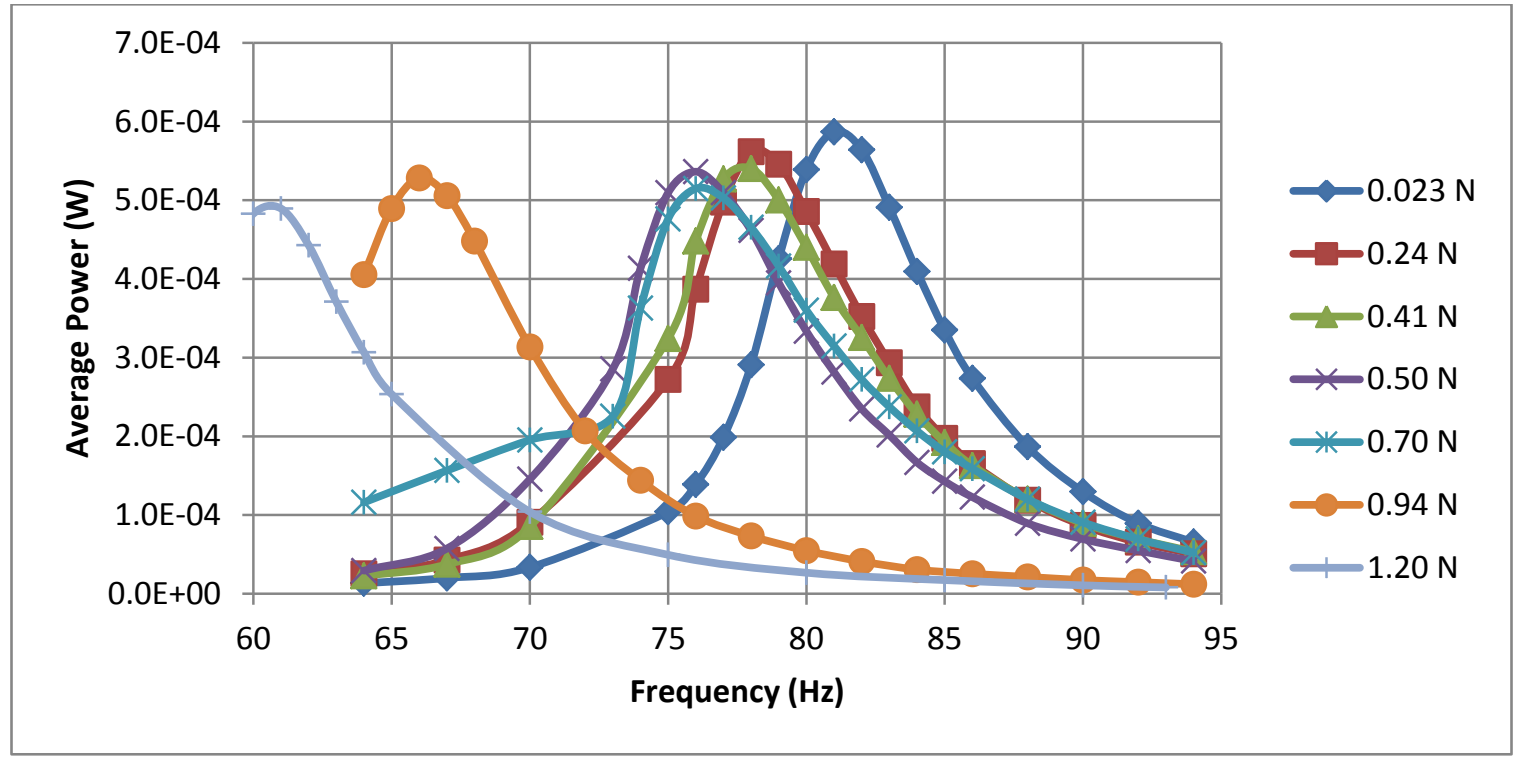

Figure 74-Relationship between average power and excitation frequencies of various compression axial load cases

\subsection{Review of Chapter 4}

Although the aim of the experiment discussed in Chapter 4 was not to verify the simulation the discrepancy between the natural frequencies and the tuning frequency range deserve some explanation. The discrepancy is caused by simplifications in the finite element model and complete reliance on material properties provided by the manufacturer. 
Another method for finding the open circuit natural frequency from the piezoelectric beam was to gently tap the beam and extract the frequency from the voltage response. The frequency was found to be $90 \mathrm{~Hz}$ in the absence of a preload. It is believed that the discrepancy could be due to the stray magnetic field of the shaker, which has a magnitude of 15 gauss.

A model considering the dimension of the tip mass instead was created and showed the expected natural frequency to be $102 \mathrm{~Hz}$. The difference between the simulation and the tap test was not significant at $102 \mathrm{~Hz}$ versus $90 \mathrm{~Hz}$. This difference may be explained by the extra mass from the glue, the moisture protection coating on the beam, the end clamp which is only an approximation of a fixed end since it still has some compliance, and/or the slight variances in the material properties provided by the manufacturer.

Another discrepancy in the results was the adjustable range of frequency. In the tensile portion, the experiment appeared to show about $11 \mathrm{~Hz}$ per Newton of force while the simulation showed about $2 \mathrm{~Hz}$ per Newton of force.

This discrepancy lies in the sensitivity of the model to the material properties. The finite element model is very sensitive to small changes in the material properties provided by the manufacturer. To illustrate the sensitivity the properties were slightly increased by 10 percent. However the range of frequency per Newton increased 4 times; from about $2 \mathrm{~Hz}$ per Newton of force to $8 \mathrm{~Hz}$ per Newton of force. In addition dimensions of the beam such as thickness of each material used the ideal dimension provided by the manufacturer. These dimensions have a tolerance range and can vary at seen in Table 2 . 


\section{Chapter 5: Simulation Studies}

Chapter 5 presents the development of a closed loop tuning system for tuning of a vibration powered harvester. A neural network is used to simulate the piezoelectric beam. The chapter concludes with case studies to show the effectiveness of the proposed system.

\subsection{Closed Loop Tuning}

The main purpose of the simulation was to investigate the feasibility of a closed loop tuning system for the adjustment of preload. Previous works relied on lookup tables for preload adjustment [47]. The benefits of a closed loop tuning system include the ability to the applied to any piezoelectric beam regardless of construction, manufacturing variances or material behaviour under different temperatures. This is more advantageous than the lookup table method as each lookup table is tailored to a specific beam. The significance of a tuning system such as this is the ability to automatically tune the beam when the excitation frequency changes by implementing some form of automated tuning mechanism which would be especially useful if the frequency changes frequently. Even if the excitation frequency changes infrequently this tuning system can give directions in aiding the manual tuning of a beam.

Initially the vibration sensor sends a signal representing the base vibrations to the controller. The voltage from the beam is also sent to the controller. The controller extracts the excitation frequency from the signal of the vibration sensor, and the beam's natural frequency from the voltage signal from the beam. It then compares the two frequencies, and sends a signal to the axial force adjusting mechanism to either increase or decrease the force applied on the piezoelectric beam. These adjustments continue until the frequencies are matched as closely as desired. A diagram of this closed loop tuning system is included in Figure 75. 


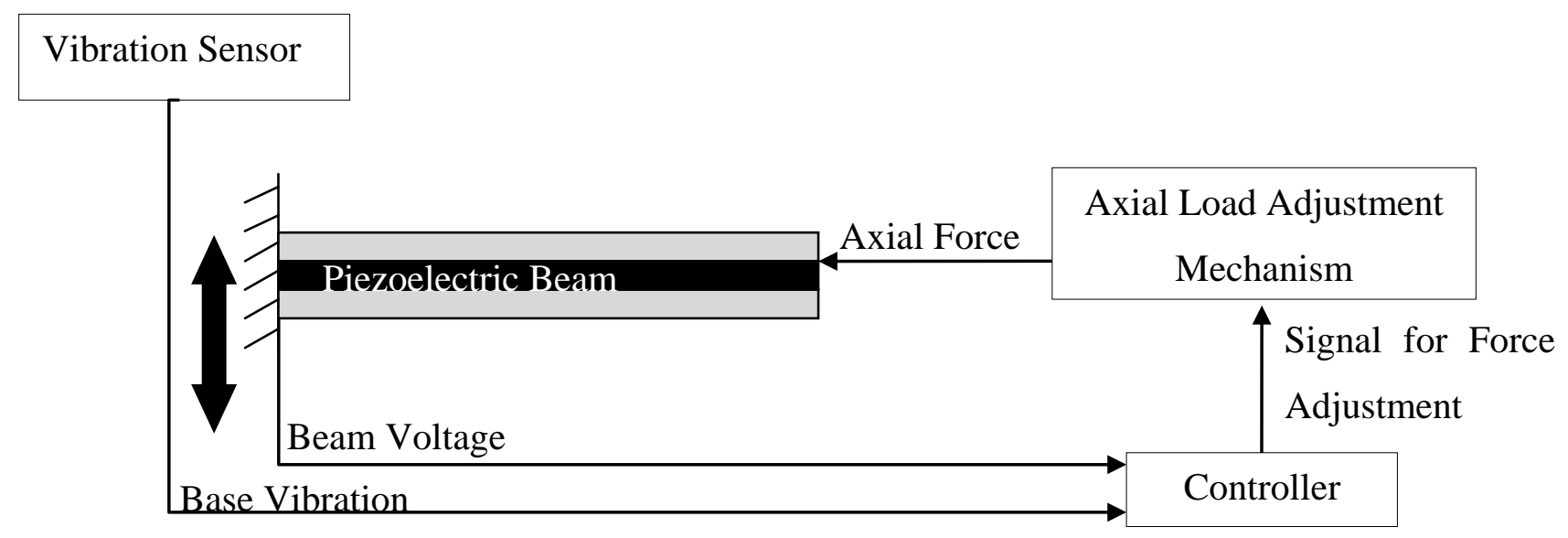

Figure 75 - Diagram of an axial load tuning system

piezoelectric beam, undergoing base excitations and having an axial load applied, can be simulated using FEA; however since a FEA program cannot run in a loop, a neural network trained on the FEA results was used instead. Shown in Figure 76 is a block diagram of the process.

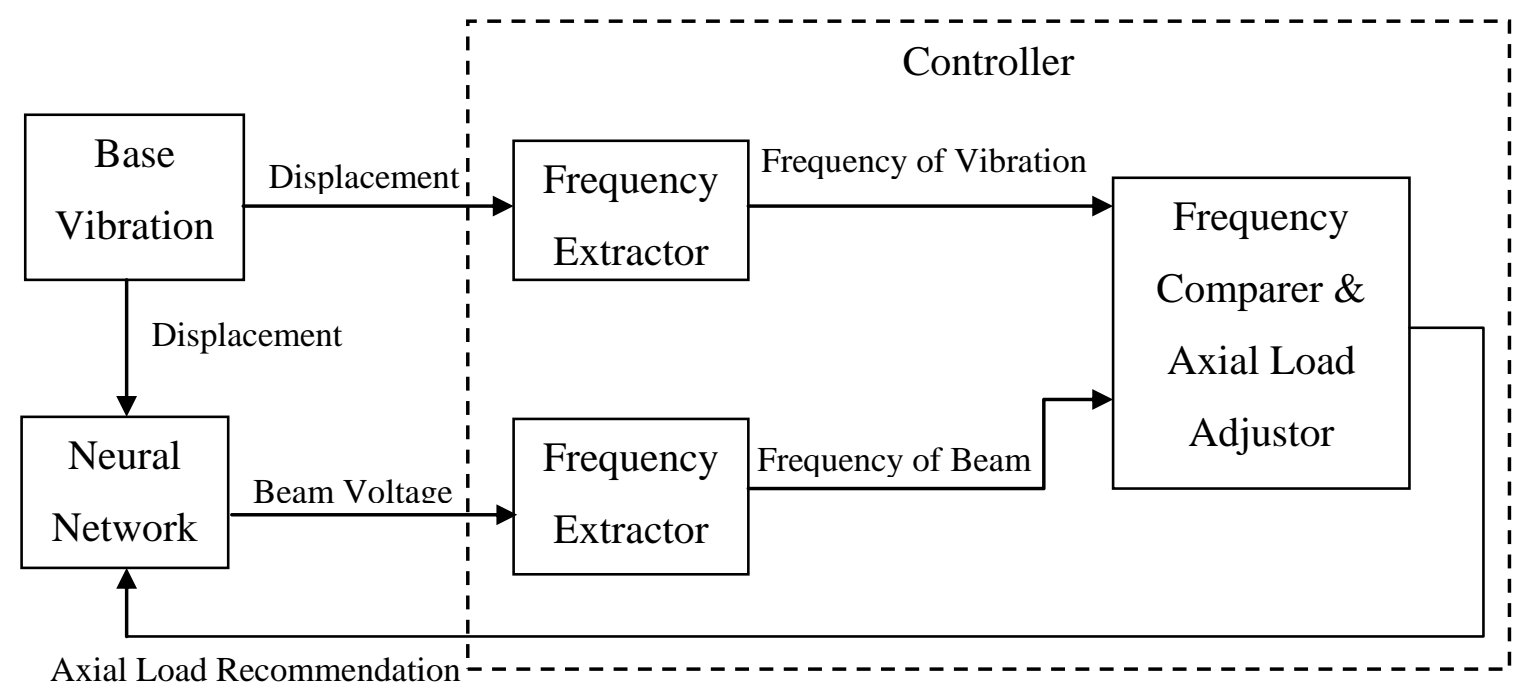

Figure 76 - Diagram of axial load tuning system simulation

The closed loop tuning system was simulated using MATLAB (Mathworks, version R2010b). The piezoelectric beam's voltage response to a base vibration and preload was simulated with ANSYS (ANSYS Inc., Academic Research, Release 14). The results from the ANSYS simulations were used to create the training sets for the neural network toolbox found in MATLAB. 
The computer model of the entire tuning system starts with an input of the base excitation vibrations, these vibrations are represented by their displacement in the time domain. The displacements are sent to an algorithm called the Yule Walker Algorithm which outputs parameters that represent the power spectral density (PSD) of the base vibrations. These parameters and the axial load make up the input into the neural network, which then outputs parameters representing the beam's voltage PSD.

The axial force inputted into the neural network is decided by the preload adjustor which is based on the vibration frequency and the beam frequency. To find the vibration frequency PSD of the displacement from the excitation vibration are found and the frequency at which the maximum peak occurs is taken to be the vibration frequency with the most power and is the target of the tuning. For the beam frequency the parameters from the neural network output are transformed back into a PSD and the peaks are read. These peaks are sent to a frequency comparer that decides whether the beam frequency is higher or lower than the vibration frequency. These results are sent to the preload adjustor to adjust the preload. This process continues to loop until the preload no longer needs adjustment when the beam and vibration frequencies match. A block diagram of the computer model of the closed loop tuning system is included in Figure 77.

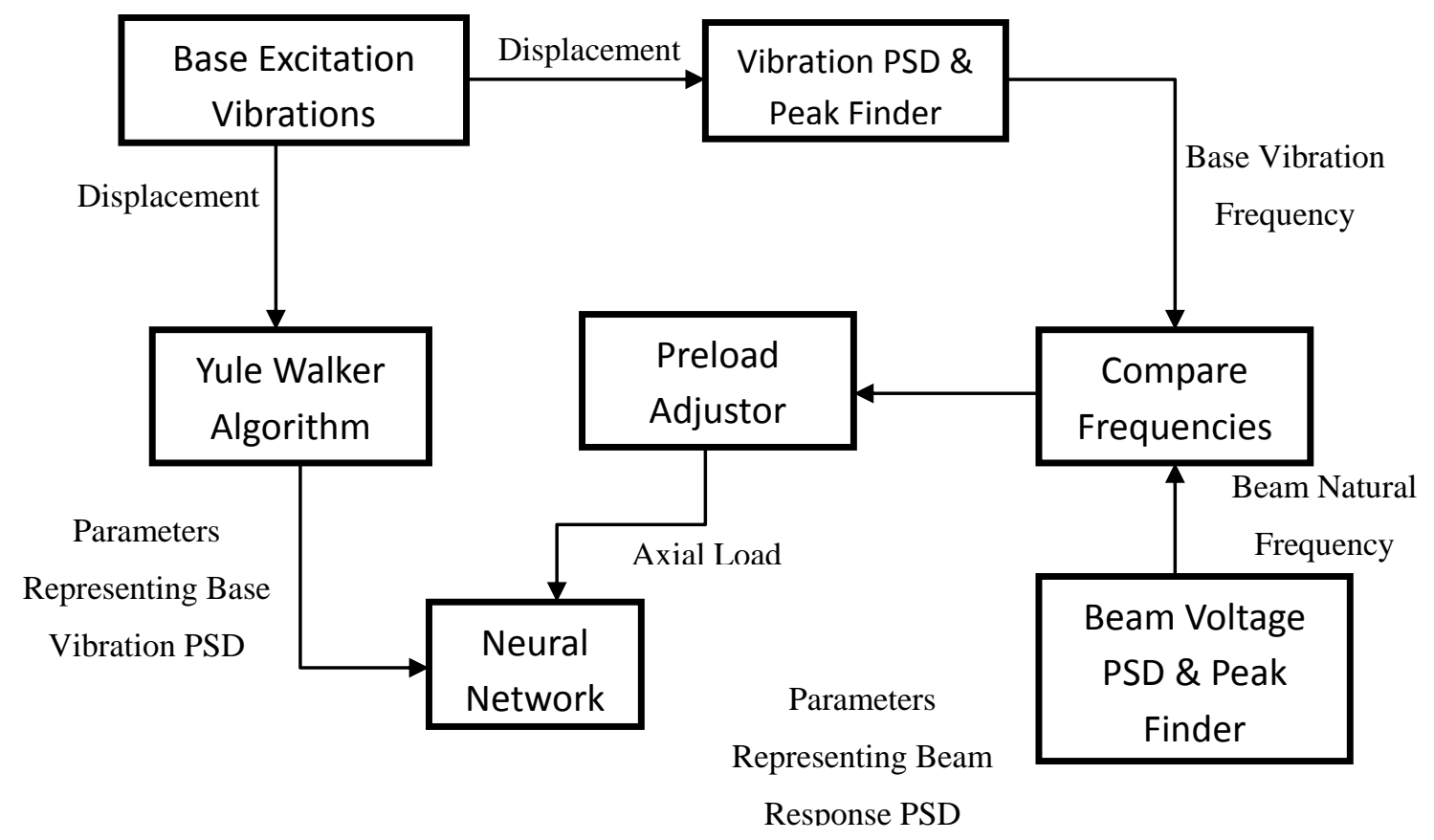

Figure 77 - Flow chart for closed loop system 
The parameters of the PSD are determined using a spectrum estimation method such as the maximum entropy [54]. The Yule Walker method was used for parameterization of both the input base displacement and voltage output spectrum [55].

An order of 10 was chosen for the Yule Walker model since the input was generated from ANSYS data. This data did not contain noise, thus relieving concerns of spurious peaks from choosing too high of an order. The order was not especially important; it had to be high enough to allow an appropriate number of spectral peaks in the PSD. Since two peaks were expected, a minimum order of four was required since the number of peaks is half the order number [56]. Order selection was based on the recommendations that $p=N / 3$ or $p=N / 2$ where $p$ is the order number and $\mathrm{N}$ is the number of data points per sample set; anything higher than that range would also work in this case [56]. The Yule Walker method was necessary to provide the inputs for the neural network. It was also possible to convert the parameters back into a PSD [57]. From the PSD the difference in power can be determined although it should be noted that this value has no physical meaning. To determine the power harvested in Watts, a resistor must be connected to the ANSYS simulation for a single run.

\subsection{ANSYS Model}

The finite element simulation is a representation of the piezoelectric beam. Unfortunately FEA programs are not able to run in a loop to continually provide the voltage output. Thus it was necessary to run a wide variety of FEA simulation to create a training set for the neural network.

The ANSYS model is the finite element model of the setup of the beam with tip mass described in 3.2.1 using the same dimensions as illustrated in Figure 55. Simplifications were made in modelling the magnet. Some features such as a protective coating on the beam could not be accurately modeled and were not included, resulting in a beam with a natural frequency of 118 Hz.

The piezoelectric ceramic (SOLID226) and structural portion (SOLID186) of the power harvesting beam were selected. Both element types are 20 node elements [58].

Material data for the piezoelectric model such as the density, permittivity, piezoelectric constants and stiffness were inputted. It should be noted that the elasticity, permittivity and piezoelectric constants are transversely isotropic and are thus entered as a matrix. The structural material, 
carbon fibre, was orthotropic; the material data inputted included density, elastic moduli, shear moduli and Poisson's ratios. The values were provided directly from the manufacturer. Afterwards, the beam was modeled as three layers placed in a stack with each layer being $38 \mathrm{~mm}$ long and $7.2 \mathrm{~mm}$ wide. The thickness of each piezoelectric and carbon layers were $0.27 \mathrm{~mm}$ and $0.24 \mathrm{~mm}$ respectively. The two piezoelectric layers sandwiching the carbon fiber layer were modelled in the software by "gluing", whereby volumes share a common area. The material data and element types were attributed to each layer accordingly and the model was meshed. For the mesh, hexahedral (brick) elements with an element size of 5.5E-4 m were chosen. The hexahedral element shape was selected because the shape of the volume to be meshed was rectangular and therefore had no curves. Thus, it was not necessary to use tetrahedral elements which require a longer solve time due to more elements per edge length. The mesh sensitivity analysis indicated that the appropriate number of elements to use was approximately 3000 . The element size of 5.5E-4 m uses 2941 elements. The mesh sensitivity analysis plot is seen in Figure 78.

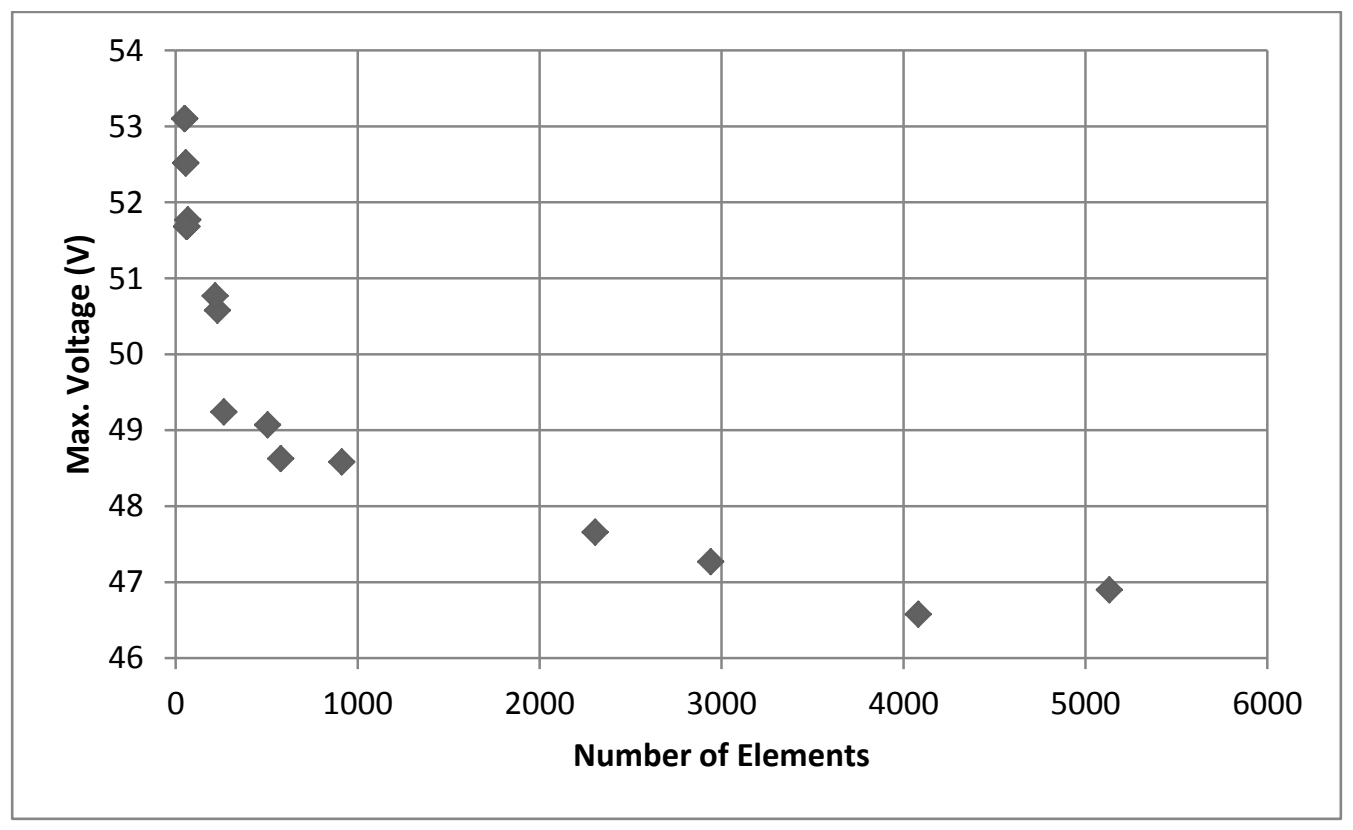

Figure 78 - Mesh sensitivity analysis to help determine the minimum amount of elements

To create the electrodes, the nodes at the top surfaces were selected then coupled form the top electrode, which meant that the voltage on the entire layer was fixed and uniform. The same procedure was followed for the ground electrode and afterwards the voltage of the ground 
electrode is set to $0 \mathrm{~V}$. The electrodes represented by a node can be connected together with a line element representing a resistor. The resistor was a CIRCU94 element. This resistor is not necessary for obtaining the voltage data for neural network training. The weight of the magnet was applied as a point element at a node at the centre of the free end of the beam, for the fixed end of the beam the $\mathrm{x}$ and $\mathrm{y}$ displacements were set to zero. However the $\mathrm{z}$ displacement to simulate the base excitation varied according to time and a sinusoidal base displacement was applied. Then the various analyses could be run on the model such as transient or harmonic analyses. A screenshot of the ANSYS software can be seen in Figure 79 below.

During the simulation, voltage output was extracted solely from a single layer of the beam. Because the piezoelectric layers were polarized in the same direction; the layers were connected in parallel. It is not possible to simulate the voltage or power output of a parallel circuit. Thus the voltage output of the two piezoelectric layers is the same as the voltage output of a single layer; in parallel connections the voltage does not add.

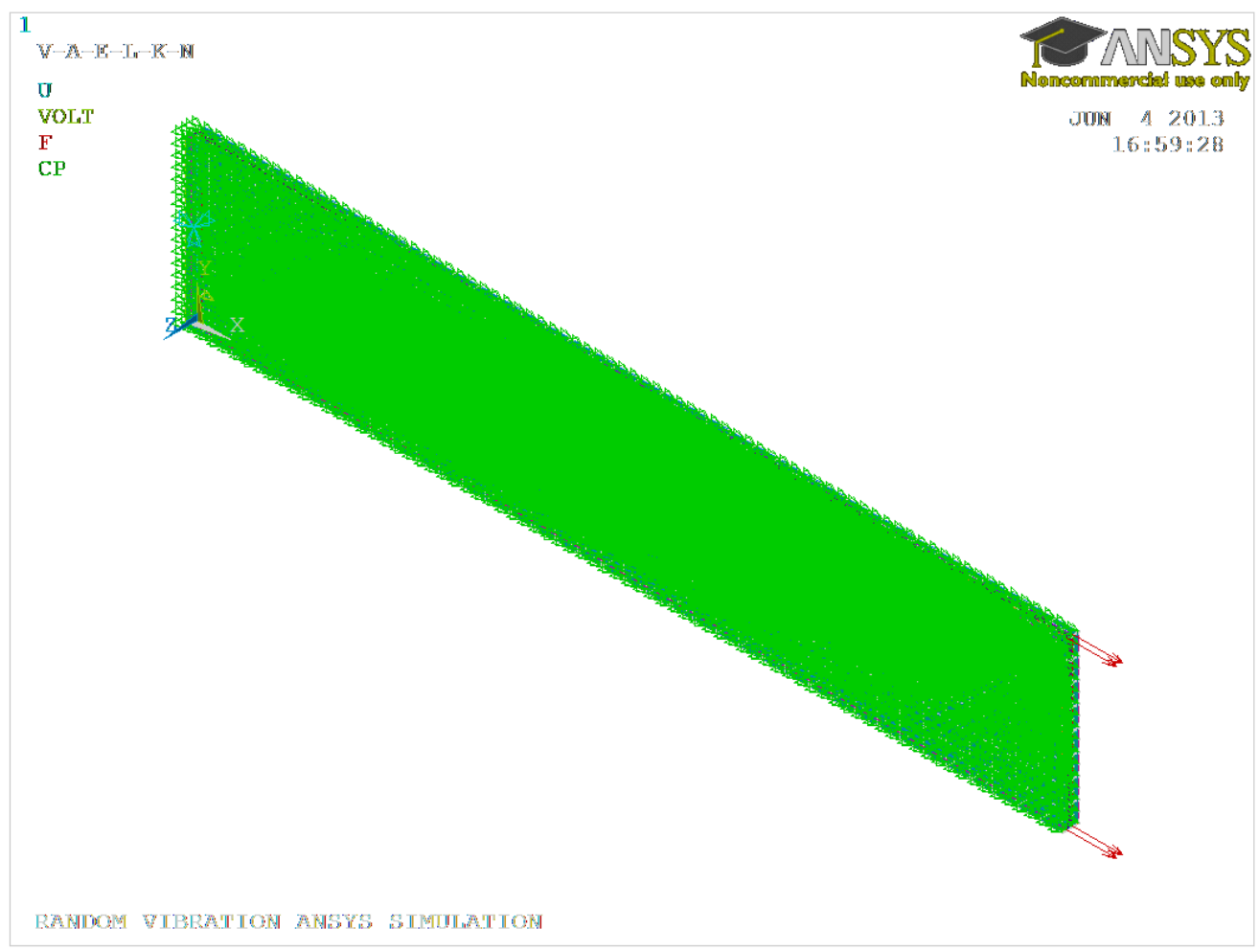

Figure 79 - Screenshot of the model in ANSYS 


\subsection{Neural Network}

The main objective of the neural network was to fit a nonlinear function between the inputs of, the vibration PSD parameters plus axial load, and outputs of the beam voltage PSD parameters. A feed forward neural network consisting of an input, hidden and output layer, was used.

The neural network toolbox in MATLAB was used for training. The input to the neural network consisted of 12 elements with 357 samples and the output consisted of 11 elements. The 12 input elements were the Yule Walker coefficients for the base excitation, the noise variance of the base excitation and the axial load. The 11 output elements were the Yule Walker coefficients for the voltage signal and the noise variance of the voltage signal. The sample data was created from a random combination of frequencies and accelerations within a range of $200 \mathrm{~Hz}$ to $13 \mathrm{~Hz}$ and 0.2 $\mathrm{m} / \mathrm{s}^{2}$ to $12 \mathrm{~m} / \mathrm{s}^{2}$ respectively. These ranges were based on Table 6 , which was from a paper published in 2003 surveying everyday ambient vibration sources [2]. Random combinations were selected using the rand function found in MATLAB which uses a pseudo random number generating algorithm to create a set of random numbers uniformly distributed from the range of 0 to 1 [57]. Random sets were chosen to create the samples, since these were adequate to train the neural network for the given range. The concern was not about a particular vibration but of the possible vibrations that could occur, thus random sets were chosen to best represent ambient vibrations in general [59]. ANSYS ran 119 random combinations and thus generated 119 samples. Since the number of samples was relatively low, the number of samples was increased by a method called regularization [60]. By introducing noise via addition and subtraction of random value of less than one percent of the data value itself, the number of samples was increased from 119 to 357. 
Table 6 - Acceleration and frequency of common vibration sources [2]

\begin{tabular}{llr}
\hline Vibration source & $A\left(\mathrm{~m} / \mathrm{s}^{2}\right)$ & $F_{\text {peak }}$ \\
\hline Car engine compartment & 12 & 200 \\
Base of 3-axis machine tool & 10 & 70 \\
Blender casing & 6.4 & 121 \\
Clothes dryer & 3.5 & 121 \\
Person nervously tapping their heel & 3 & 1 \\
Car instrument panel & 3 & 13 \\
Door frame just after door closes & 3 & 125 \\
Small microwave oven & 2.5 & 121 \\
HVAC vents in office building & $0.2-1.5$ & 60 \\
Windows next to a busy road & 0.7 & 100 \\
CD on notebook computer & 0.6 & 75 \\
Second story floor of busy office & 0.2 & 100 \\
\hline
\end{tabular}

One hidden layer was used based on the advice that "for the vast majority of practical problems, there is no reason to use more than one hidden layer[61]". In total, $80 \%$ of the samples were used for training, $10 \%$ for validation, and $10 \%$ for testing. This translated to 285 samples for training, 36 for validation and 36 for testing. To begin training, 11 hidden neurons were used based on the general rule of $\operatorname{sqrt}(\mathrm{mn})$, where $\mathrm{m}$ is the number of outputs and $\mathrm{n}$ is the number of inputs[61]. Although the results of 11 hidden neurons provided acceptable results, it would have been irresponsible if neural networks using different number of hidden neurons were not considered. After recording the training and testing errors, a graph of the error versus the number of hidden nodes, showed that at approximately 80 hidden neurons, the difference between testing and training error began to increase, indicating that 80 was the ideal number of hidden neurons. The graph of the error versus the number of hidden nodes is attached as Figure 80. 


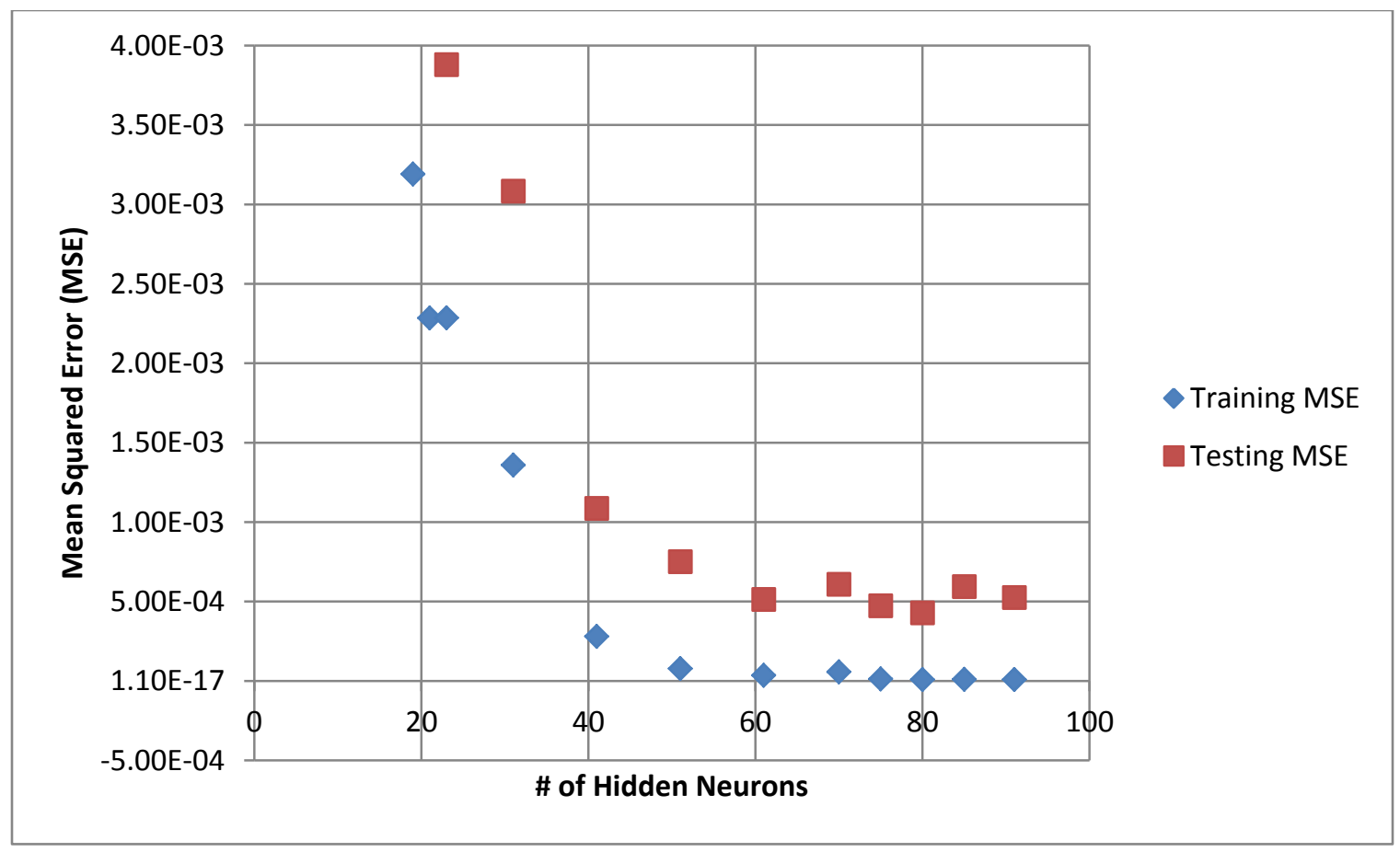

Figure 80 - Number of hidden neurons vs. error plot to help determine the ideal number of hidden neurons

Figure 81 shows the layout of the neural network showing the nodes in the input, hidden and output layer.

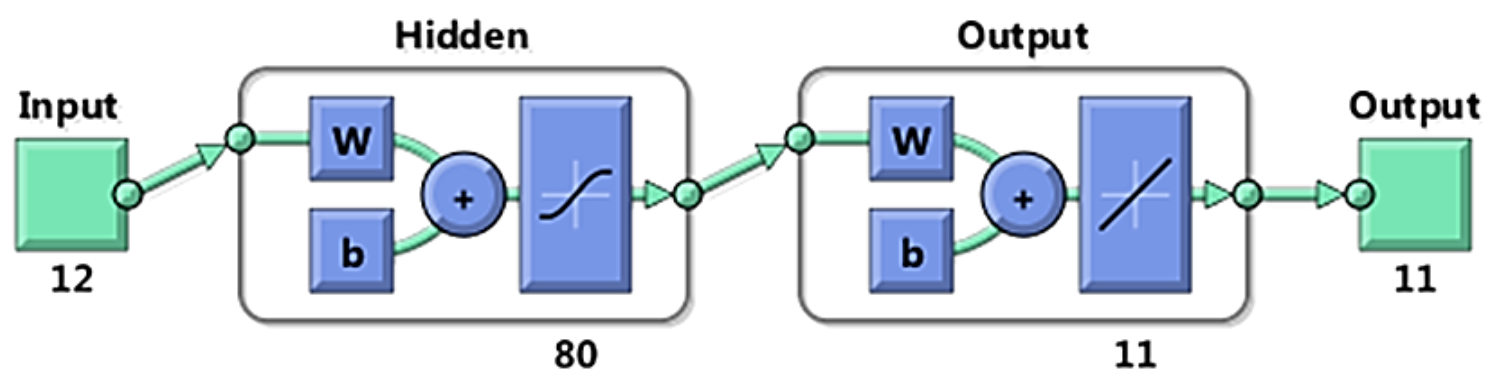

Figure 81 - Layout of neural network

The neural network tool box in MATLAB uses Levenberg-Marquardt backpropagation for training to determine the weights for the neurons [57]. This was the only algorithm that was tested since this algorithm gave favourable results. The results were favourable since the training summary, in Figure 82, indicated minimal error based on the low value for the mean squared error (MSE), an average of the squared difference between the output and target value. The regression values (R), which measured how close the output was to the target, were favourable as they were 
close to a value of 1 for all training, validation and testing. The training results for the neural network are attached below, including the error histogram, the regression plot, and the best performance plots. From the error histogram, Figure 83, it can be seen that most samples are centered on zero error, this means the neural networks has been trained accurately and no overtraining has occurred. This can also be observed in the regression plot, Figure 84, demonstrating just how closely the estimated outputs correspond to the actual outputs as witnessed in the plot, the data points all lie along the fit line. One of the obstacles in neural network training is overfitting, namely when the neural network begins to memorize the data set. This can be prevented by using a method called early stopping which is the method employed by MATLAB. Early stopping is implemented by monitoring the MSE of the validation samples. When the validation MSE increases for a few epochs, MATLAB stops training the neural network and uses the weights of the neural network at the epoch when the validation MSE is the lowest. From the training performance graph, Figure 85, it can be observed that optimal performance occurs at the $313^{\text {th }}$ epoch

$\begin{array}{lccc} & 8 \text { Samples } & \nabla \text { MSE } & \triangle \mathbf{R} \\ \text { (1) Training: } & 285 & 2.37362 \mathrm{e}-5 & 9.99960 \mathrm{e}-1 \\ \text { Validation: } & 36 & 3.72863 \mathrm{e}-4 & 9.99510 \mathrm{e}-1 \\ \text { Testing: } & 36 & 4.38324 \mathrm{e}-4 & 9.98925 \mathrm{e}-1\end{array}$

Figure 82 - Mean squared error and regression values 


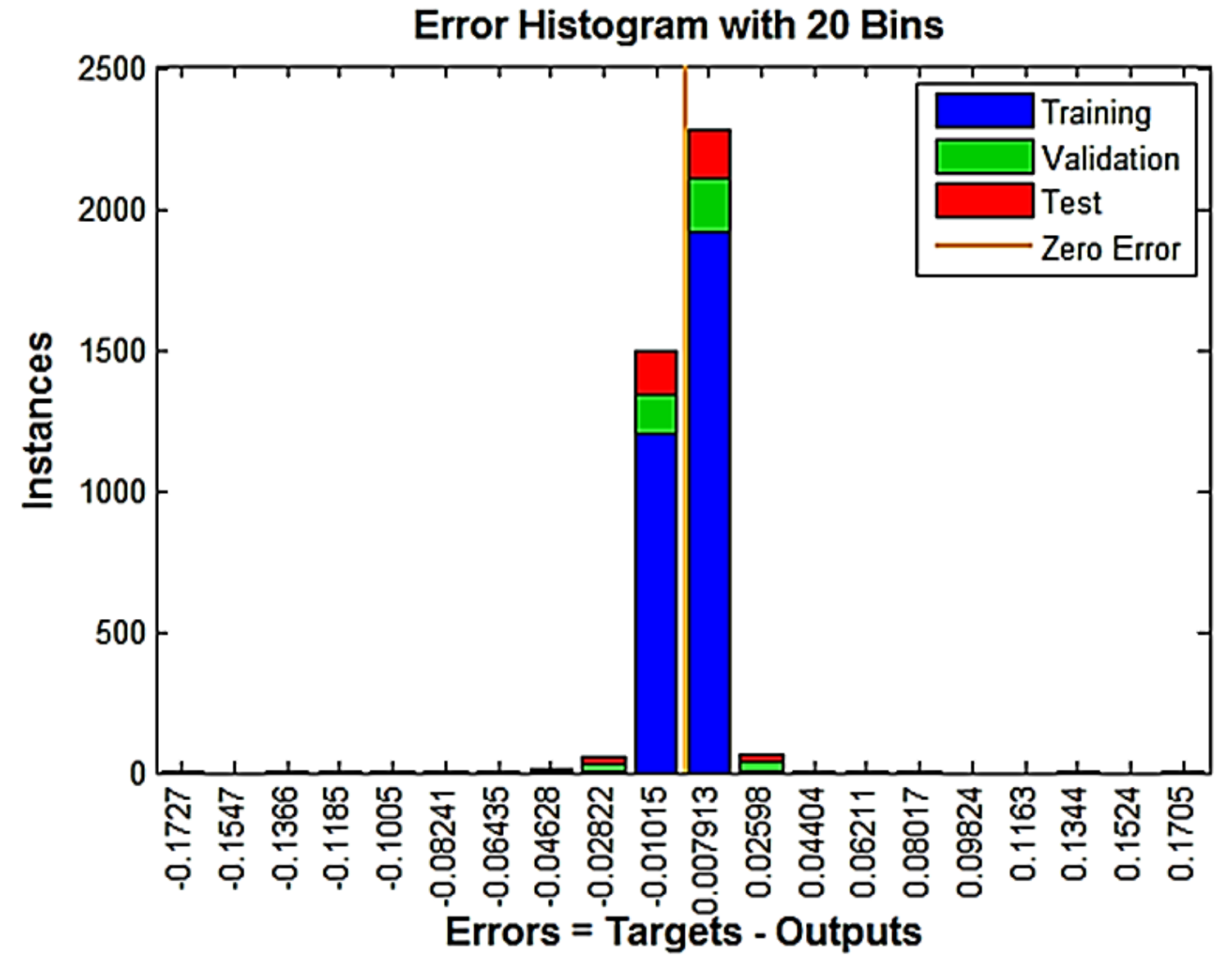

Figure 83 - Error Histogram 

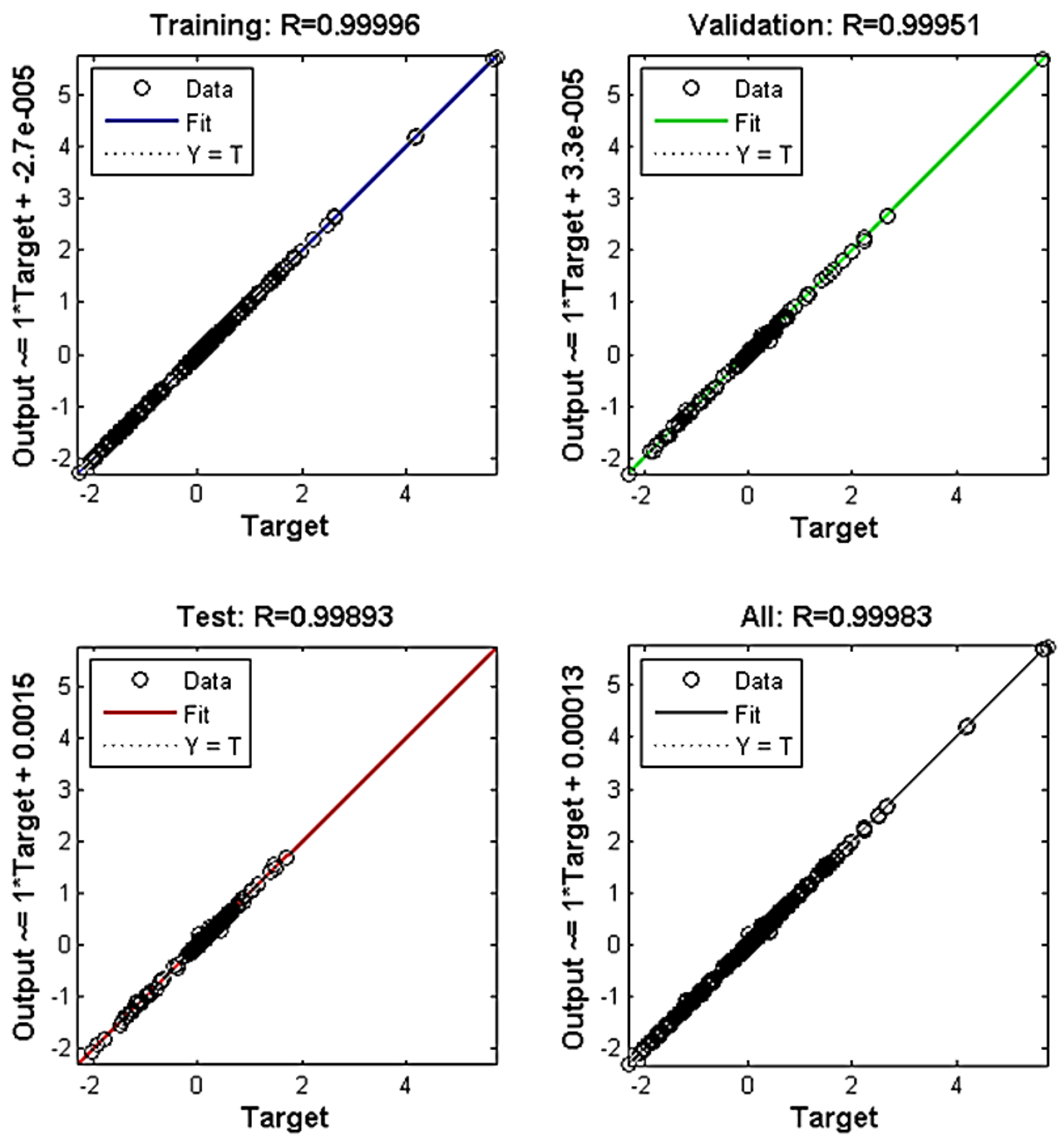

Figure 84 - Training regression plot 


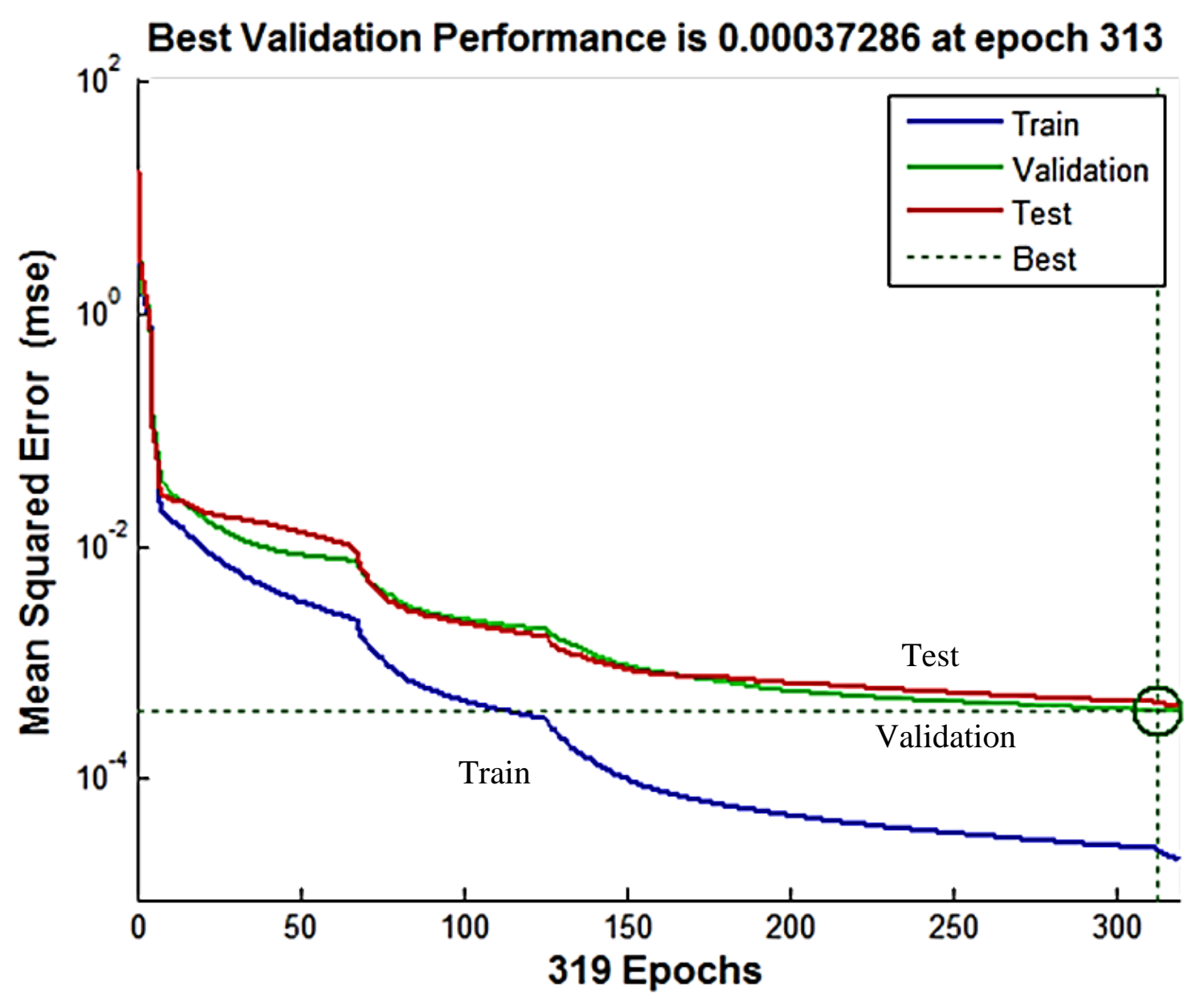

Figure 85 - MSE vs. Epochs for best performance

\subsection{Simulation of the Closed Loop System}

Simulink, a component of the MATLAB, allows the creations of block diagrams to simulate systems. This was used to create the tuning system illustrated in the flow chart of Figure 77. Another convenient feature is that it allowed the neural network to be imported into Simulink as a block. The source for the base excitation was a sine wave block; it created a signal and is sent to a buffer which collects 100 points. A sampling frequency of $1000 \mathrm{~Hz}$, thus worked out to $0.1 \mathrm{~s}$ of data. The buffered data was run through a block (FRQZ1) estimating the Yule Walker coefficients of the simulated input from the buffer. In addition the frequency of the base excitation was estimated from the coefficients and sent to the preload adjustment block (PRLD). The coefficients for the base excitation were sent through the neural network block (NNET) and the estimated coefficients for the beam voltage were generated from a trained neural network. The frequencies at which peaks occurred in the PSD were also found by the FRQZ block and 
sent to the preload adjustment block (PRLD). A threshold set at five percent of the maximum value was put on the peak detection instructions in block FRQZ so that unimportant peaks caused by noise from the neural network remained undetected. This threshold was set based on trial and error method.

The preload adjustment block is the heart of this tuning system, since this is where the tuning system decides whether to increase or decrease the axial load. The PRLD block receives two frequencies, from the block which extracts the frequency from the output of the beam (FRQZ), since the beam voltage PSD contains two peaks; one at the base excitation frequency and another at the natural frequency of the beam. In addition it receives the natural frequency from the input vibrations (FRQZ1). The since the PRLD block receives two frequencies from FRQZ it distinguishes between the natural frequency of the beam and that of the base excitation by taking the frequency with the larger difference from the excitation frequency to be the beam's natural frequency. If the beam frequency was lower than the excitation frequency, the preload was increased and vice versa. When the frequencies were, within $2.5 \%$ above or below the excitation frequency, the preload was not changed. The limitations of this model were due to accuracy of the neural network in predicting the output parameters, which is in turn affected by the breadth of training samples.

For the computer model the range of operation for the preload was programmed for adjustment between $60 \mathrm{~Hz}$ to $200 \mathrm{~Hz}$. But realistically the preload only adjusted from $-2 \mathrm{~N}$ to $4 \mathrm{~N}$ as the limit of the axial load applied, this translates to around $114 \mathrm{~Hz}$ to $125 \mathrm{~Hz}$ range in which the natural frequency can be adjusted to match the excitation frequency. Since at the edges of these limits the neural network is less reliable the tuning system sets the preload automatically adjust to $1.5 \mathrm{~N}$ in compression when the excitation frequency is below $80 \mathrm{~Hz}$ and $3.5 \mathrm{~N}$ in tension when the excitation frequency is above $180 \mathrm{~Hz}$. In addition if the tuning system attempted to increase the tension above $3.5 \mathrm{~N}$ and the compression above $1.5 \mathrm{~N}$ it was prevented from doing so, the reason for this is for to prevent the beam from becoming overload which can lead to failure.

One of the simulation's shortcomings was the fact that the model was only as accurate as the training data for the neural network. Also the generation of the training data for the computer model was quite time consuming, at approximately 2.5 hours per sample. Due to time constraints, the number of training samples for the neural network was limited. The utilization of a greater 
number of training samples will increase the robustness of the neural network. A screenshot of the Simulink program is included as Figure 86. 


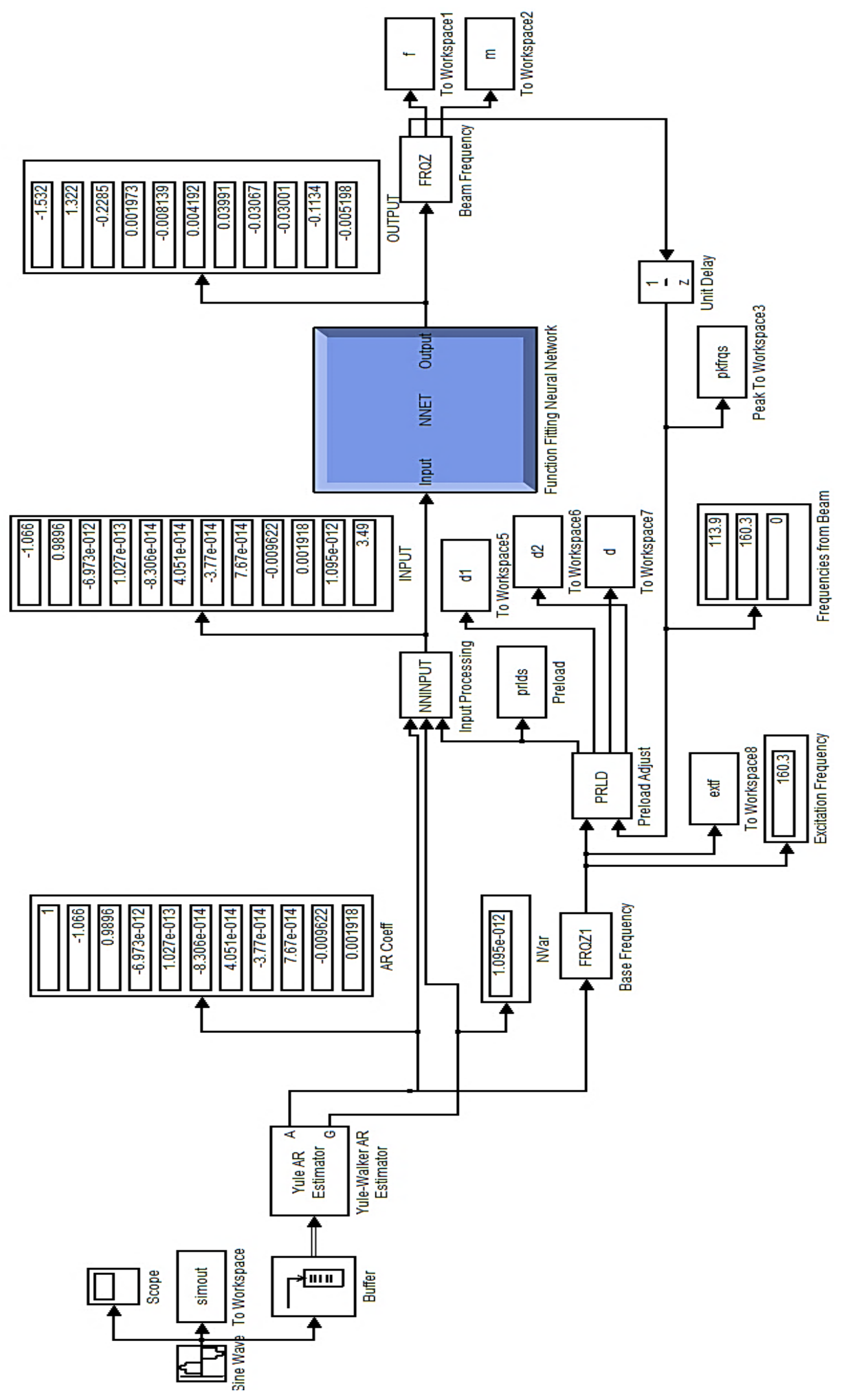

Figure 86 - Screenshot from Simulink model of the closed loop system 


\subsection{Results}

The power spectral density value outputted by the Simulink model has no real physical meaning, thus only way to estimate power output was to run a finite element simulation with an extra element to represent a resistor. Several test cases were run with an untuned scenario and a tuned scenario from the recommendations of the tuning system. Both tuned and untuned modes were tested in ANSYS in order to compare the power value being harvested.

As many previous researchers have found, there is an optimal resistance value which produces the maximum power output. Using ANSYS to run multiple harmonic sweeps, the optimal resistance value for the current device, without an axial load was $17.5 \mathrm{k} \Omega$. Figure 87 is a plot of the power versus the resistance value which indicates that $17.5 \mathrm{k} \Omega$ is the optimal resistance value.

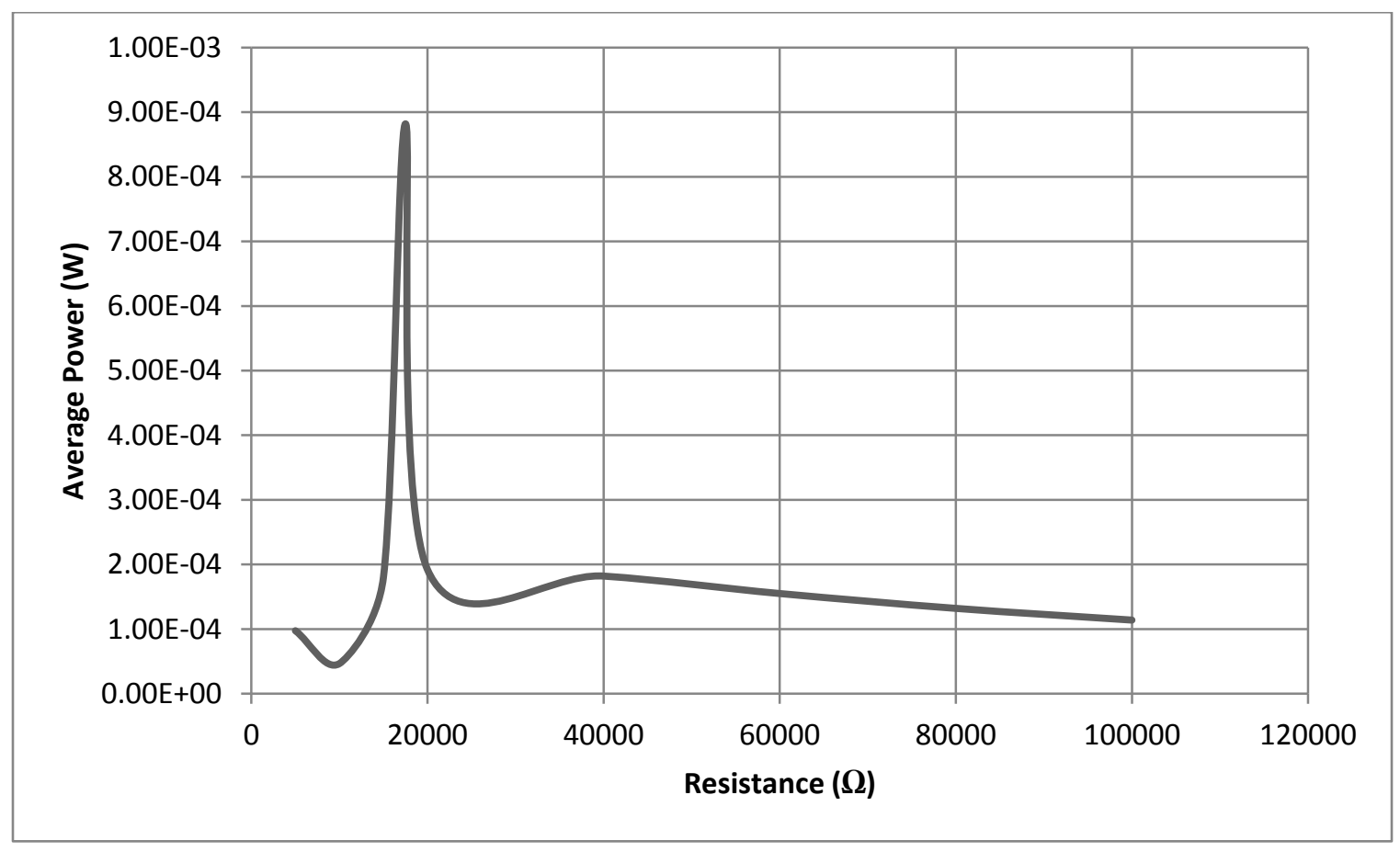

Figure 87 - Power vs. Resistance to find the optimal resistor value

As the main goal of the computer model is to simulate preload tuning, various cases were tested to demonstrate the preload picking effectiveness. 
Between the cases, the acceleration, frequencies and initial axial load starting point were varied, however electrical resistance was kept constant since it is not possible to adjust resistance in reality. The optimal resistance value of $17.5 \mathrm{k} \Omega$ was chosen for all test cases.

For the first test case, the frequency was set at $124 \mathrm{~Hz}$, the acceleration was $1.5 \mathrm{~m} / \mathrm{s}^{2}$, and the initial starting load was $1 \mathrm{~N}$. The model predicted the optimal preload to be $2.94 \mathrm{~N}$. Figure 88 shows the tuning steps of the tuning program for case 1 .

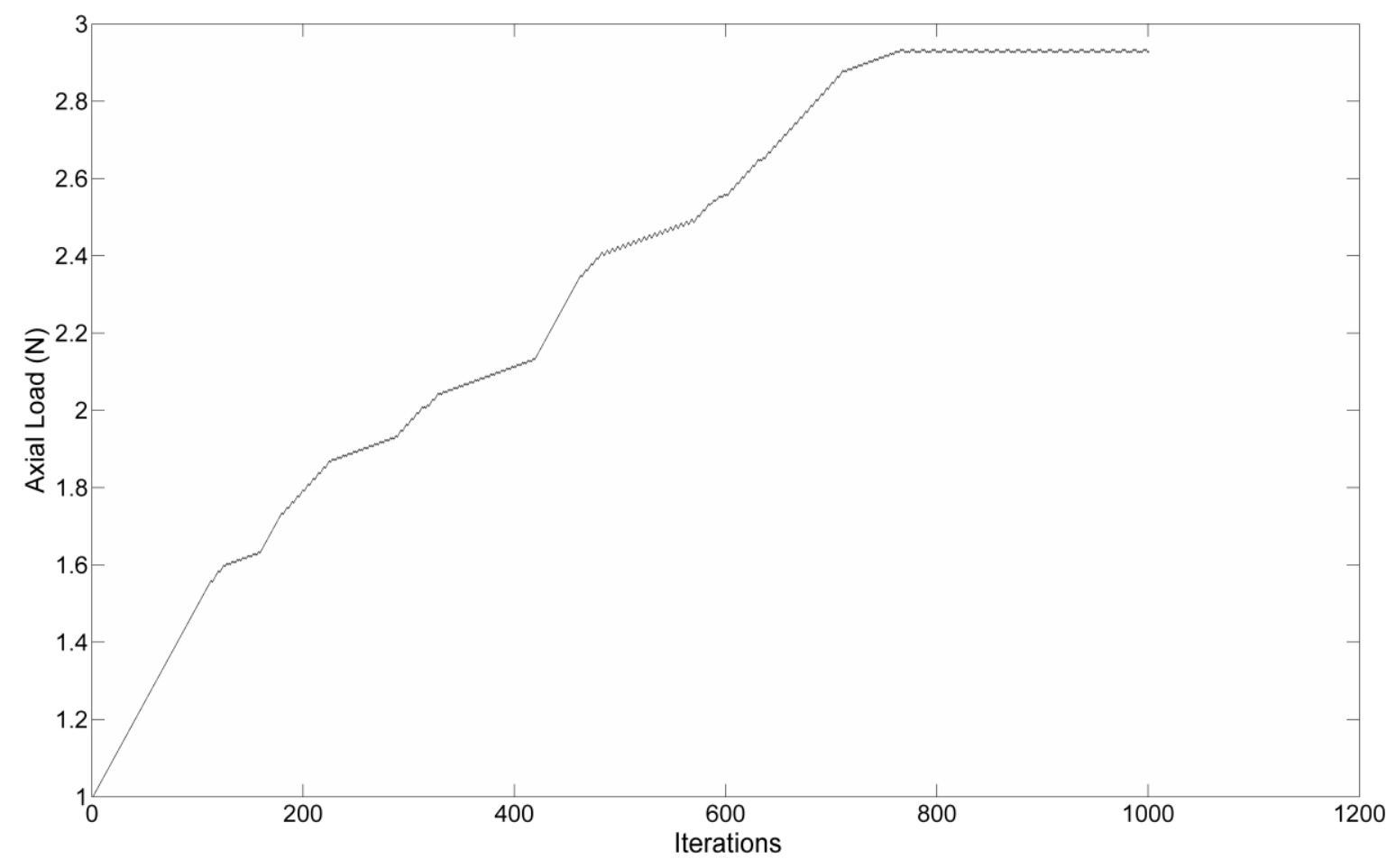

Figure 88- Axial load iterations from Simulink for case 1 
For the second test case the frequency was $122 \mathrm{~Hz}$, acceleration was $3.9 \mathrm{~m} / \mathrm{s}^{2}$, and the initial starting load was $2 \mathrm{~N}$. The computer model tuned the harvester to $2.34 \mathrm{~N}$. Figure 89 shows the tuning steps of the tuning program for case 2 .

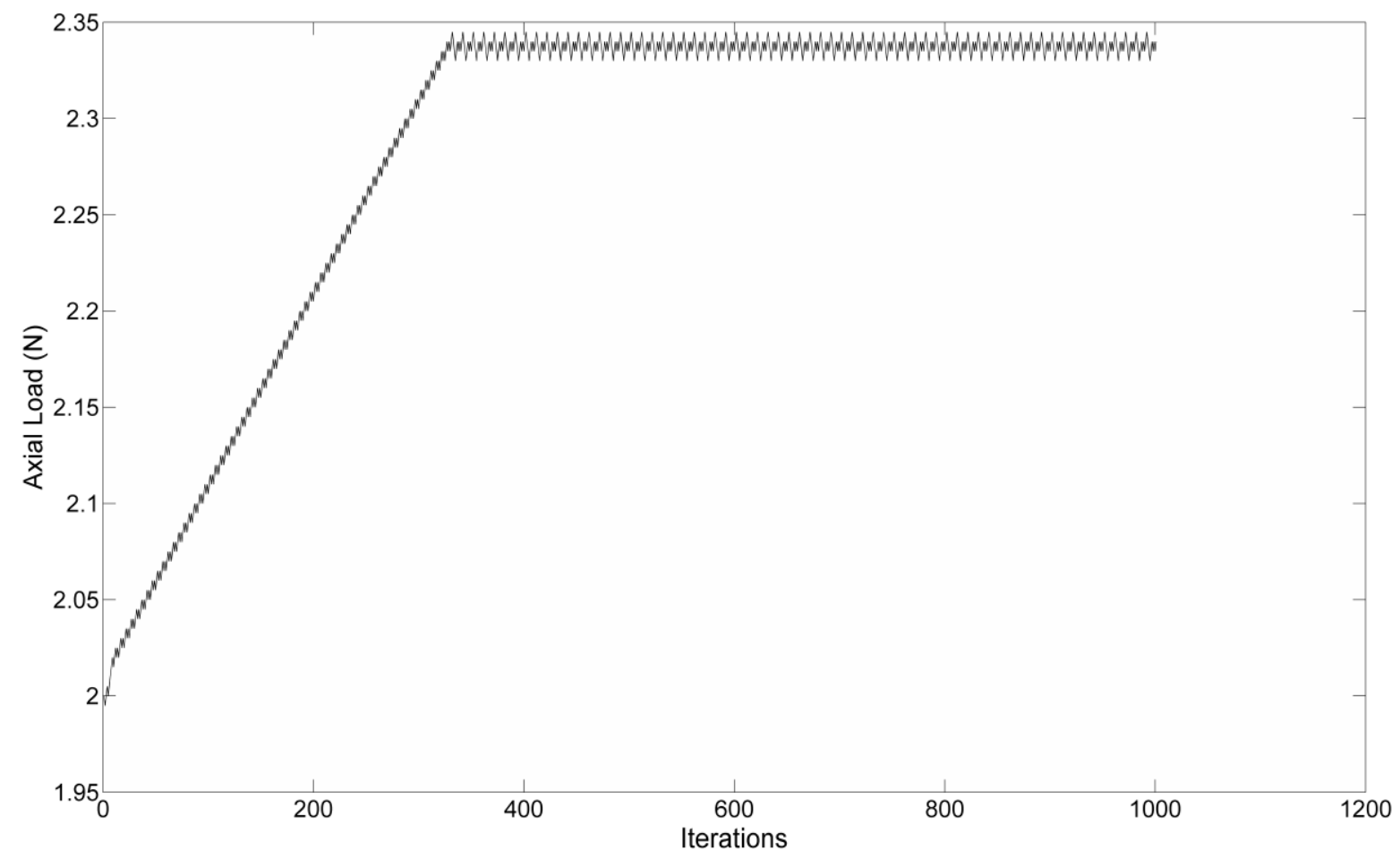

Figure 89 - Axial load iterations from Simulink for case 2 
For the third test case the frequency was $90 \mathrm{~Hz}$, acceleration was $7 \mathrm{~m} / \mathrm{s}^{2}$, and the initial starting load was $1.1 \mathrm{~N}$. For this case the computer model tuned the load to $-1.5 \mathrm{~N}$. Figure 90 shows the tuning steps of the tuning program for case 3 .

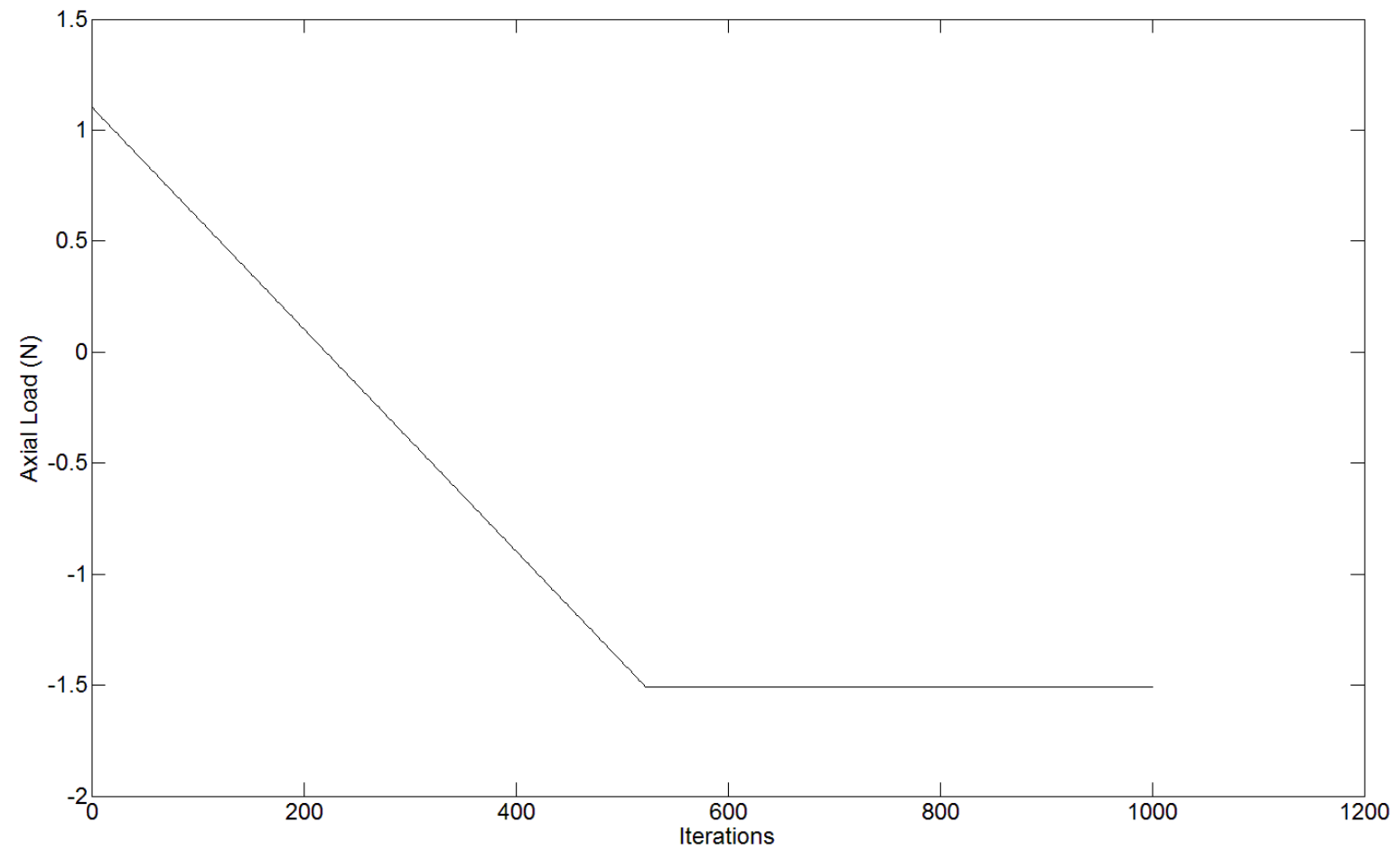

Figure 90 - Axial load iterations from Simulink for case 3 
For the fourth and final test case, the frequency was $160 \mathrm{~Hz}$, the acceleration $9 \mathrm{~m} / \mathrm{s}^{2}$, and the initial starting load $0.75 \mathrm{~N}$. This final tuned axial load was set to be $3.5 \mathrm{~N}$. Figure 91 shows the tuning steps of the tuning program for case 4.

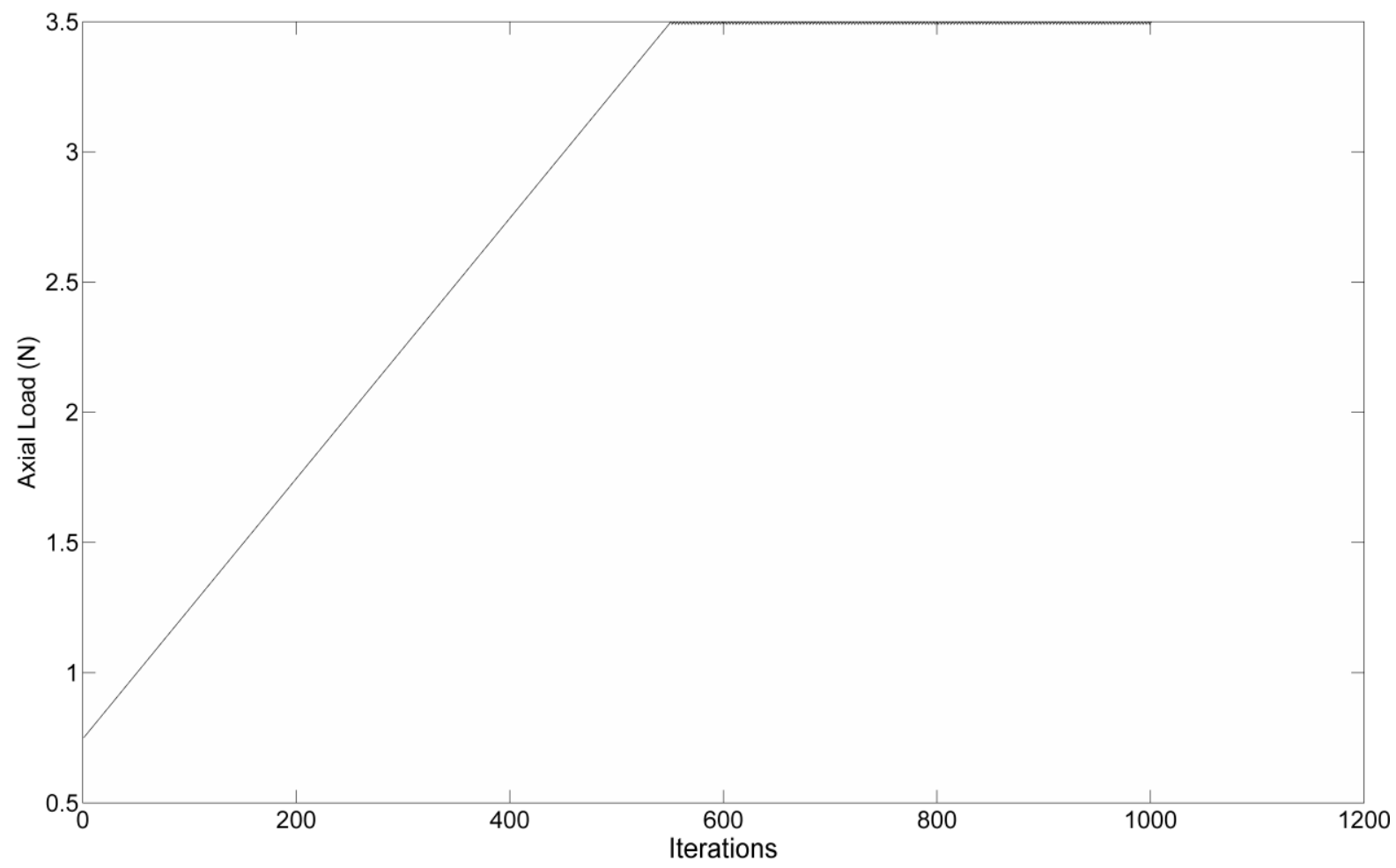

Figure 91- Axial load iterations from Simulink for case 4

ANSYS simulations were run for each test case's tuned and untuned scenarios. From the ANSYS simulations, the voltage versus time plot was found and the average power was calculated for both scenarios for each case. The formula for calculation average power is shown below. Where $\mathrm{P}$ represents power in Watts and $\mathrm{V}_{\mathrm{rms}}$ is the root mean squared of the voltage and $\mathrm{R}$ represents the resistance value in Ohms which in this case is $17.5 \mathrm{k} \Omega$.

$$
P=\frac{V_{r m s}^{2}}{R}
$$

Shown below is a column plot showing the comparison between the power of the tuned and untuned scenarios. As shown in the plot below, Figure 92, the tuned scenario consistently produces more power in all test cases, demonstrating that the axial load picking of the tuning system is effective. The results of each case in recorded in Table 7. 


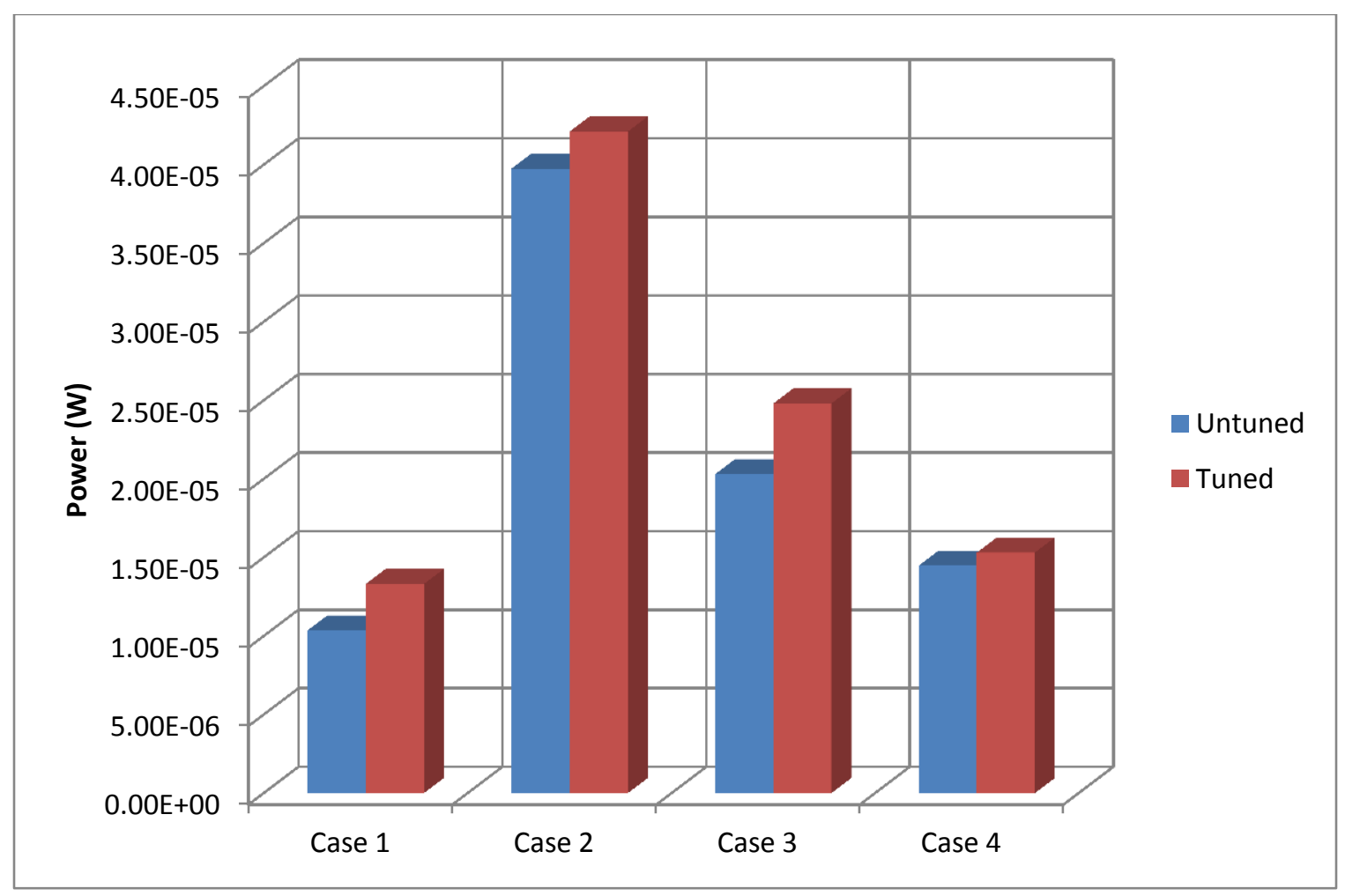

Figure 92 - Comparison of the power harvested from untuned and tuned scenarios of each case

Table 7 - Power values of the untuned and tuned scenarios

\begin{tabular}{lcccc}
\hline & Case 1 & Case 2 & Case 3 & Case 4 \\
\hline Untuned Power (N) & $1.04 \mathrm{E}-05$ & $3.97 \mathrm{E}-05$ & $2.03 \mathrm{E}-05$ & $1.45 \mathrm{E}-05$ \\
Tuned Power $(\mathbf{N})$ & $1.33 \mathrm{E}-05$ & $4.21 \mathrm{E}-05$ & $2.48 \mathrm{E}-05$ & $1.53 \mathrm{E}-05$ \\
Improved \% & $22 \%$ & $6 \%$ & $18 \%$ & $5 \%$ \\
\hline
\end{tabular}

The table above shows the amount of power generated. Although the values that appear relatively low, the results are comparable to power levels generated by the piezoelectric harvesters of the same scale reported in previous research works. In addition some cases show a greater percentage of power increase, which can be due to several factors including the initial axial load starting value being located close to the final preload value as in case 2 or because the loading frequency is out of range of the harvesters as in cases 1 and 4 and thus the percentage of improvement may seem not as impressive. 
The results have shown that the tuned scenario generated greater power than the untuned scenario for all cases examined. This demonstrates that the closed loop tuning model is able to properly adjust the axial load. It is safe to conclude that the model has met its objective of simulating a tuning system for axial load adjustment for the tuning of a piezoelectric harvester.

\subsection{Review of Chapter 5}

A potential application for the tuning system is to tune power harvesters in a car using engine vibrations. As the engine rpm changes the vibrations would change too. An automated tuning system would be desirable. Even if a tuning mechanism were not designed for automated tuning, the closed loop tuning system can aid in manual tuning. In an environment where the excitation frequency does not change often, manual tuning can be used. The closed loop tuning system in this case would be able to recommend to the operator whether to increase or decrease the axial force being applied. In this case the operator takes the place of the automated tuning mechanism.

A concern for an automated tuning system is the amount of energy used for the operation as the power harvested may not be enough to cover the power expensed in the operation of the tuning system. Previous researchers also faced this issue. It was recommended that a recovery time is required after tuning to allow for the system to recover the energy expensed; continuous tuning cannot be applied [33]. The recovery time would be based on the maximum energy cost, which is the maximum magnet adjustment and the cost of the controller and sensor. This energy cost divided by the tuned power would give the minimum time required to recover the energy and break even, provided that the vibration frequency does not change. In an environment where the vibration frequency changes constantly this system may not perform well as a recovery time is essential. Also providing a sleep mode, where energy drained by the controller and sensor are reduced, during this recovery time could save power [62]. Another common sense method is to specify controllers, sensors and design tuning mechanisms which are frugal with energy expenditure.

Although the frequency values from the experiment and the simulation do not match, the experimental results provide a more optimistic view of tuning with the axial load method. Simulations do not present as positive of an outlook, The Simulink simulation model use a simplified FEA model for training the neural network with the mass applied on a single node at tip of the beam, which doesn't fully reflect the dimension of the prototype. The major reason as 
to why this model was used for training was due to the fact that when the magnet is modelled onto the beam it prevents the piezoelectric element from solving for the voltage.

However there are various adjustments that are required for a more accurate model for the FEA model used for training. Adjustments such as finding the material properties and measuring the actual thickness of the layers. These are all outside the scope of this project. The FEA model served its purpose in creating a neural network for the testing of a closed loop tuning system. 


\section{Chapter 6: Conclusion}

A two part research work to investigate the tuning of piezoelectric power harvesting was performed; a closed loop tuning system was designed with a combination of ANSYS results, neural networks and a Simulink model. It was shown that a closed loop tuning system was capable of predicting the appropriate axial load of the beam, which tuned the beam for increased power output.

The close loop tuning system was shown to be viable by running test cases in a computer simulation of the closed loop tuning system. The result of these test cases showed that when the axial load recommended by the tuning system is simulated in FEA the output power was greater than the power generated when a random axial load was applied. Four random test cases were run and the closed loop tuning system was able to provide greater power in all test cases. This method is an improvement of the previous tuning method of utilizing lookup tables as this closed loop tuning system can be applied to any cantilevered piezoelectric power harvester.

The second part of the thesis is the results from a prototype which applied the axial load via permanent magnets. Results from the prototype showed a frequency range of $67 \mathrm{~Hz}-93 \mathrm{~Hz}$ for an axial load range of $-1.2 \mathrm{~N}$ to $0.94 \mathrm{~N}$, is achieved. Furthermore, tuning the beam from a nearly absent load to the maximum can at its most extreme condition increase the power harvested by more than seven fold.

The experiment was run to measure the power increased in axial load tuning. It builds upon the previous research, by quantifying the amount of extra power, which can be harvested through axial load tuning. The quantification was possible by the physical prototype created which applied the axial load by adjusting the distance between a permanent magnet placed on the beam and another magnet placed opposite on an adjustment screw.

An observation during the course of the experiment is that the axial load applied to beams did not seem to affect the optimal resistance value of the beam. Thus the resistance which gives the maximum power output is dependent only on the beam and the axial load applied to the beam has no effect on the resistance value for maximum power.

The contributions of this project are therefore, as follows: 
- Demonstrated the feasibility of a closed loop tuning using axial load

- Quantified the power increase in response to tuning.

- Found that the optimal resistor value does not change significantly when the axial load varies.

The future work of this project should focus on:

Applying the principle of axial loading to MEMS scale piezoelectric beams would largely benefit the field of MEMS power harvesting research.

Implementation of the closed loop tuning system with a physical prototype such that the distance of the magnets can automatically adjusts. At this point, the closed loop tuning exists only in simulation thus implementation in a prototype would serve as a logical next step.

The final crucial step is deciding how to store the energy harvested by the piezoelectric beam. 
APPENDIX A: Mechanical Drawings 


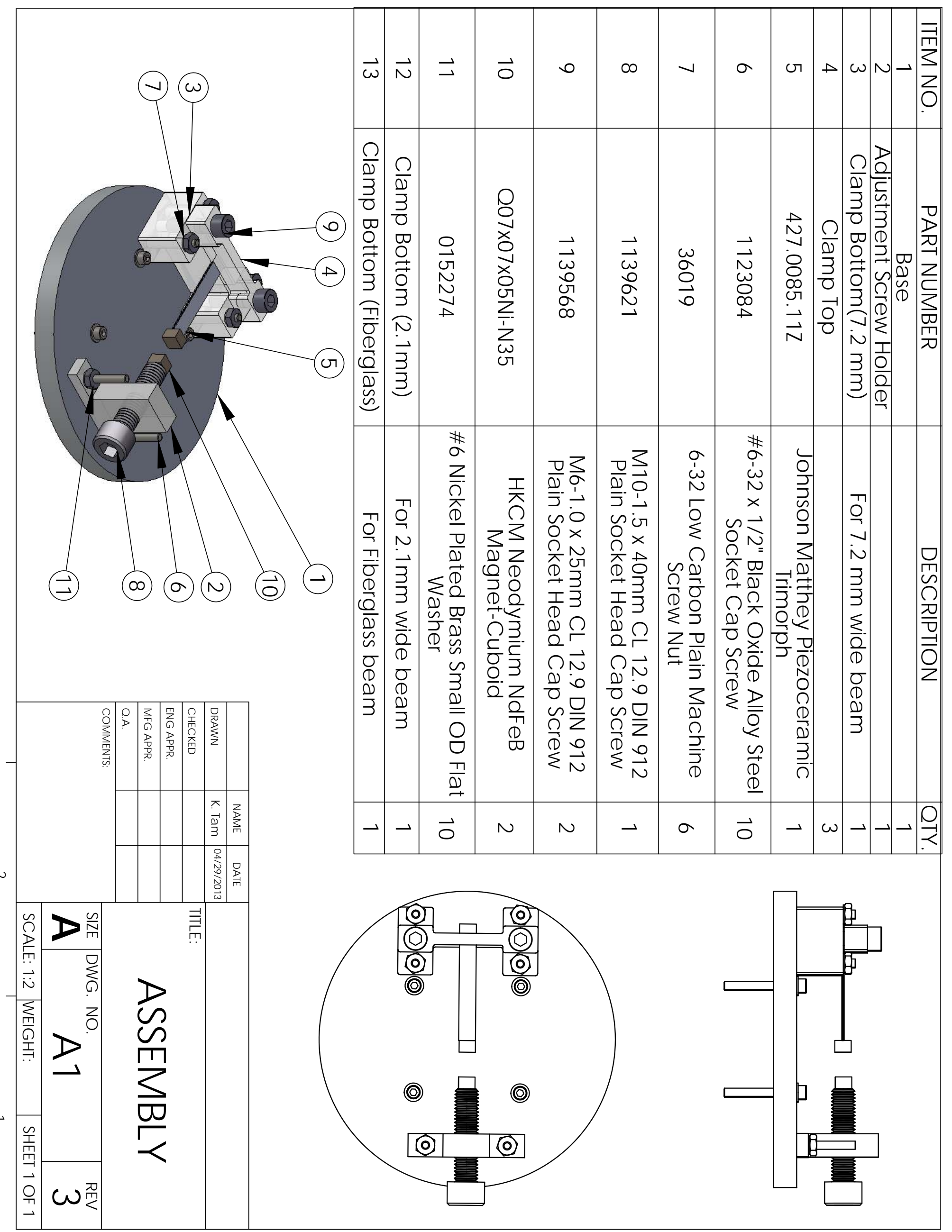




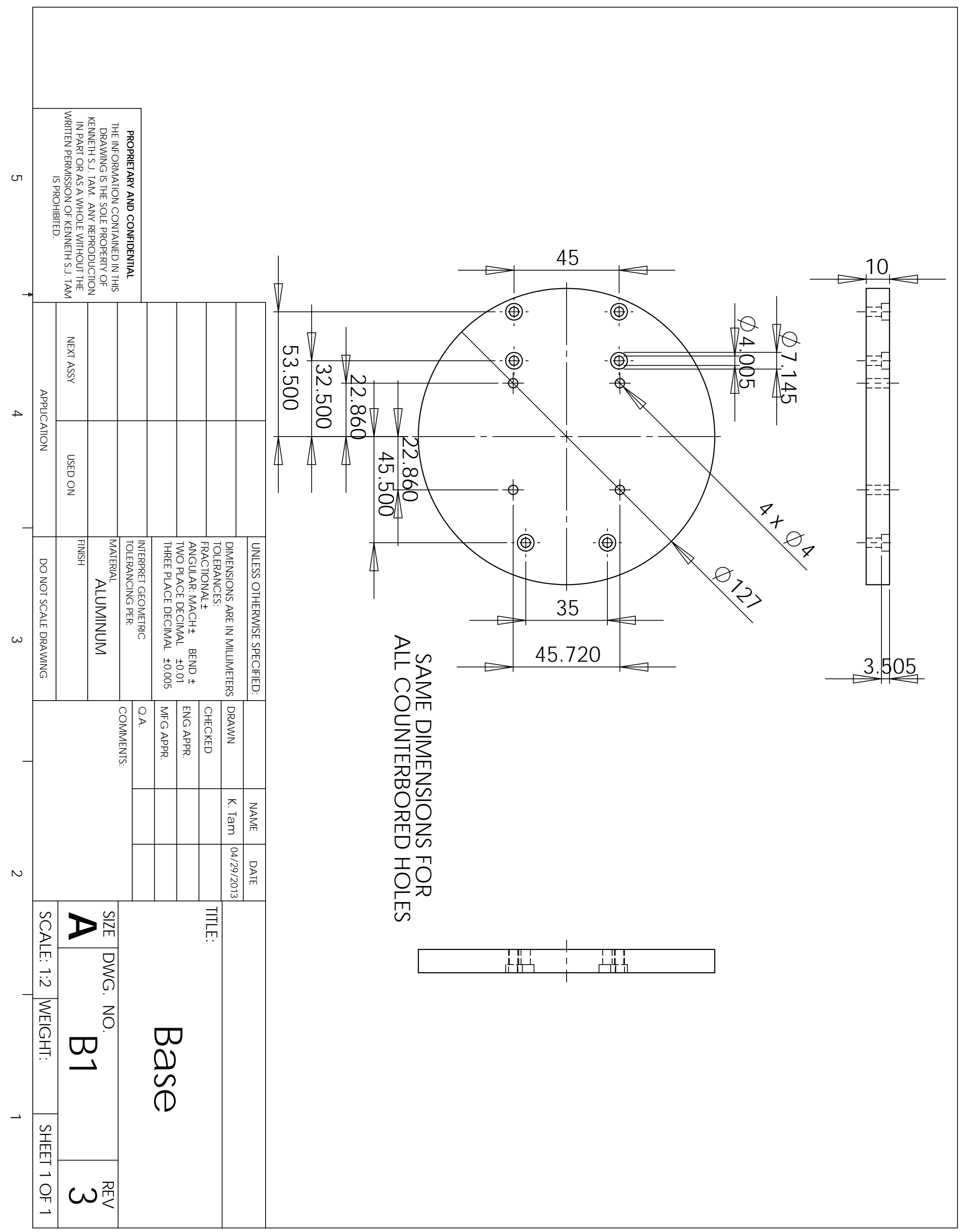




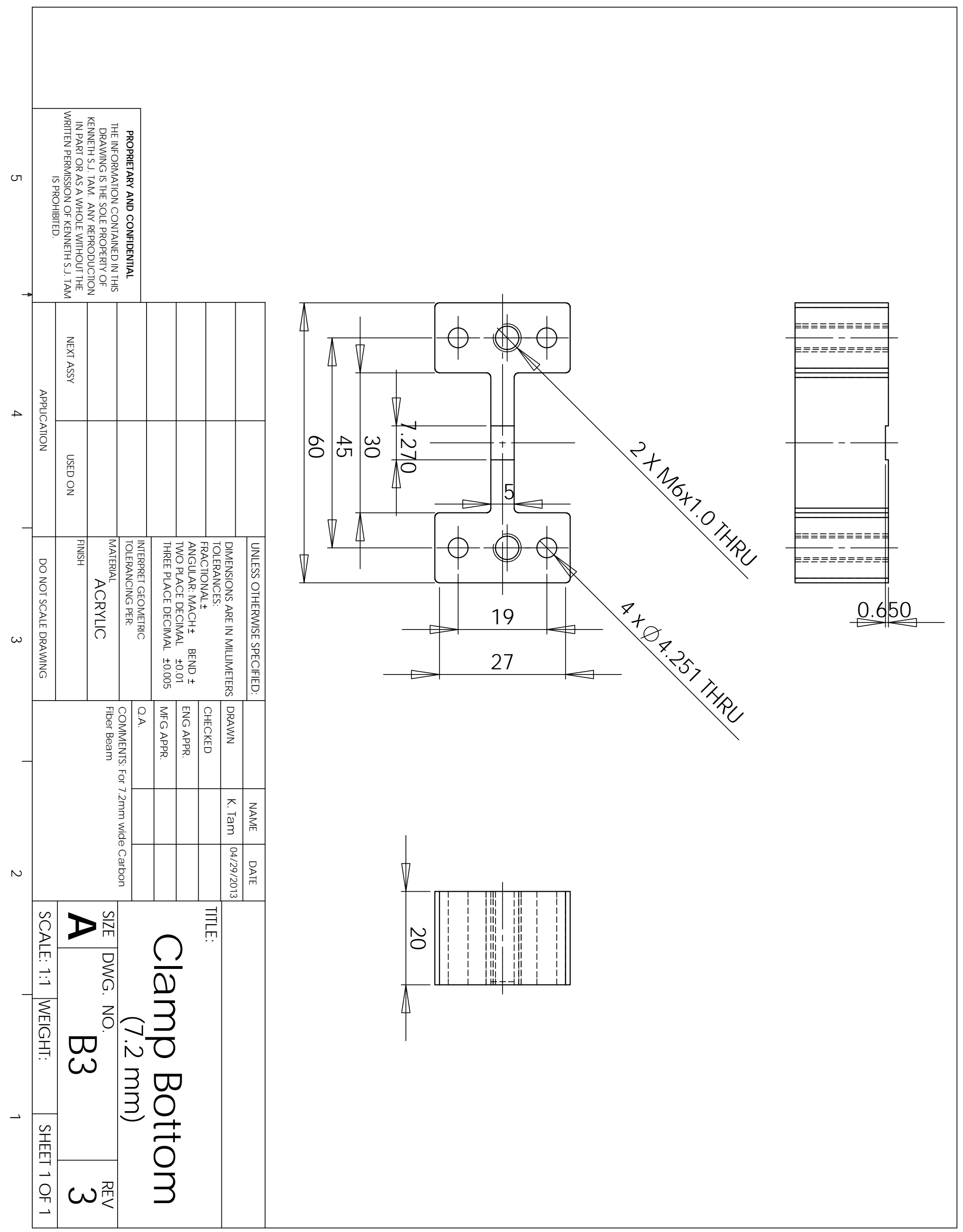



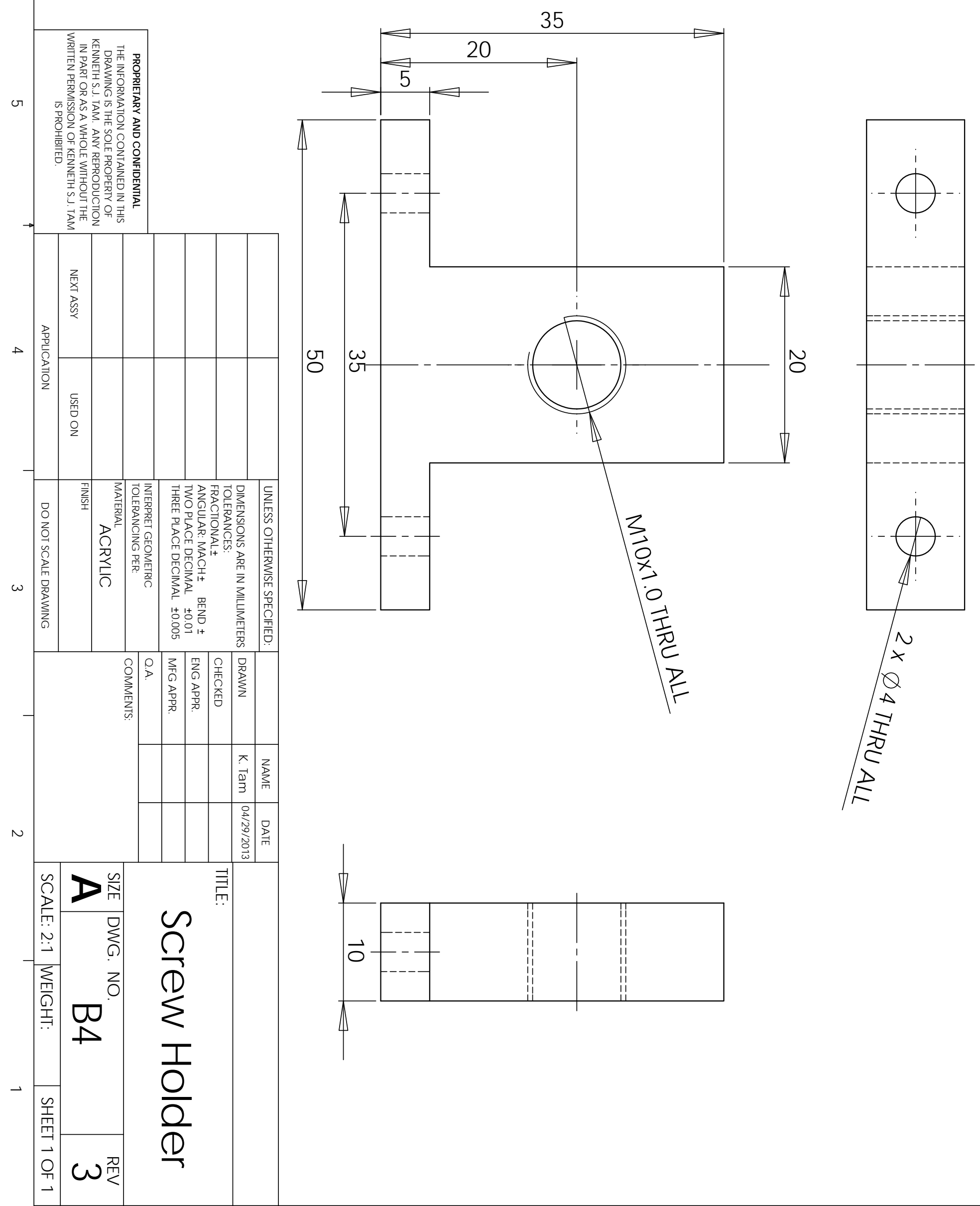


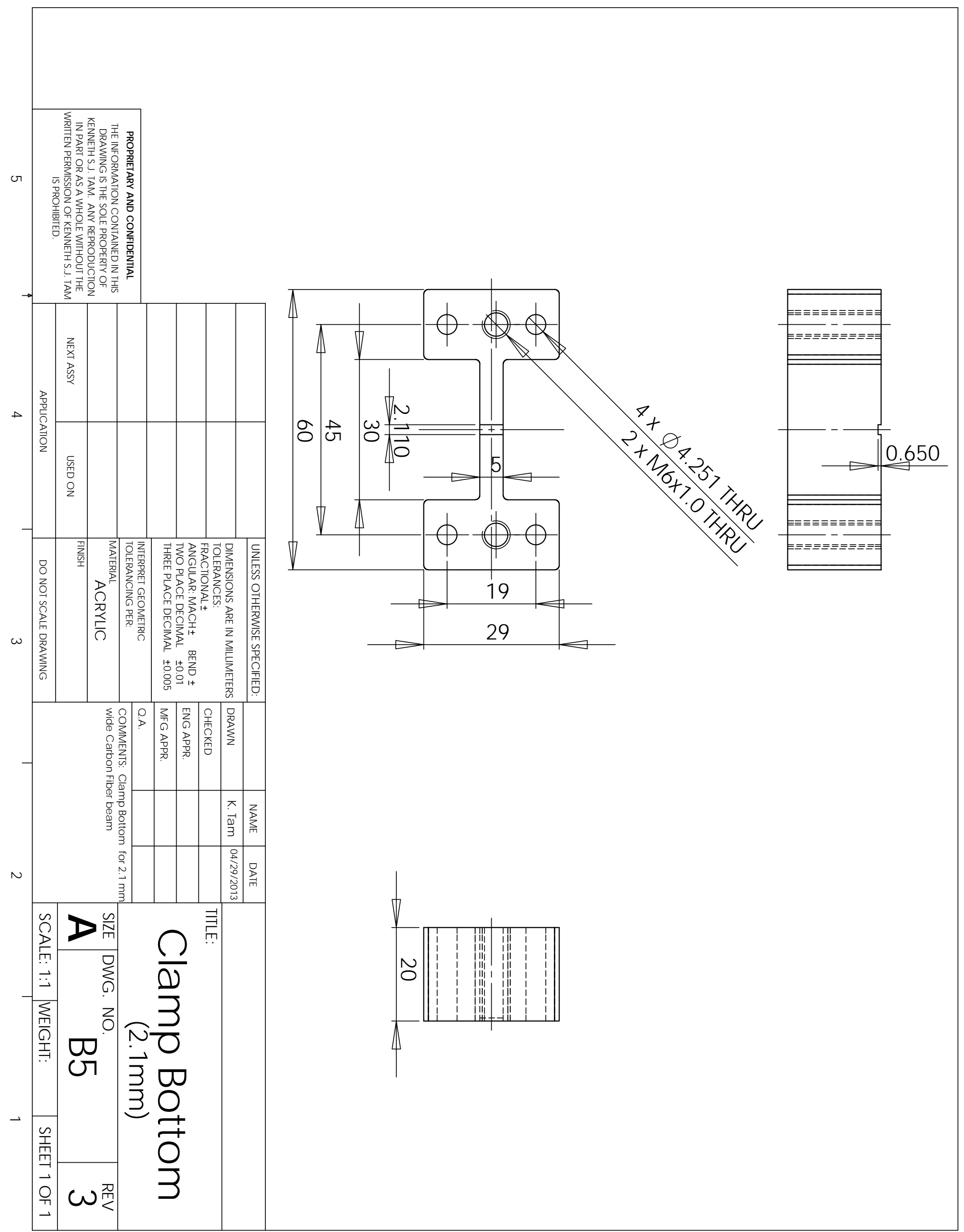




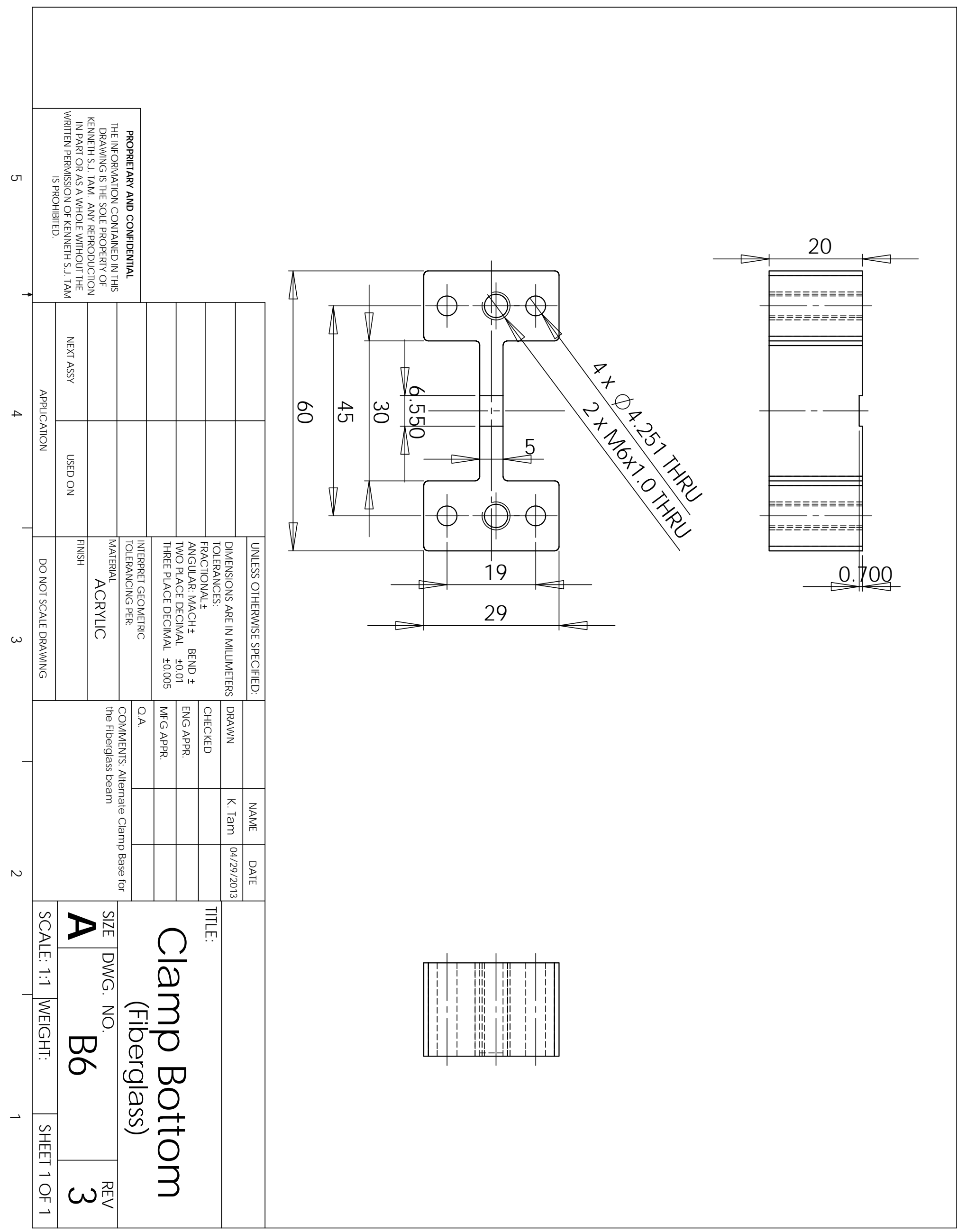




\section{References}

[1] A. Harb, "Energy harvesting: State-of-the-art," Renewable Energy: Generation \& Application, vol. 36, no. 10, pp. 2641-2654, 2011.

[2] S. Roundy, P. K. Wright and J. Rabaey, "A study of low level vibrations as a power source for wireless sensor nodes," Computer Communications, vol. 26, no. 11, p. 1131-1144, 2003.

[3] C. B. Williams and R. B. Yates, "Analysis Of A Micro-electric Generator For Microsystems," in Proceedings of the 8th International Conference on Solid-State Sensors and Actuators Eurosensors IX, Stockholm, June 1995.

[4] P. D. Mitcheson, T. C. Green, E. M. Yeatman and A. S. Holmes, "Architectures for Vibration-Driven Micropower Generators," Journal of Microelectromechanical Systems , vol. 13, no. 3, pp. 429-440, June 2004.

[5] S. P. Beeby, M. J. Tudor and N. M. White, "Energy harvesting vibration sources for microsystems applications," Measurement Science And Technology, vol. 17, no. 12, pp. R175- R197, 6 October 2006.

[6] D. P. Arnold, "Review of Microscale Magnetic Power Generation," IEEE Transcactions on Magnetics, vol. 43, no. 11, pp. 3940- 3951, November 2007.

[7] C. Shearwood and R. Yates, "Development of an electromagnetic microgenerator," Electronics Letters, vol. 33, no. 22, pp. 1883-1884, October 1997.

[8] M. Mizuno and D. G. Chetwynd, "Investigation of a resonance microgenerator," Journal of Micromechanics and Microengineering, vol. 13, no. 2, p. 209-216, 2003.

[9] H. Kulah and K. Najafi, "An Electromagnetic Micro Power Generation for Low-Frequency Environmental Vibration," in 17th IEEE International Conference on. (MEMS), Maastricht, January 2004.

[10] S. P. Beeby, R. N. Tora, M. J. Tudor, P. Glynne-Jones, T. O’Donnell, C. R. Saha and S. Roy, "A micro electromagnetic generator for vibration energy harvesting," Journal of Micromechanics and Microengineering, vol. 17, no. 7, p. 1257-1265, 2007.

[11] S. Scherrer, D. G. Plumlee and A. J. Moll, "Energy Scavenging Device in LTCC Materials," in IEEE Workshop on Microelectronics and Electron Devices, 2005, April 2005.

[12] I. Sari, T. Balkan and H. Kulah, "An electromagnetic micro power generator for wideband environmental vibrations," Sensors and Actuators A, Vols. 145-146, p. 405-413, 2008.

[13] M. S. M. Soliman, E. M. Abdel-Rahman, E. F. El-Saadany and R. R. Mansour, "A wideband vibration-based energy harvester," Journal of Micromechanics and Microengineering, vol. 18, no. 11, pp. 1-11, 2008. 
[14] J. Fraden, Handbook of Modern Sensors - Physics, Designs and Applications, 3rd ed., New York: Springer, 2004.

[15] P. Basset, D. Galayko, A. M. Paracha, F. Marty, A. Dudka and T. Bourouina, "A batchfabricated and electret-free silicon electrostatic vibration energy harvester," Journal of Micromechanics and Microengineering, vol. 19, no. 11, pp. 1-12, 2009.

[16] S. Meninger, J. O. Mur-Miranda, R. Amirtharajah, A. Chandrakasan and J. Lang, "Vibration-to-Electric Energy Conversion," in 1999 International Symposium on Low Power Electronics and Design, San Diego, August 1999.

[17] S. Meninger, J. O. Mur-Miranda, R. Amirtharajah, A. P. Chandrakasan and J. H. Lang, "Vibration-to-Electric Energy Conversion," IEEE Treansactions on Very Large Scale Integration (VLSI) Systems, vol. 9, no. 1, pp. 64-76, 2001.

[18] E. 0. Torres and G. A. Rincon-Mora, "Electrostatic Energy Harvester and Li-Ion Charger Circuit for Micro-Scale Applications," in Proceedings of the 2006 49th Midwest Symposium on Circuits and Systems, San Juan, Aug. 2006.

[19] S. M. Kaplan, Wiley Electrical and Electronics Engineering Dictionary, Hoboken, New Jersy: John Wiley \& Sons, Inc., 2004.

[20] S. Roundy, P. K. Wright and K. S. J. Pister, "Micro-Electrostatic Vibration-To-Electricity Converters," in Proceedings of IMECE'02, New Orleans, Nov. 2002.

[21] G. D. Pasquale and A. Somà, "Investigations on Energy Scavenging Methods Using MEMS Devices," in CAS 2008 Proceedings, Sinaia, Oct. 2008.

[22] G. Despesse, T. Jager, J.-J. Chaillout, J.-M. Léger and S. Basrour, "Design and Fabrication of a New System For Vibration Energy Harvesting," in Research in Microelectronics and Electronics, Lausanne, July 2005.

[23] G. Despesse, J. Chaillout, T. Jager, F. Cardot and A. Hoogerwerf, "Innovative Structure For Mechanical Energy Scavenging," in The 14th International Conference on Solid-State Sensors, Lyons, June 2007.

[24] D. Hoffmann, B. Folkmer and Y. Manoli, "Fabrication, characterization and modelling of electrostatic micro-generators," Journal of Micromechanics and Microengineering, vol. 19, no. 9, pp. 1-11, 2009.

[25] B. Yang, C. Lee, R. K. Kotlanka, J. Xie and S. P. Lim, "A MEMS rotary comb mechanism for harvesting the kinetic energy of planar vibrations," Journal of Micromechanics and Microengineering, vol. 20, no. 6, pp. 1-11, 2010.

[26] H. A. Sodano, D. J. Inman and G. Park, "A Review of Power Harvesting from Vibration Using Piezoelectric Materials," The Shock and Vibration Digest, vol. 36, no. 3, pp. 197-205, 
2004.

[27] Y. Jeon, R. Sood, L. Steyn and S.-G. Kim, "Energy Harvesting MEMS Devices Based on d33 Mode Piezoelectric Pb(Zr,Ti)O3 Thin Film Cantilever," in CIRP Seminar on Micro and Nano Technology, Copenhagen, Nov. 2003.

[28] R. Sood, Y. Jeon, J.-h. Jeong and S. Kim, "Piezoelectric micro power generator for energy harvesting," in Proc. of Solid-State Sensor and Actuator Workshop, Hilton Head, June 2004.

[29] F. Lu, H. P. Lee and S. P. Lim, "Modeling and analysis of micro piezoelectric power generators for micro-electromechanical-systems applications," Smart Materials and Structures, vol. 13, no. 1, pp. 57-63, 2004.

[30] R. Sood, Y. Jeon, J.-h. Jeong and S. Kim, "Piezoelectric micro power generator for energy harvesting," Proc. of Solid-State Sensor and Actuators, 2004.

[31] Y. Jeon, R. Sood, J.-h. Jeong and S.-G. Kim, "MEMS power generator with transverse mode thin film PZT," Sensors and Actuators A, vol. 122, no. 1, p. 16-22, 2005.

[32] Y. Ammar, A. Buhrig, M. Marzencki, B. Charlot, S. Basrour, K. Matou and M. Renaudin, "Wireless sensor network node with asynchronous architecture and vibration harvesting micro power generator," in Proc. 2005 Joint Conf. on Smart Objects and Ambient ibeuntelligence: Innovative Context-Aware Services: Usages and Technologies, Grenoble, October 2005.

[33] S. Roundy and Y. Zhang, "Toward self-tuning adaptive vibration based micro-generators," in Proc. SPIE 5649, Smart Structures, Devices, and Systems II, Sydney, Dec. 2004.

[34] H.-B. Fang, J.-Q. Liu, Z.-Y. Xu, L. Dong, L. Wang and D. Chen, "Fabrication and performance of MEMS-based piezoelectric power generator for vibration energy harvesting," Microelectronics Journal, vol. 37, no. 11, p. 1280-1284, 2006.

[35] H.-B. Fang, J.-Q. Liu, Z.-Y. Xu, L. Dong, D. Chen, B.-C. Cai and Y. Liu, "A MEMS-based piezoelectric power generator for low frequency vibration energy harvesting," Chinese Physics Letters, vol. 23, no. 3, pp. 732-734, 2006.

[36] V. R. Challa, M. G. Prasad, Y. Shi and F. T. Fisher, "A vibration energy harvesting device with bidirectional resonance frequency tunability," Smart Materials and Structures, vol. 17, no. 1, pp. 1-10, 2008.

[37] D. Shen, J.-H. Park, J. Ajitsaria, S.-Y. Choe, H. C. Wikle and D.-J. Kim, "The design, fabrication and evaluation of a MEMS PZT cantilever with an integrated Si proof mass for vibration energy harvesting," Journal of Micromechanics and Microengineering, vol. 18, no. 5, pp. 1-7, 2008.

[38] P. Muralta, M. Marzencki, B. Belgacem, F. Calame and S. Basrour, "Vibration Energy Harvesting with PZT Micro Device," in Proceedings of the Eurosensors XXIII conference, 
Lausanne, Sept. 2009.

[39] B. S. Lee, S. C. Lin, W. J. Wu, X. Y. Wang, P. Z. Chang and C. K. Lee, "Piezoelectric MEMS generators fabricated with an aerosol deposition PZT thin film," Journal of Micromechanics and Microengineering, vol. 19, no. 6, pp. 1-8, 2009.

[40] Y. Tadesse, S. Zhang and S. Priya, "Multimodal Energy Harvesting System: Piezoelectric and Electromagnetic," Journal of Intelligent Material Systems and Structures, vol. 20, no. 5, pp. 625-632, 2009.

[41] C. Eichhorn, F. Goldschmidtboeing and P. Woias, "Bidirectional frequency tuning of a piezoelectric energy converter based on a cantilever beam," Journal of Micromechanics and Microengineering, vol. 19, no. 9, pp. 1-6, 2009.

[42] B. S. Lee, S. C. Lin and W. J. Wu, "Fabrication and Evaluation of a MEMS Piezoelectric Bimorph Generator for Vibration Energy Harvesting," Journal of Mechanics, vol. 26, no. 4, pp. 493-499, 2010.

[43] L.-C. J. Blystad, E. Halvorsen and S. Husa, "Piezoelectric MEMS Energy Harvesting Systems Driven by Harmonic and Random Vibration," IEEE Transactions on Ultrasonics, Ferroelectrics, and Frequency Control, vol. 57, no. 4, pp. 908-919, 2010.

[44] C. Q. C. J. T. T. K. ,. C. L. Huicong Liu, "A MEMS-based piezoelectric cantilever patterned with PZT thin film array for harvesting energy from low frequency vibrations," in 2011 International Conference on Optics in Precision Engineering and Nanotechnology, Signapore, March 2011.

[45] H. Liu, C. Lee, T. Kobayashi, C. J. Tay and C. Quan, "A MEMS-based wideband piezoelectric energy harvester system using mechanical stoppers," in Electron Devices Meeting (IEDM), 2011 IEEE International, Washington, DC, Dec. 2011.

[46] A. Hajati and S.-G. Kim, "Ultra-wide bandwidth piezoelectric energy harvesting," Applied Physics Letters, vol. 99, no. 8, p. 105, 2011.

[47] C. Eichhorn, R. Tchagsim, N. Wilhelm, G. Biancuzzi and P. Woias, "An energyautonomous self-tunable piezoelectric vibration energy harvesting system," in 2011 IEEE 24th International Conference on Micro Electro Mechanical Systems (MEMS), Cancún, Jan. 2011.

[48] A. Abdelkef, F. Najar, A. H. Nayfeh and S. B. Ayed, "An energy harvester using piezoelectric cantilever beams undergoing coupled bending-torsion vibrations," Smart Materials and Structures, vol. 20, no. 11, pp. 1-11, 2011.

[49] W. Al-Ashtari, M. Hunstig, T. Hemsel and W. Sextro, "Frequency tuning of piezoelectric energy," Smart Materials and Structures, vol. 21, no. 3, pp. 1-8, 2011. 
[50] A. Bokian, "Natural frequencies of beams undercompressive axial loads," Journal of Sound and Vibration, vol. 1, no. 126, pp. 49-65, 1988.

[51] F. J. Shaker, "Effect of Axial Load on Mode Shape and Frequencies of Beams," National Aeronautics and Space Administration, Washington D. C., 1975.

[52] Johnson Matthey, "Data Sheet Piezoceramic Trimorph Bending Actuator," Johnson Matthey, Redwitz, 2011.

[53] E. A. Avallone, T. Baumeister and A. Sadegh, Marks' Standard Handbook for Mechanical Engineers, 11th ed., New York: McGraw Hill, 2007.

[54] W. H. Press, S. A. Teukolsky, W. T. Vetterling and B. P. Flannery, Numerical Recipes in C, 2nd ed., New York: Cambridge University Press, 1992.

[55] M. H. Hayes, Statistical Digital Signal Processing and Modeling, Hoboken, New Jersey: John Wiley \& Sons, Inc., 1996, pp. 442-443.

[56] E. Bruce, Biomedical Signal Processing and Signal Modeling, Toronto: John Wiley and Sons, 2001, p. 377.

[57] The MathWorks, Inc., "MATLAB Help Files," Natick, 2010.

[58] ANSYS Inc., Help System, ANSYS Inc..

[59] R. L. Mason, R. F. Gunst and J. L. Hess, Statistical Design and Analysis of Experiments With Applications to Engineering and Science, Hoboken, New Jersey: John Wiley \& Sons, 2003.

[60] R. Reed, S. Oh and R. J. Marks, "Regularization Using Jittered Training Data," in International Joint Conference on Neural Networks, Baltimore, 1992.

[61] T. Masters, Practical Neural Network Recipes in C++, New York: Morgan Kaufmann, 1993.

[62] C. Eichhorn, R. Tchagsim, N. Wilhelm and P. Woias, "A smart and self-sufficient frequency tunable vibration energy harvester," Journal of Micromechanics and Microengineering, vol. 21, no. 10, pp. 1-11, 2011. 